\title{
ENYIRONMENTAL LEVELS OF RADIOACTIVITY IN THE VICINITY OF THE LAWRENCE LIVERMORE LABORATORY 1973 ANNUAL REPORT
}

\author{
W. J. Silver, C. I. Lindeken, J. W. Meadows, \\ W. H. Hutcrin, and D. R. Mcintyrs
}

March 4, 1974

Prepared for U.S. Atomic Energy Commission under contract No. W-7405-Eng-48

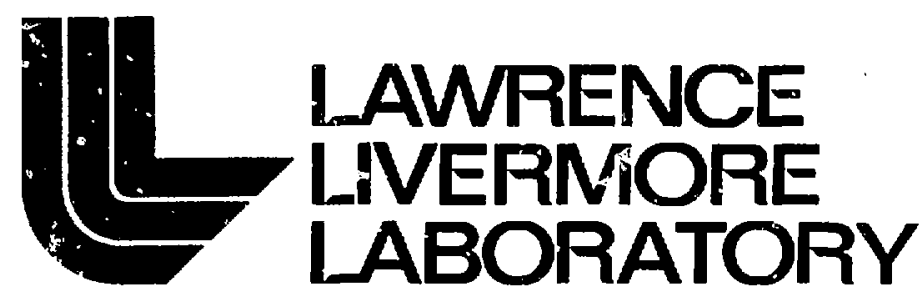

University of Califomia/Livermore 
Notrcl

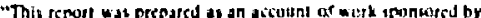
the Uniked Sister fimetnment. Neither the United States not

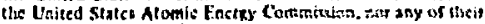
employers, nor any of theit contracton, whashledsturs, of listip

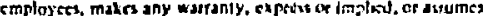

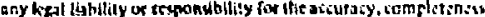

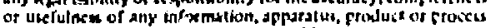

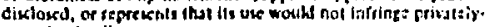
uwned rthit."

Printed in the United States of America Available from

National Technical Information Service

U.S. Departrnent of Commerce 5285 Por Royal Road

Springfield, Virginia 22151

Price: Printed Copy $\$$ ; Microfiche $\$ 0.95$

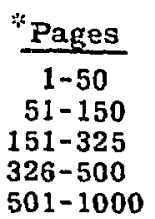

NTIS

Selling Price

$\$ 4.00$

$\$ 5.45$

$\$ 7.60$

$\$ 10.60$

$\$ 13.60$ 


\title{
L4 \\ LAWPENCE UNERMORE LABCAATOFY

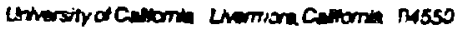

UCR1-51547

\section{ENVIRONAENTAL LEVELS OF RADIOACTIVITY IN THE VICINITY OF THE \\ LAWRENCE LIVERMORE LABORATTORY 1973 ANNUAL REPORT}

\author{
W. J. Silver, C. L. Lindeken, J. W. Meadows, \\ W. H. Hutchin, and D. R. Mintyre
}

MS. date: March 4, 107t

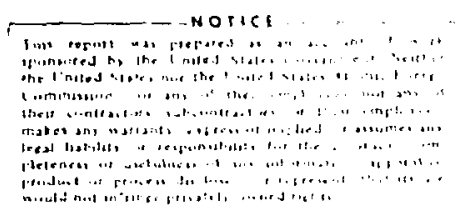




\section{Foreword}

This report is prepared for the I.S. Atomic Hergy Commission by the Envitonmental Evaluations (iroup of the Hatards Corirol Department it the Law rence Livermore I aboratory. Dat a are obtaned through the combined affe-ts of the Kadiochemistry bivision, the Bio-Medisal Division and the Hazards Control Department.

\section{Contents}

Abstract

Introduction

Lnvironmental Levels of Radioactivity - Livermore Laboratory

Atmospheric Fia.iioacisvity

Enviroumental Impact of LLL Airborne Efrluents . . . . . . . is

Soil . . . . . . . . . . . . . . . . . . . . . . 8

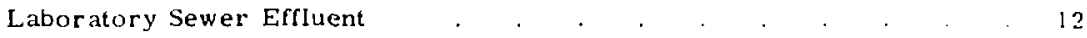

Environrental Impact of LLL Liquid Effluents . . . . . . 12

Water . . . . . . . . . . . . . . . . . . . . . 12

Vegetation . . . . . . . . . . . . . . . . . . 4.3

Envizonmental Ridiation Measurements . . . . . . . . . . Ij

Environmental Levels of Radioactivity - Site 300 . . . . . . . . 16

Atmospheric Radioactivity . . . . . . . . . . . . . $\quad 16$

Ecology Impact Study . . . . . . . . . . . . . . . . . 16

Soil . . . . . . . . . . . . . . . . . . 18

Water . . . . . . . . . . . . . . . . . . . . . 19

Vegetation . . . . . . . . . . . . . . . . . . . . . . . . . . . . 20

Milk . . . . . . . . . . . . . . . . . . . 21

References . . . . . . . . . . . . . . . . . . . . . 47

Appendix - Environmental Activity Guide Levels . . . . . . . . . 44 


\title{
ENVIRONMENTAL LEVELS OF RADIOACTIVITY IN THE VICINITY OF THE LA WRENCE LIVERMORE LABORATORY 1973 ANNÜAL REPORT
}

\begin{abstract}
The: Lawrence livermore Laboratory continuously monitors the levels of radioactivity within the Livermore Valley and Site 300. Results ff inalyser performed during 1973 for gross radioactivity and for specific radiunuclides of ir terest in a variety of environmental samples are presented in this repurt. In all cases, the levels of activity observed during 1973 were found to be below the appropriate concentration guide values in AEC Manual Chapter 0524.

Particulace air filters showed gross beta activities lower than those observed during 1972 , reflecting a reduction in glabal fallout in the atmosphere. Gamina spectral measurements on Laboratory perimeter al: filters also showed lower activities of global fallout gamma emit ters. These perimeter air samples were analyzed for ${ }^{239} \mathrm{Pu},{ }^{238} \mathrm{Pu},{ }^{90} \mathrm{Sr}, 235 \mathrm{~L}$. and ${ }^{238} \mathrm{U}$. With the exception of one sampling location, the annual average ${ }^{239} \mathrm{Pu}$ concentration was $1.3 \times 10^{-17} \mu \mathrm{Ci} /$ mi, typical of global fallout.

Air simples taken within Site 300 were analyzed for turanium. These analyses showed a lower than normal ratio of $235_{\mathrm{U} /}{ }^{238}$ U. This is due to "depleted" uraniun (uranium which is specially processed to lower the $225 \mathrm{U}$ content) used at the Site. Airburne uranium concentra-

tions were well below the standards set by the AEC.

Soll samples collected in the off-site vicinity of LLL perimeter boundaries and at Site 300 were analyzed for plutonium, uranium, and gamma emitting radionuclides. Traces of flutontum rbove global background levels were detected in two off-site samples near the east perimeter of the Laboratory. Sediment samples collected in surface drainage pathways from LLL showed that the plutonium in these sediments was in the same runge observed in soil samples collected in the Livermore Valley.

Site 300 soil samples indicate depleted levels of ${ }^{235} \mathrm{U}$ near firing bunkers, but ${ }^{235} \mathrm{U} /{ }^{238} \mathrm{U}$ ratios appruach that of natural uranium at site perimeters. These data indicate no apparent change from that observed in 1972 .

Water samples collected within the Livermore Valley exhibited normal background gross beta and tritium activities.

Gamma spectral analyses of vegetation sumples revealed no gamma-emitting radionuclides other than those present naturally or in global fallout. The vegetation samples collected in areas generally downwind from the Livermore Laboratory revealed tritium activities 10 to 100 times higher than those collected in are 35 where
\end{abstract}


the Laboratory's contribution should be minimal. However, if tho vegetation were a regular part of one's diet, the annual whole body radiation dose fror, tritium would be less than 1 mrem.

The off-site radiation exposure rates measured by thermoluminescent dosimeters were in the range to be expected from the naturally occurring radionuclides in the soil and feom local cosmic radiation.

Assessment of the racliation dosest to an individual from the observed environ. mental activitues listed an this report indicatee the contribution from artuficially produced radionuclides is sis.all in comparison with the uppreximately 100 mrem yr dose received from natural sources.

\section{Introduction}

The Lawrence Livermor: 1, aboratory is located about 50 miles southeast of San Francisco in the I ivermore Valley. Shielded from the ocean by the wesiern hills, the valley has a warm, dry climate. Annual rainfall is about $14 \mathrm{in}$. and occurs primarily during the winter months in connection with $P$ acific storms. Surface water arainage from the valley is from east to west through various arroyos, with outflow new Sund in the southwestern corner of the valley. I'revailing winds are from the west and soutluwest during April through September. During the remainder of the jear, the winds rrom the east and nortieast occur almost as frequently as those from the west and southwest.

The Livermore I.aboratory occuples an area of one sq $\mathrm{mi}$ and is situated approximately three mi east of Livermorc. The Laboratory plays an integral part in the nation's nuclear weupons development program and makes diversified researches into controlled thermonuclear reactions, industrial applications of nuclear explosives, and the effects of radiation on the biosphere.

Much of the materials testing and highexplosive diagnostic work of the Laboratory is carried on at site 300, . 10-sq $\mathrm{mi}-m$ located about $13 \mathrm{mi}$ southuast of liveraoro in the sparsely populated hills o! the Diablo Range which separates the Livermore and San Joakuin Valleys.

In order to carry out these programa, the Laboratory handles a variety of potentially hazardous rarjio ictive materials. A strict etauent control profr.tm, which places naximum emphasis on controllinis the effluents at the = urce, has boen in continuous existence since the laboratory began operation. An envionmental surveillance program is conducted to ensure that this effuent control program is indeed restricting the release of radioactivity from the I.ivermore [.aboratory and Situ 300 te concentrations well below the standasds set forth by the IJ. $\mathrm{s}$. Itomic Entrgy Commission. This program employs techniques with sensitivities usually capable of detecting radioucturity below environmental back ground levels. The program includes the collection of aicborne particulates, soil, water, sewer effluent, veretation, and milk samples. These samples are analyzed for gross radicactivities as well ats for the actuvity of specific radionuclides of interest. In 
addition, onvirommontal backeround rabiation is mensared at numerous loca = tions in the vermity of the Livermore laboratory by meths of thermoluminescent detectors.

The results of the analyses are provided in this repo:t. When appropriate, maximum, minimum, asd average concontrations are given, Error limits, when included, roflect the uncertanties in the analyses at the $95 \%$ confidence level due

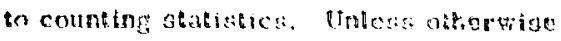
statcet, the limit of tetectrors of thesc

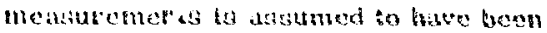
reacher when the a gerror tre toog. An attempt has boen made ta assogs the Impact from the observed envisonfment al actluty loweld of artifichally and naturally produced radionuclided by cistonating the whole body of critical orfian toses delivered to an ardult by tile rartons radion nt:slides if tatorest.

\section{Environmental Levels of Radioactivity - Livermore Laboratory}

\section{ATMOSPHIRLE RAIOACTIVIT}

\section{Concentrations ar various airborne} radionuclides were measured at 17 sampling station: situated throuthout the Livermare valley. Their locittons are shown in lins. 1 and 2 . The alx satmplers located on the l.aboratory perimeter $u: j o$ 80-1n. Whatm.n-11 thters, 'lhe average simpling rate was 25 r.fm. The remalsinge simplers, lncited off-site, used $36-\mathrm{ln}^{2}$ HV - 70 (cellulose-asbestos) fllters and were operated at an average flow rate of $4 \mathrm{cfm}$. Air filters are changed weekly.

Air sianplers are situated in suen a manner that they provide a reasonable assurance that a significant release of airborne particulate radioactivity from the Laboiatory would be detectable regardless of the local meteorologi at the time of the release.

ifter a four day delay for the decay of radon-thoron daughters, gross alpi.d and beta activities on the filters are determined using an automatic gas flow proportional counting system. Monthly composites of the perimeter filters are

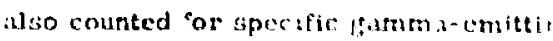
radionuclides by means of a Ge(L., tetector equipped with a Curnton suppes sion bystem. Follewtur ramma countm: filters are : subdividu! into monthly com possite: by samplint Incation tite. 3 .

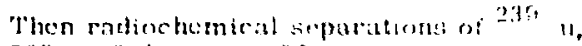
$23 \mathrm{Pu}^{2}{ }^{24} \mathrm{I}_{\mathrm{Am}}$, and ${ }^{0} \mathrm{sr}$ ate made, , , topic ubantum analysas a pereform, by mass spect:"umetry on portions of these simples.

The feross hetis actutiticss (averaged over six month fretods) and the annual average activitles are lasted for each sample locintion in Table 1, where they may be compured witt the approprate Radionetivity Concentration Guide (RCG) of the AFC Manual Chister 0524. In addition, the weoli'y averuge gross beta actlvities on the alr filters from $: 11$ Livermore Valley staions are shown in Fig. 3.

We have observed a signific ant decrease in the level of gross beta activity in Livermore air over the past three years. in 1971 the averar? was $1.1 \times 10^{-13} \mu \mathrm{Ci}$ $\mathrm{ml}^{1}$ in 1.972 the average was $7.3 \times 10^{-14}$ 


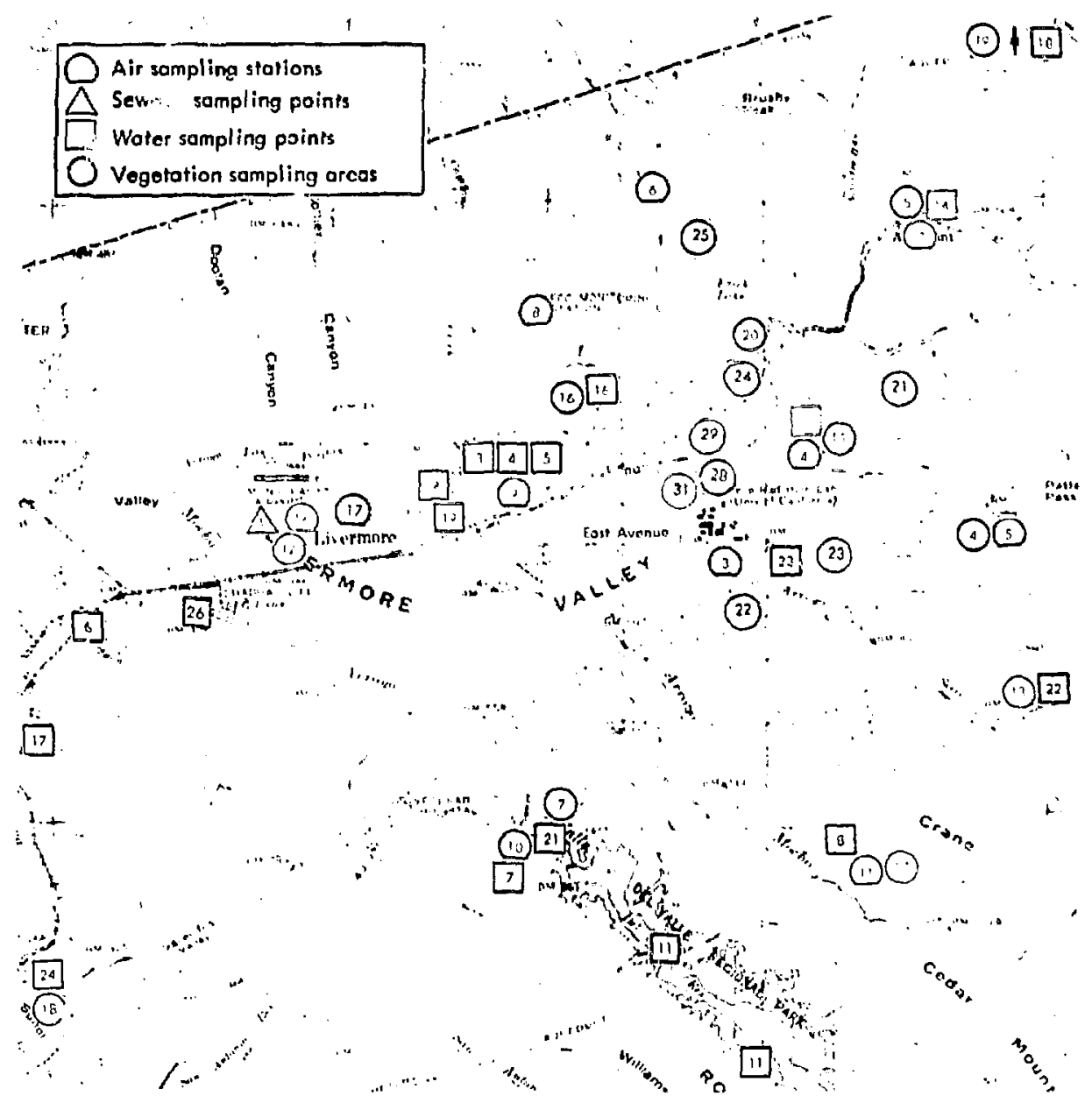

Fig. 1. Lawrence Livermore Laboratory off-site environmental sampling locations.

(Ref. 2), and in 1973 the average dropped to $2.1 \times 10^{-14}$. The gross beta activity is due to global fallout from nuclear weapons tests, to radionuclides such as ${ }^{7} \mathrm{~B} \in$ produced by cosmic -ray interactions with the atmosphere, and to naturally occurring radionuclides. This is shown in Table 2, which lists the activities of the more abundant gamma-emitting radionuclides in monthly composite samples collected by the six Laboratory perimeter samplers. Neither these data nor the beta activity exhibit the typical spring increase in surface air radioactivity. 


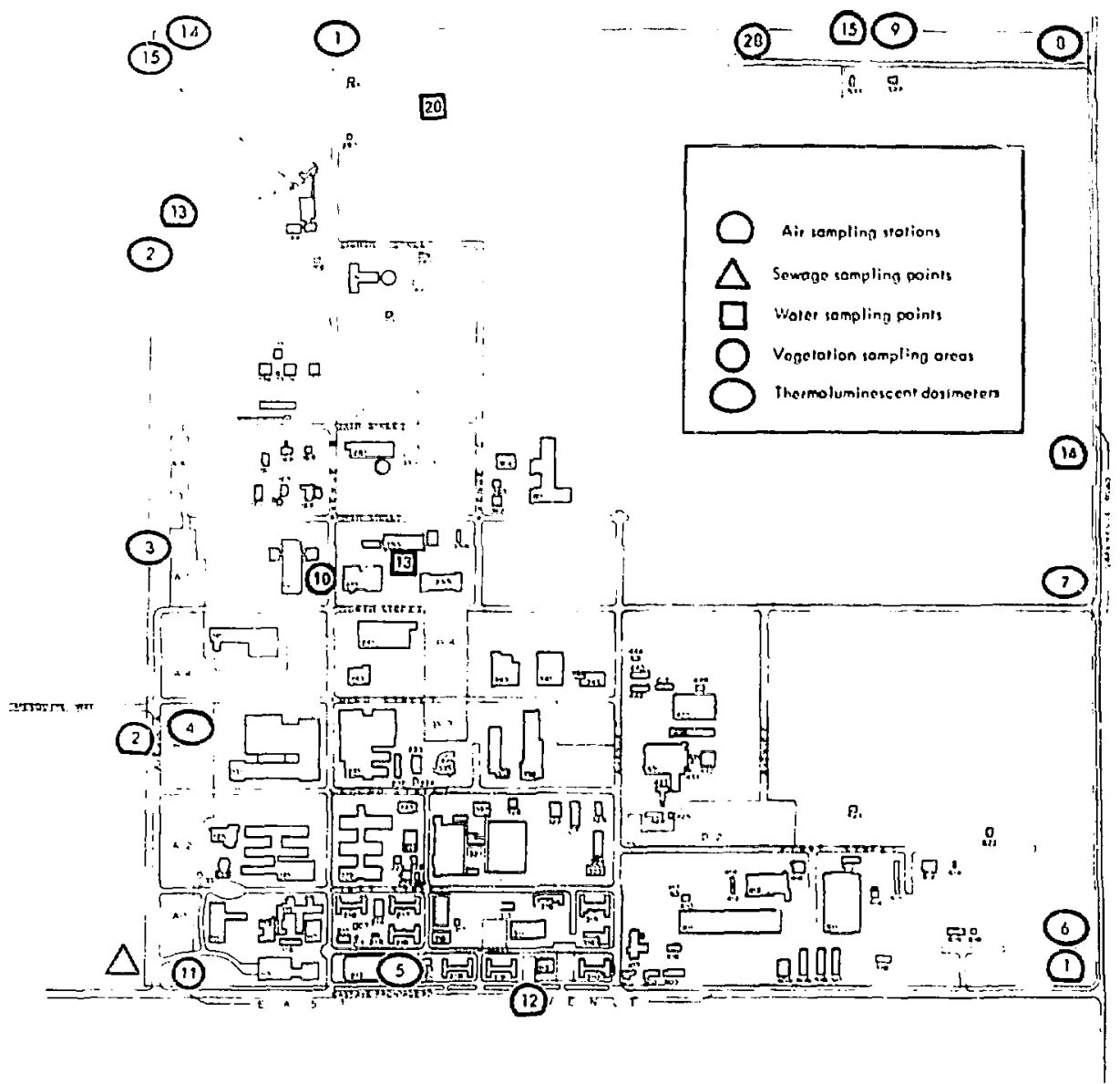

Fig. 2. Lawrence Llvermore Laboratory on-site environmental sampling locations.

Table 3 shows the concentrations of airborne ${ }^{239} \mathrm{Pu},{ }^{238} \mathrm{Pu},{ }^{241} \mathrm{Am}$, and ${ }^{90} \mathrm{Sr}$ based on the analysis of LLL perimeter air filters. With the exception of Location 14 on the east perimeter, which is norma!ly downwind from Laboratory operations, all activities are typical of global fallout. The ${ }^{239} \mathrm{Pu}$ air concentra- tion observed at Location 14 during April may have occurred during transfer of dry sludge from one of the sclar evaporators used in volume reduction of certain low level liquid waste. These evaporators are located southwest (normally upwindsee wind rose in Fig. 4) of this sampling location. Upon recognition of this 


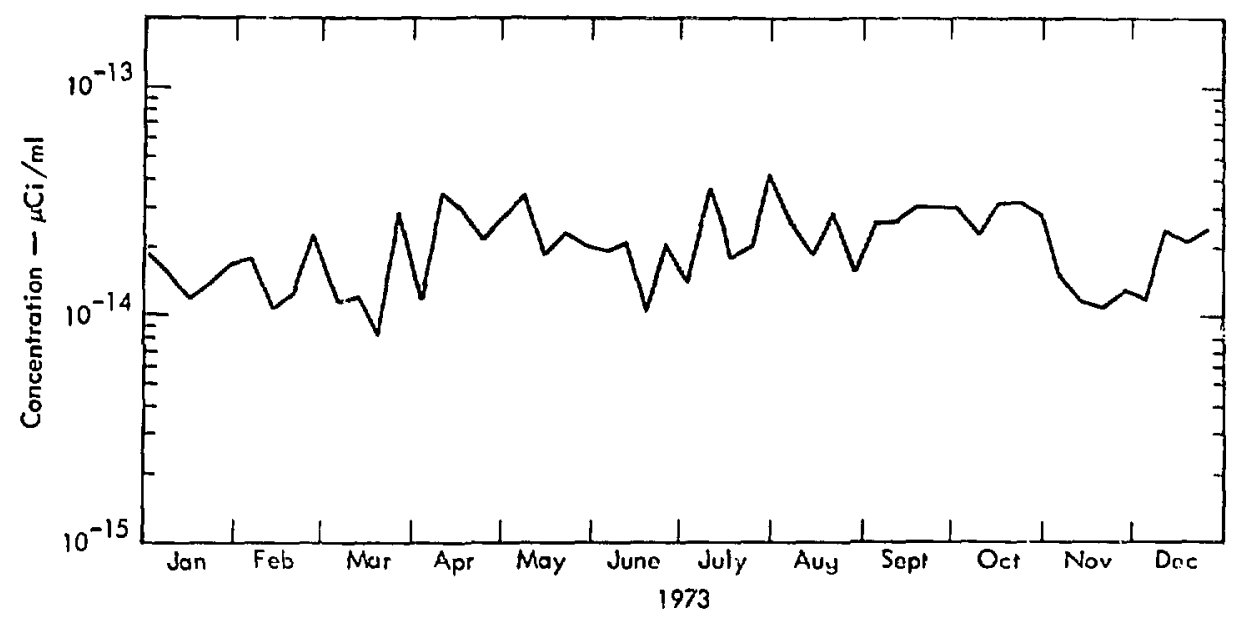

Fig. 3. Weekly average gross beta activity on alr filters from Livermore Valley siumpling loc ations,

possibility, operations were changed to minimize contact of the sludge with air.

Measurements of tritiated water (HTO) in air at LLL perimeter air sampling locations were begun in 1973. Water vapor was collected in silica gel samplers operated at about 0.5 liter per minute for one week periods. Flow control was maintained by an in-line critical orifice. The water collected was recovered by freeze drying and the HTO was measured by liquid scintillation counting. Table 4 shows the concentrations by location (see Fig. 2).

ENVIRONMENTAL IMPACT OF LLL AIRBORNE EF:-LUENTS

The total curie quantities of airborne radioactivity released by Laboratory operations during 1973 are listed in this section. These data are included so that we can compare the quantities of specific radionuclides relcased $w$ ith their apparent impact on the environment based on relevant monitoring data.

\section{41 Argon}

An estimated total of 1300 curies of 41 Ar was released from the Laboratory's 3 MW pool type reacter. This led to an estimated maximum site-boundary dose of 17 mrem per year. From stack monitoring data at the source and the use of a meteorological diffusion model, ${ }^{3}$ the following estimated $m$ an rem radiation doses were obtained within a radius of 50 miles of L.LL:

During wet season (Noyember

$$
\text { through April) }=2.52 \mathrm{man} \mathrm{rem}
$$

During $\mathrm{dr}: 3$ season (May through

October) $=.35$ man rem total annual $=3.88 \mathrm{man}$ rem 


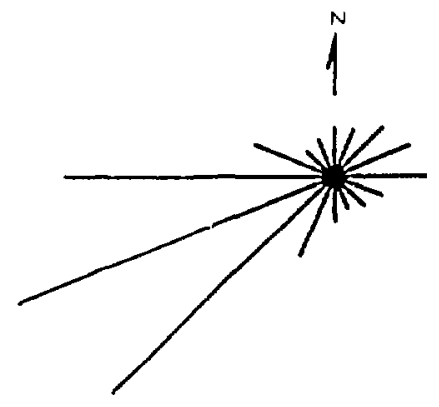

Wind rote thow relativo feequency of wind ditection (by the length of the line) obroinod from the tabulated annual dato below

Frequency of Wind In Poreont for Livarmoro - 1971

\begin{tabular}{|c|c|c|c|c|c|c|c|c|c|c|c|c|c|c|c|c|c|}
\hline $\mathrm{Mn}_{\mathrm{n}}$ & $N$ & NINE & NE & ENE & $\mathbf{E}$ & ESE & SE & SSE & $s$ & SSW & sw & wsw & $w$ & WNW & $N W$ & NNW & Collm \\
\hline Jan & 2.7 & 2.2 & 72 & 12.1 & 17.8 & 6.8 & 6.2 & 2.2 & 3.9 & 3.8 & 4.9 & 7.0 & 9.7 & 7.6 & 2.0 & 2.0 & 1.3 \\
\hline Feb & 2.2 & 2.5 & 6.9 & 12.9 & 14.8 & 7.2 & 5.1 & 2.8 & 3.5 & 3.0 & 4.9 & 8.0 & 11.2 & 0.5 & 3.4 & 2.0 & 1.6 \\
\hline Mar & 2.1 & 2.2 & 6.4 & 12.3 & 13.5 & 6.9 & 4.9 & 2.7 & 3.3 & 2.0 & 4.6 & 10.5 & 12.2 & 9.1 & 3.1 & 1.8 & 1.7 \\
\hline April & 2.1 & 2.3 & 4.6 & 8.7 & 0.4 & 5.1 & 3.2 & 2.1 & 3.0 & 4.8 & 10.8 & 10.1 & 13.9 & 7.4 & 2.2 & 1.8 & 2.3 \\
\hline May & 2.2 & 1.9 & 3.8 & 0.5 & 7.1 & 4.0 & 2.4 & 1.7 & 2.4 & 4.7 & 15.3 & 17.7 & $: 6.7$ & 6.0 & 1.9 & 1.9 & 3.8 \\
\hline June & 2.1 & 1.7 & 3.4 & 5.6 & 0.2 & 3.5 & 2.1 & 1.4 & $2 . \ddot{z}$ & 4.6 & 16.9 & 19.8 & 17.7 & 5.4 & 1.7 & 1.6 & 3.9 \\
\hline July & 1.7 & 1.4 & 2.8 & 4.6 & 5.1 & 2.9 & 1.8 & 1.0 & 2.0 & 5.3 & 19.5 & 22.9 & 17.2 & 4.6 & 14 & 1.4 & 4.0 \\
\hline Aug & 1.6 & 1.4 & 2.7 & 4.5 & 5.0 & 2.8 & 1.7 & 1.2 & 2.1 & 5.3 & 18.0 & 23.5 & 17.0 & 4.5 & 1.4 & 1.4 & 4.1 \\
\hline Sepl & 1.9 & 1.4 & 2.5 & 4.0 & 4.4 & 2.5 & 1.6 & 1.3 & 2.2 & 5.4 & 21.1 & 23.3 & 17.3 & 4.7 & 1.4 & 1.2 & 3.7 \\
\hline Oct & 2.4 & 1.0 & 2.7 & $A .1$ & 4.5 & 2.7 & 1.0 & 1.3 & 2.2 & 5.7 & 20.6 & $2 i .5$ & 16.3 & 4.6 & 1.5 & 2.2 & 3.5 \\
\hline Nov & 2.6 & 2.5 & 3.5 & 4.5 & 4.8 & 2.7 & 1.9 & 1.9 & 2.6 & 5.4 & 19.2 & 20.5 & 15.5 & 4.7 & 2.0 & 2.3 & 3.3 \\
\hline & 2.8 & 3.0 & 3.9 & 4.6 & 5.3 & 3.0 & 2.6 & 2.2 & 2.7 & 5.1 & 17.7 & 19.2 & 15.3 & 4.8 & 2.1 & 2.4 & 3.1 \\
\hline $\begin{array}{l}\text { nnuol } \\
\text { verage }\end{array}$ & 2.2 & 2.0 & 4.2 & 7.0 & 8.2 & 4.2 & 2.9 & 1.8 & 2.7 & 4.7 & 14.6 & 17.8 & 15.0 & 6.0 & 2.0 & 1.9 & 3.0 \\
\hline
\end{tabular}

Fig. 4. Typical annual average wind pattern for Livermore, California (LLL data1971).

The estimated population within a 50 mile radius of LLL is $4.6 \times 10^{6}$. Therefore, the estimated average radiation dose per person is $3.9 / 4.6 \times 10^{6}$ $=8.5 \times 10^{-7} \mathrm{rem} / \mathrm{yr}\left(8.5 \times 10^{-4} \mathrm{mrem}\right.$ per year). This radiation dose may be compared with the approximately $100 \mathrm{mrem}$ per person annual radiation dose from naturally occurring radioactivity.

\section{Tritium}

The total tritium released to the atmosphere by the Laboratory from all sources is estimated to be 2510 curies. About $85 \%$ of this tritium is released at the Gaseous Chemistry Building. Asauming the entire activity were released at this location at a constant rate over the entire year, application of the appropriate $X / Q$ values from Ref. 3 shows the concentration at the Site-boundary to be 1.6 $\times 10^{-10} \mu \mathrm{Ci} / \mathrm{ml}$.

The RCG for tritium (HTO) in uncontrolled areas is $2 \times 10^{-7} \mu \mathrm{Ci} / \mathrm{ml}$. Environmental monitoring for HTO at the Site-boundary showe the maximum 
concentration to be $1.8 \times 10^{-10} \mu \mathrm{Cu} / \mathrm{ml}$. The higher concentration predicted by the diffusion model is conservative, since all the tritlum is assumed to be in the form of HTO. The annual whole body radiation lose to an individual at the Site-boundary who might breathe air containing $1.8 \times 10^{-10} \mu \mathrm{Cl} / \mathrm{ml}$ of HTO would be $0.3 \mathrm{mrem}$. This hypothetical radiation dose is small compared to the 100 mrem received from natural radioactivity.

SOIL

Analysis of soil samples collected throughout the Livermore Valley during 1971 and 1972 provided a data base for local distribution of various radionuclides deposited as a result of global fallout as well as from poss ile Laboratory effluents. These data were reported in annual reports for the above periods ${ }^{1,2}$ and serve as $a$ basis for comparing current levels of activity with those observed in the future.

In 1973, several of the earlier locations were resampled as part of a continuing surveillance program and additional samples were collected in the vicinity of LLL Site-tuundaries. A group of samples was also collected in the San Joaquin Valley, since atmospheric diffusion studies show possible air movement eastward from Livermore through the Altamont Pass into this valley. In addition, sediment samples were collected along surface drainage pathways from LLL to determine if there were any releases from the Laboratory via surface drainage.

In previous years, man-made activities observed in local soil were expressed in total deposition urits $\left(\mu \mathrm{Ci} / \mathrm{m}^{2}\right.$ or $\mathrm{mCi} / \mathrm{km}^{2}$ ) so that the data could be com- pored with the datia of 11 ardy and lirey. Soil cores were collected to a depth of 25 centimetert, since it is inpliced when using deposition units that the simple has been collected to a sufficient depth to account for essenially all the activity. While decp core sampling was still employed during 1973 when deposition data were required for purposes of comparison, greatcr emphasis was placed ort surface sampling to a depth of one centimeter. In the case of plutonium, surface sampling is appropriate, because the sltuation of interest is related to airborne concentrations resulting from possible resuspension of surface activity.

All samples were dried, Eround, and blended. For radiochemical analysis, $100 \mathrm{G}$ aliquots were completely dissolved in acid and the radionuclides were determined using standard techniques. For gamma spectra analysis, approximately $300 \mathrm{~g}$ aliquots of soil were sealed in $200 \mathrm{~cm}^{3}$, thin-walled aluminum cans and counted in a shiclded Ge(Li) spectrometer. equipped with a Compton suppression system.

Table 5 shows the activity levels of various radionuclides observed in Livermore Valley suil samples cullected during 1973. Locations of these samples are shown iri Fig. 5. Samples 253 through 294 were collected during June 1973. Samnle 254, taken on the east side of Greenville Road, is located nearly due east from the solar evaporators. Tnis specific area was not sampled in prior years. However, the area represented by Sample 319, just. north of Sample 254, has been sampled annually since 1971 . In October of 1972, this area (Sample 223) showed typical global fallout levels of 


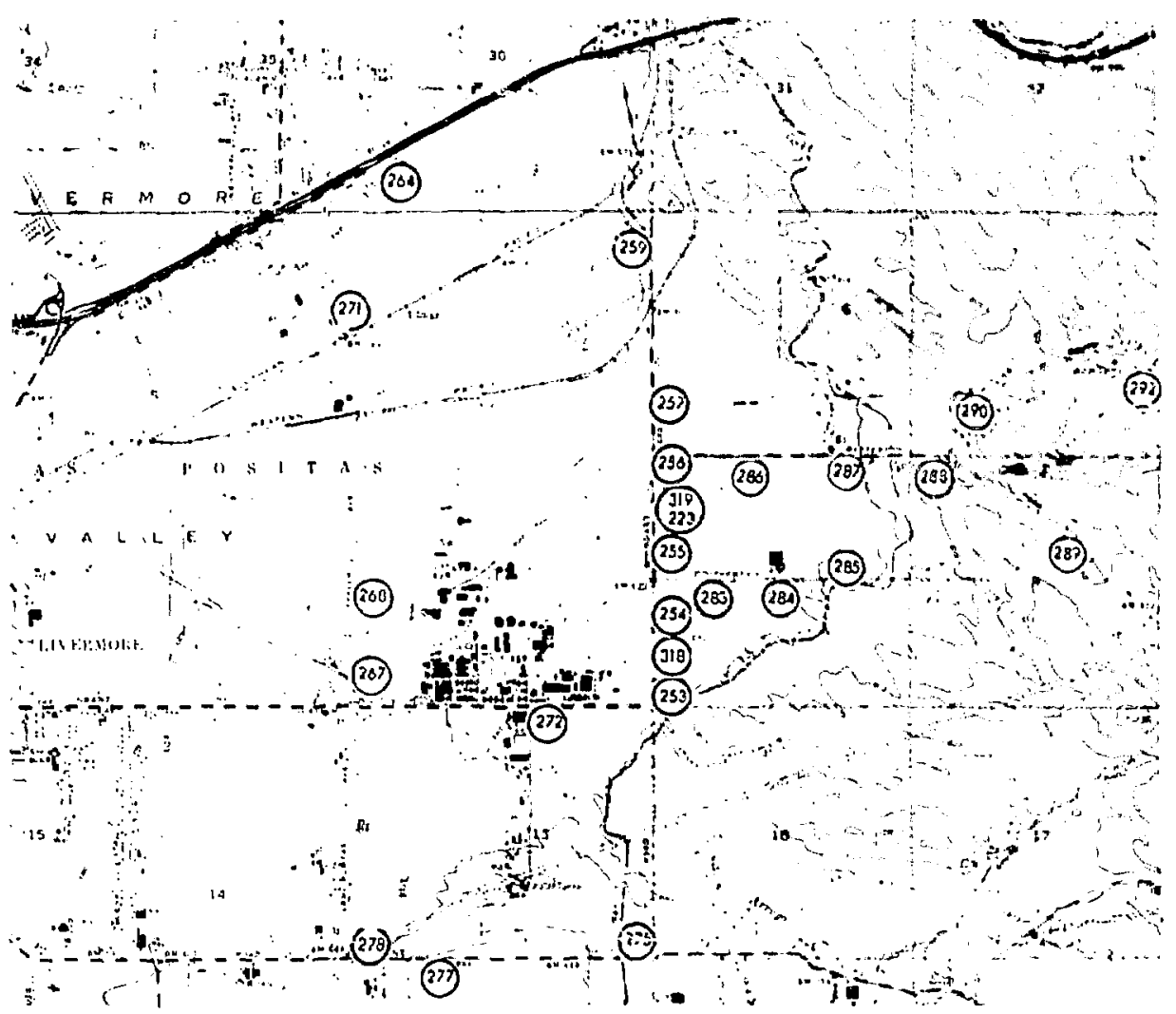

Fig. 5. Locations of soil samples collected in the vicinity of LLL.

plutonium and the small increase observed in $1973+7$ ay possibly be related to operations a he solar evaporators during the sprin of 1973.

Table 6 lists the depusition values of ${ }^{239} \mathrm{Pu},{ }^{238} \mathrm{Pu}$, and ${ }^{137} \mathrm{Cs}$ obtained from the samples coliected in the Jan Joaquin Valley at locations shown in Fig. 6. Median deposition values of ${ }^{239} \mathrm{Pu}$ and ${ }^{137} \mathrm{Cs}$ for $25-\mathrm{cm}$ deep samples were 6.5 $\times 10^{-4}$ and $\cdot .<\times 10^{-2} \mu \mathrm{Ci} / \mathrm{M}^{2}$, respectively. 'These values are typical of those attributed to global fallout in semiarid areas. ${ }^{4}$ The table also shows the results of ${ }^{235} \mathrm{U}$ and ${ }^{238} \mathrm{U}$ analysis performed on these samples using mass spectrometry. In many cases the uranium levels are elevated over that expected for soils in this area of California. 5 Site 300, in the Diablo Range west of the San Joaquin Valley, uses uranium in high explosive experiments. This uranium is "depleted" in ${ }^{235} \mathrm{U}$. Since the ${ }^{235} \mathrm{U} /{ }^{238} \mathrm{U}$ ratios in 


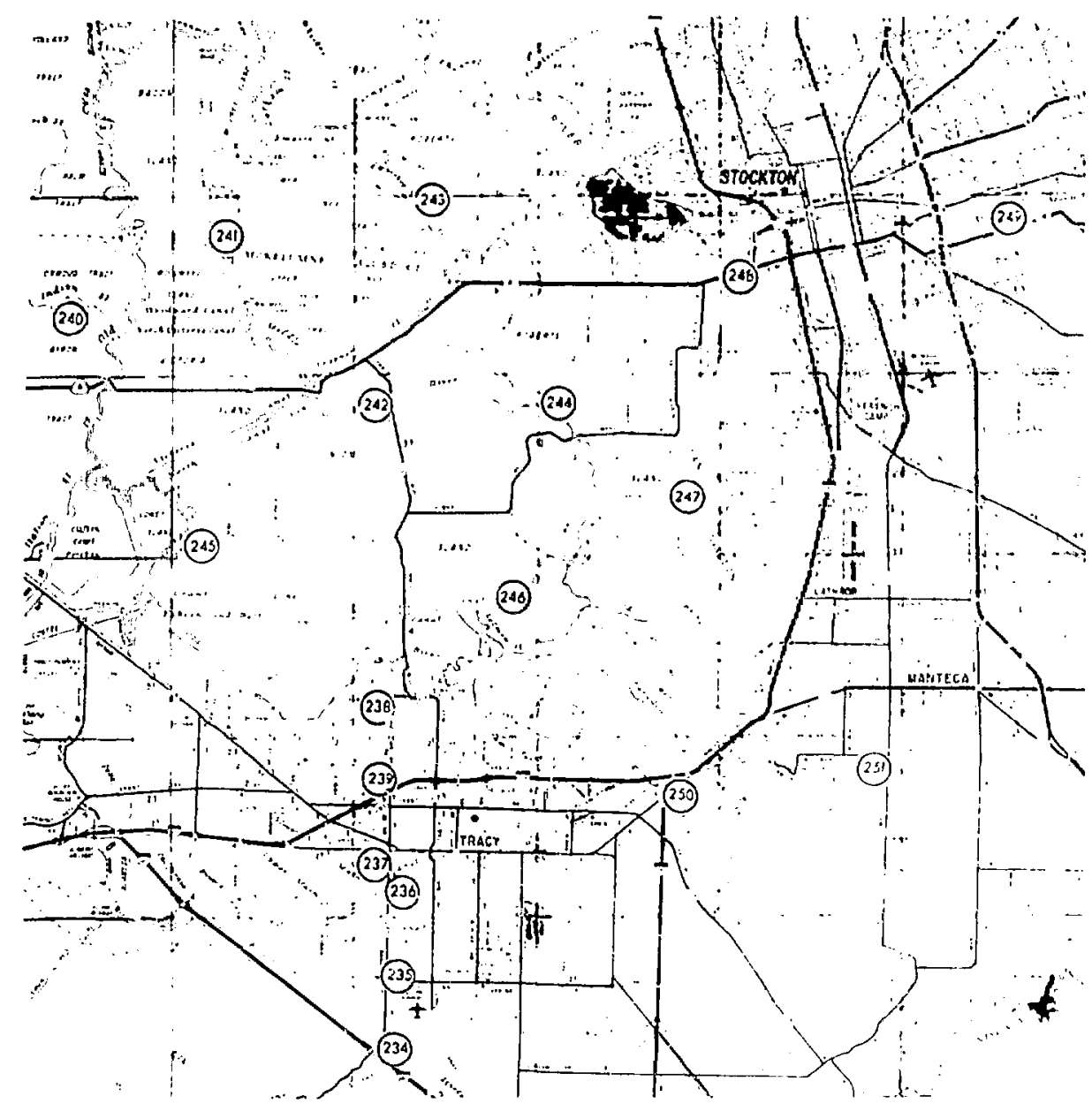

Fig. 6. Locations of soil samples collected in the San Joaquin Valley.

the San Joaquin samples correspond to those for natural uranium, Site 300 operations are not a credible source for these elevated levels.

Table 7 shows the activity levels of ${ }^{239} \mathrm{Pu},{ }^{238} \mathrm{Pu}$, and ${ }^{137} \mathrm{Cs}$ present in ditches and arroyos which drain 1.L.L surface water runoff. Sample lorations are shown in Fig. 7. The datil show that ${ }^{239}$ pad concentrations :ae in the range previously observed in soil within the Livermore Valley. 


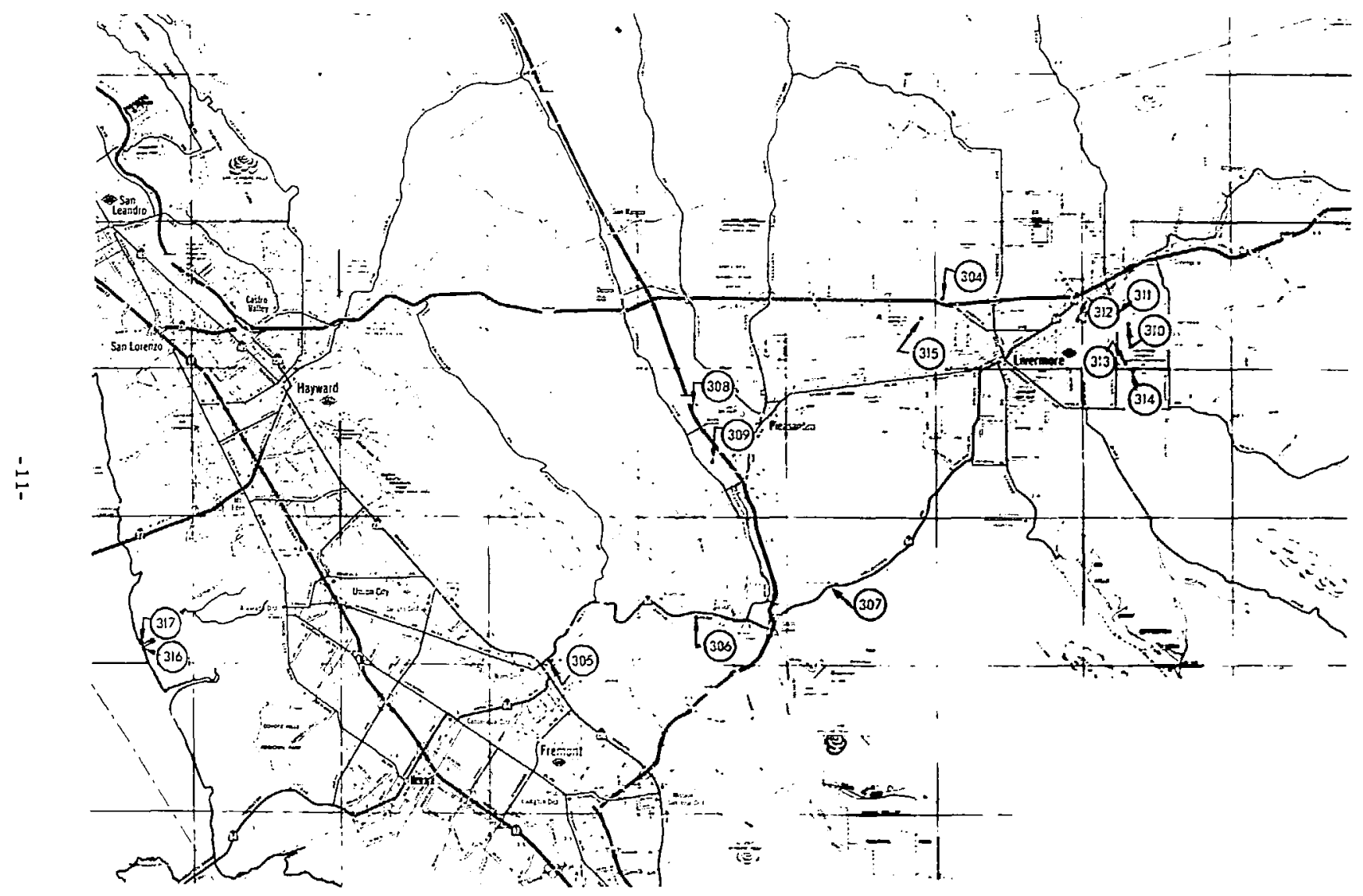

Fig. 7. Locations of sediment samples collected in ditches and arroyos into which LLL surface water drain:3. 


\section{LABORATORY SEWER EFFLUENT}

Low level radioactive wastes from the Laboratory are discharged into the City of Livermore sanitary sewer system at the LLL outfall shown in Fig. 2. This sewage is processed at the Livermore treatment plant where liquid and sludge are separated on entering the planl. Sludge is processer in no of two digesters where it is broken down by bacterial action. Digested sludge is pumped into one of two large sludge lagoons. During the summer, a portion of this sludge is removed to drying beds. The drie! sludge is used as a soil conditioner. Treated water (the plant effuent) is used for irrigating the Livermore Municipal Golf Course, lawns of the Livermore Airport, and nearby agricultural land; the excess is discharged into Mrroyo Las Positas.

Weekly samples were collected from each digester, the aeration tank, and the liquid effluent. Gross alpha and beta activities, as well as specific alpha emitting radionuclides, were measured in monthly composites of the weekly samples to determine if any significant buildup of radioactivity occurred within the plant. In addition, the activity levels of certain radionuclides in the $L L L$ effuent are compared with those in the effuent from the Livermore treatment plant. These data are shown in Tables 8 through 11 . It is seen that most of the activity is associated with the solids (sludge) in the plant. In 1973, dried sludge from one of the: lagoons showed a ${ }^{259} \mathrm{Pu}$ content of 2.6 $\times 10^{-6} \mu \mathrm{Ci} / \mathrm{g}$. This plutonium activity is related to an unintentional release to the sanitary sewer system in 1967 . The activity passed through tho sewage treat ment plant and was retained in this sludge lagoon. The supernate from the ligoon (eccondary digester supernate) is recycled through the system and, after trcatment, is released os treated effluent. Since tho plutontum content of the effluent in well below tho RCG values for drinlsing water. it is clear that the $P u$ is being retained in the sludge as expected. Detials of this relense were reported in 1967 to the Atomic Encrisy Commission, to the City of Livermore, and to the Calliornia Stute Elealth Depertment.

\section{EN VIRONMENT AL, IMPACT OF LLI LIQUID EFFLUENTS}

Except for low level radioactiva liquid wastea, which are discharged to the Livermore sanitary scvers, the Laboratory does not release radioactive liquid wastes to the environment. During 1973, the principal radionuclides relcased to the sewer were $2.0 \times 10^{-4}$ curies of $239 \mathrm{Pu}$ and 19.0 curies of ${ }^{3} \mathrm{H}$. Table 11 shows the concentrations of these radionuclides in the treated effluent from the Liv rmore Sewage Treatment $\mathrm{Plant}$. The percentages of RCG (drinking water) for ${ }^{239} \mathrm{Pu}$ and ${ }^{3} \mathrm{H}$ in this effluent were $4.3 \times 10^{-4}$ and $1.1 \times 10^{-1}$, respectively.

\section{WATER}

Water samples were collected from various locations in the Livermore Valley shown in Figs. 1 and 2. Samples were evaporated and the residues were trans ferred with dilute nitric acid to ccunting planchets. After flaming, the planchets were counted for gross alpha and beta activities in a gas proportional counter. 
No sample showed an alpha activity above the $1 \mathrm{~m}$ it of sensitivity of $1.2 \times 10^{-0} \mu \mathrm{Cl}$; $\mathrm{ml}$. The gross beta activitieg are listed in Table 12. locations 11, If through 17 and 21 through 34 are surface sources such as ponds, crecks, reservoirs, and aqueducts. Livermore rainfall is simpled at location 20. The remainder of the samples represent domestic water sources. Gross beta activitics are comparable to those observed during 1972. The highest activity was exhibited by the rainfall sumple.

These samples were also analyzed for tritium activity. Because of the low actlvities, it was neceseary to vactium diatill and elcetrolytically enrlch the samples prior to internal gas counting. The results of the analyses are shown in Table 13. Inspection of the data indicates that the simples exhibit rather uniform eritium concentrations that ara well below the recommended concentration guide value. The table also includes an eatimate of the annual dose that may be delivered to an adult consuming water containing the listed tritium concentrations. The doses, which are typically less than $0.1 \mathrm{mrem}$, are based upon a daily water consumption of 1 liter per day and the rodel of Anspaugh et al. ${ }^{6}$

\section{VEGETATION}

Vegetation samples (usually native grassey) were collected at monthiy intervals at the locations shown in Figs 1 and 2. A portion of each sample was freeze dried and the tritium activity of the recovered water was determined by liquid scintillation counting. The balance of the samples was combined to represent a monthy composite a ample. After oven drying, this composite sample was analyzed for vartoug gamma ersitung ridionuclides with the Go(Li) detector used for andaly zing the airborne partlculate samploo.

The tritium activities, ghown in Table 14 , indicate tign'ficont variation from one location to another. Is the prevaling wind is from the touthwest, one may expect the effect of the Lahoratory's operation to be minimal at Locations 4, 7, 13, 17, and 18. Tritium levels found in vegetation collected from these areas may be considered to represent normal environmental background. However, Locations 5, 28, 29, and 30. which show clevated levels, are situated in a generally downwind direction from the Laboratory. Conscquently, vegetation In these areas, as well as Locations 10 and 11 (on-site), may be exposed to the low level tritium efluent released from the Gaseoug Chemistry Bullding. The samples collected at Location 12 represent grass that has been watered by the liquid effluent from the Livermore Sewage Treatment Plant. The whole body radiation doses shown in the table were derived from the model of Anspaugh et al., assuming that the observed tritlum activIttes were typical of those in edible vetetation grown in this area. The doses arc based upon the direct daily consumption of $400 \mathrm{~g}$ of vegetation, ${ }^{7}$ which is normally $80 \%$ water. It is evident that even those samples, with elevated tritlum concentra. tlons, provide rather small whole body radiation doses.

The results of the gamma spectral analyses are shown in Table 15. Again, if one makes the assumption that the observed activities are typical of those in 


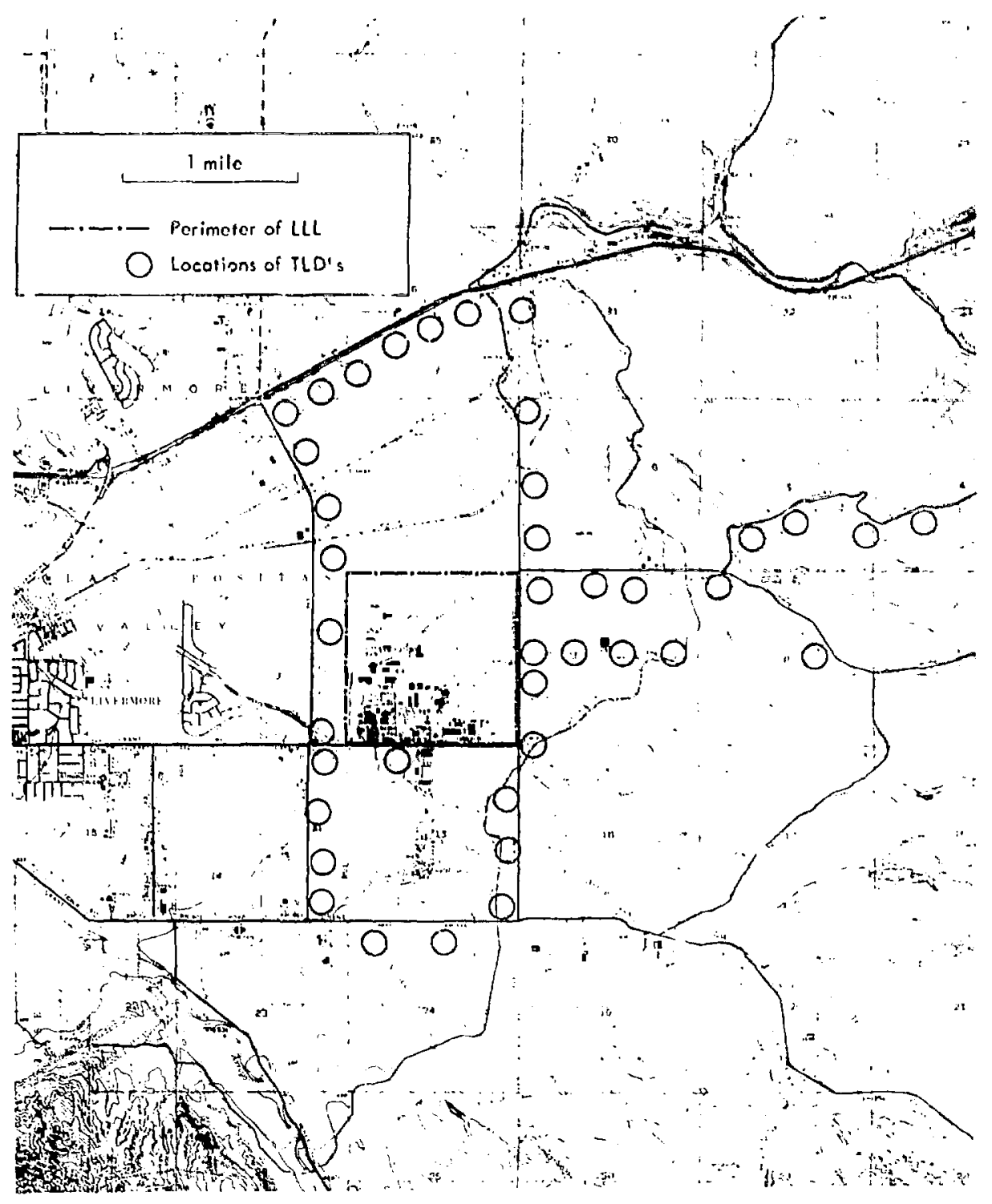

Fig. 8. Location of thermoluminescent dosimeters in the vicunity of the Liwrence Livermore Laboratory. 
edible vegetation, one may calculate the antiusl whole body or critical srgan doses to an adult resulting from the direct ingestion of these radionuclides. These calculated doses, also shown in Table 15 , are based upon an adult consumption of 400 g per day of vegetation with a molsture content of $80_{\%}^{\%}$ and the data of $\mathrm{N}_{\mathrm{g}}$ et al. ${ }^{8}$ regarding the dose recelved per unit of rallionetivity consumed under cquilibrium conditions. The naturally occurring ${ }^{40} \mathrm{~K}$ activity delivers about $25 \mathrm{mrem} / \mathrm{yr}$ to the whole body, while all the rost deliver appreciably less tlatan $1 \mathrm{mrem} / \mathrm{yr}$.

\section{ENVIRONMENTAT, RAIIATION MEASUREMENTS}

Envlronmentil radiation background measurements were made at 11 Lnboratory perimeter locations shown in Fig. 2, and at 41 off-sitc locations (shown in Fig. 8) in the vicinity of the Labor: tory. These measurements were made with $\mathrm{CaF}_{2}$ :Dy (TLJ,-200) thermoluminescent dosimeters placed at a height of approximately $1 \mathrm{~m}$ above the ground. Exposure periods were 3 mo. Based on past expericnce, the environmental terrestrial exposure rates in the Livermore Valley vary between 3 and $7 \mu R / p r$ depending on the location; cosmic radiation, calculated from the local elevation and geomagnetic latitude according to the data of Lowder and Beck, ${ }^{9}$ is approximately $4 \mu \mathrm{R} / \mathrm{hr}$. Table 16 shows quarterly and annual dose rates in mrem

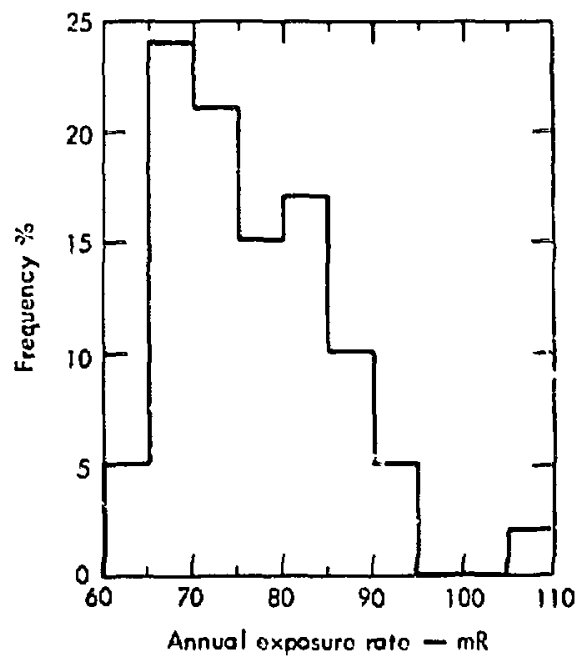

Fig. 9. Annual off-site environmental radiation exposure rates measured during 1973.

derived from the observed exposure rates at perimeter locations. Above average exposure rates were observed at Locations 5,14 , and 15. Location 5 is adjacent to a cyclotron building and Locations 14 and 15 are near a linear acceler tor facility. Figure 9 shows an annual frequency distribution of exposure rates measured at the 41 off-site locations. The median exposure rate of $74 \mathrm{mR}$ (equivalent to $71 \mathrm{mrem}$ ) shown for 1973 is comparable to the off-site median of $77 \mathrm{mR}$ observed from the 1972 data. 


\section{Environmental Levels of Radioactivity - Site 300}

\section{ATMOSPIEIR, RADIOACTIVITY}

The concentrations of various particulate radicactive substances werc measured continuously at 11 air sampling locations as shown in Figs, 10 and 11 . Ten of the samplers are located within the boundaries of site 300 and the cleventh is locited in Tracy, the primary population center of concern. The on-site samples werc collected on $8 \times 10$-in. Whatman-41 silters at a $\Omega n w$ rate of about 25 cím. Saniples in Tracy were collected on $4 \times 9-$ in. HV-70 (cellulose-asbestos) filters at a flow rate of about $4 \mathrm{cfm}$. Filters were chenged twice a week during the summer months to avoid excessive mass loading; during the ballance of the year they were changed on a weekly basis. The filters were analyzed by the methods pre iously described for air filters used in the Livermore Valley.

No gross alpha activity above the $1 \times 10^{-15} \mu \mathrm{Ci} / \mathrm{ml} \mathrm{dr}+$ ction limit was observed on these filters. The gross beta activities, averaged over 6-mo periods, are !isted in Table 17 for each sampling location. Figure 12 shows the weekly average activities deposited on air filters from all the sampling locations. These average activities are slightly higher than those measured in the Livermore Valley, but are lower than the values observed during past years. As previously noted, these reductions are due to a decrease in the level of global fallout radioactivity in th. 3 at mosphere.

Gamma spectral measurements made on monthly composite samples of the filters collected on-site reveal measurable quantities of virrous gamma-emitting radionuclides as may be seen in Table 18 . These activity levels are essentally identical with those measured in the l.ivermore valley ad may readily bc iccounted for as being due to global fallout. The results of isotopic plutionium and uranium analyses, performed by the techniques described previously, are provided in Table 19. The relative abundance of ${ }^{238} \mathrm{Pu}$ to ${ }^{239} \mathrm{Pu}$ is approximately that expected in global fallout. The uranium, on the other hand, is appreciably depleted in $235 \mathrm{U}$ relative to that of natural uranium becuuse the amount used in high explosives experiments has been speclally processed to lower the ${ }^{235} \mathrm{U}$ content. Although the concentrations are at times higher than those measured in air in the Livermore Valley, they are nevertheless far lower than the AEC concentration guide levels.

\section{ECOLOGY IMPACT STUDY}

An ecology study was initiated during the summer of 1973 to determine if Site 300 operations have a measurable impact on plants and animals native to this area. The work is part of the ongoing program of environmental surveillance and potential impact analysis of the site. Soils, plants, and animals are being collected from various locations on the site and analyzed for uranium and other elements of possible impact. Emphasis is being placed upon areas around the high explosive firing bunkers. For purposes of comparison, similar plants and animals are being collected from surrounding offsite locations. This work is continuing 


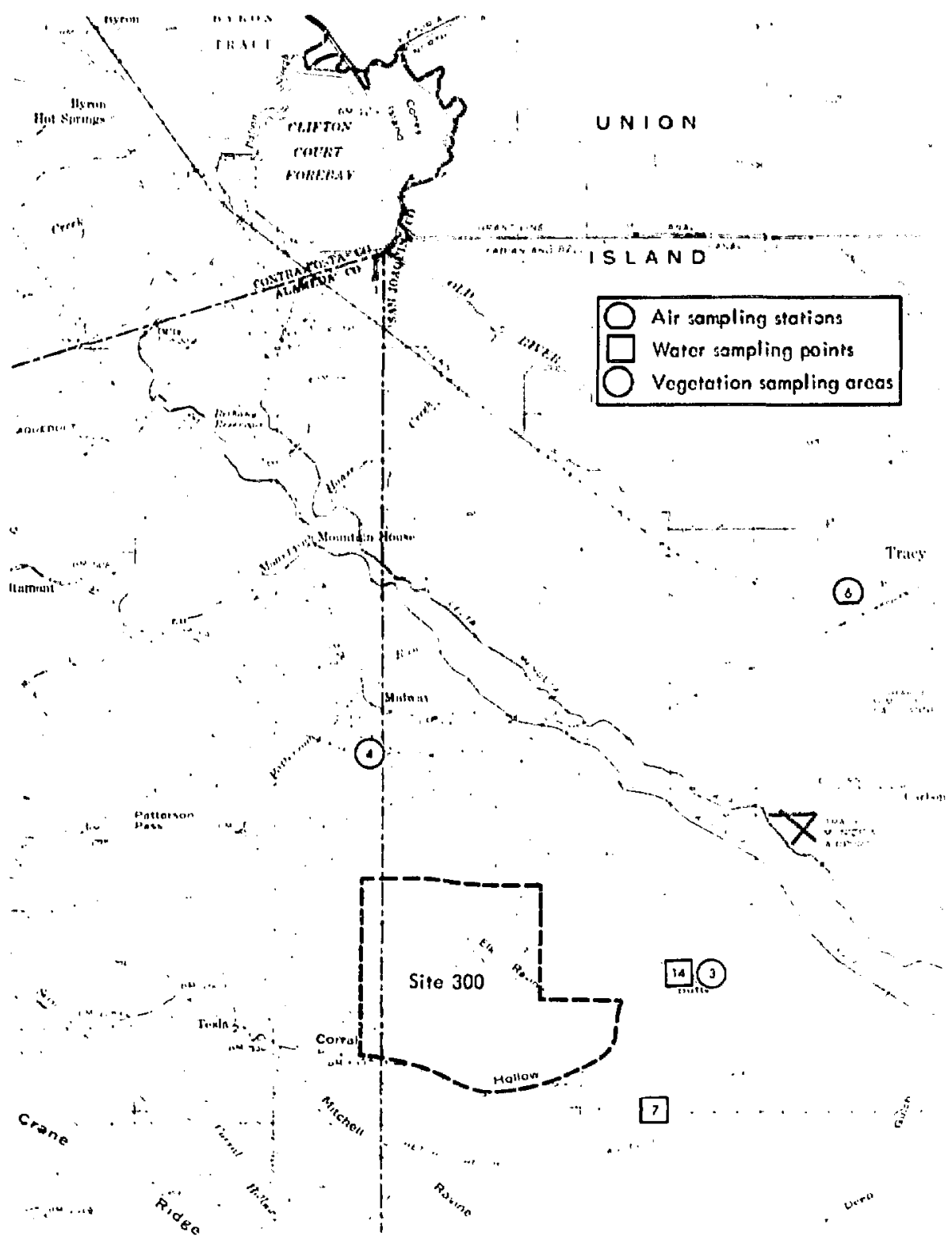

Fig. 10. Air, water, and vegetation sampling locations in the Site 300 area. 


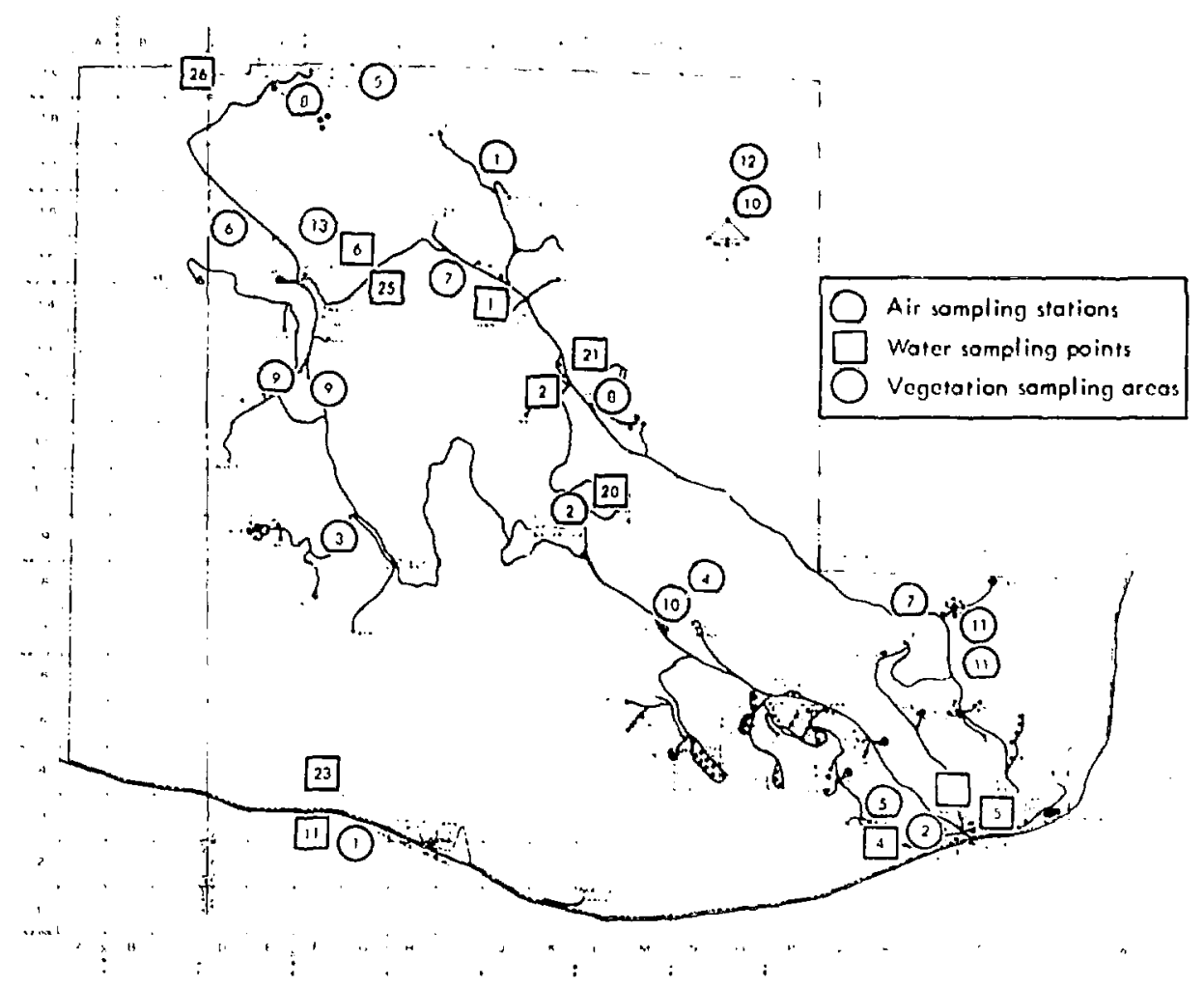

Fig. 11. Air, water, and vegetation sampling locations inside Site 300 boundary.

and will be reported in our 1974 annual report.

SOIL

High explosive tests at Site 300 often involve the use of "depleted" uranium (uranium in which the uranium is processed to reduce the $235 \mathrm{U}$ content). In 1972, a special study was conducted to determine the extent to which the natural ${ }^{235} \mathrm{U} /{ }^{238} \mathrm{U}$ ratio in Site 300 soil was perturbed by these operations. Based on mass spectrometric isotopic uranium analysis of soils ccllected throughout the test site, it was found that depletion of ${ }^{235} U$ was essentially restricted to areas adjacent to the firing bunkers. ${ }^{2}$ Similar surveillance was maintained in 1973, but the number of samples was reduced to that level considered adequate to document any significant change, which might have occurred since the 1972 study. These samples were collected to a depth of one centimeter using the same sampling procedure and soil preparation technique described for Livermore Valley samples. 


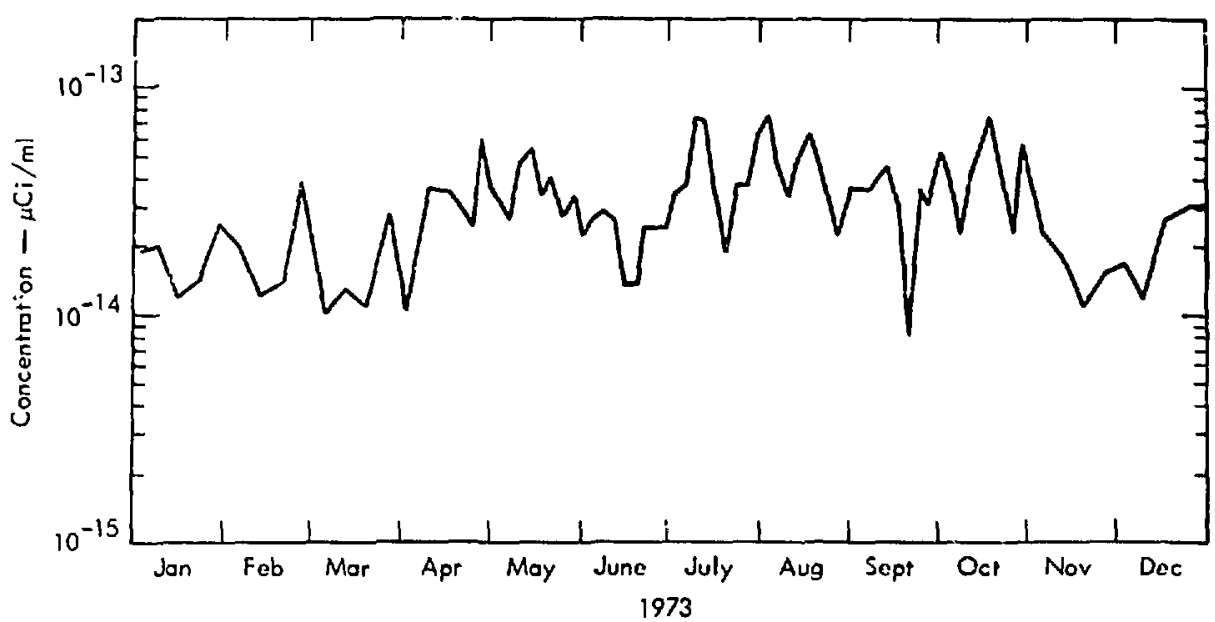

Fig. 12. Weekly average airborne gross beta activity on air filters from Site 300 sampling stations.

Sample locations are shown in Fig. 13 and the results of the isotopic uranium analyses are presented in Table 20. These data are comparable to those observed in 1972.

\section{WATER.}

Samples were collected from on-site wells supplying Site 300 and from various on-site and off-site springs, ponds, and creeks. The locations of these sites are shown in Figs. 10 and 11 . Locations 1 througl 7 represent deep-well sources, Locations 11 and 14 are off-site creek sources, and rain water is collerted at Location 20. The remaining locations are on-site ponds or springs. The samples were subjected to gross alpha and beta analyses. No samples showed a gross alpha activity above the limit of detection of $1.2 \times 10^{-9} \mu \mathrm{Ci} / \mathrm{ml}$. The gross beta activities, averaged over G-mo periods, are given in Table 21 . These activities show little variation with time and location and are similar to those exhibited by the water samples collected within the Livermore Valley. The samples were also subjected to tritium analyses by electrolytic enrichment and subsequent internal gas counting. The results of the analyses are shown in Table 22. With the exception of the deep well waters, which show very little tritium, these samples contain tritium activities that are comparable with those observed in water collected within the Livermore Valley.

Table 22 also includes the calculated whole body doses to an adult consuming the water containing the listed tritium concentrations. These joses were derived in the same manner used to compute the values listed in Table 13. 


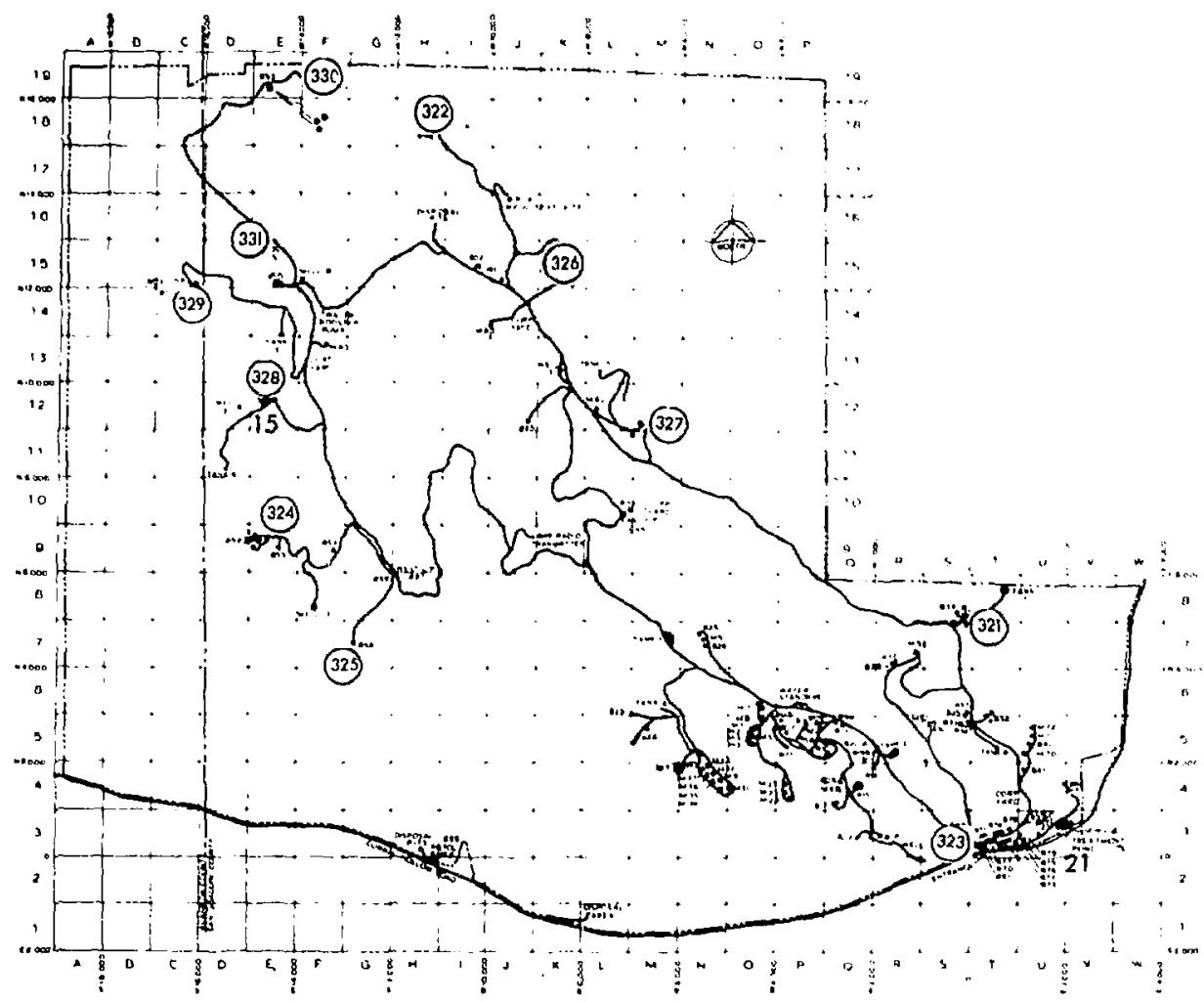

Fig. 13. Site 300 soil sampling locations during 1973.

\section{VEGETATION}

Vegetation samples were collected on a monthly basis at the 13 locations shown in Figs. 10 and 11. Dried monthly composite samples were subjected to gamma spectral analyses yielding the activities shown in Table 23. Also shown in the table are the calculated whole body or critical organ doses delivered to an adult by direct ingestion of $400 \mathrm{~g}$ per day of edible vegetation containing $80 \%$ water and the listed a... age activities. The data reveals that natural ${ }^{40} \mathrm{~K}$ and ${ }^{144} \mathrm{Ce}$ from global fallout are the major contributors via this pathway.

Theso samples were also subjected to tritium analyses by freeze drying and scintillation counting of the resulting water. The results are shown in Table 24. The activities show little fluctuation compared with the results obtained from similar samples collected within the Livermore Valley. The table also includes the resulting whole body doses delivered to an adult by the tritium activities based upon 
the models referenced previously. As one would expect, these doses are insignificant.

MILK

The only dairy in the general vicinity of Laboratory operations is located about $6 \mathrm{mi}$ southwest of Tracy. Periodic milk samples were collected from the dairy throughout the year. Before analysis, the samples were concentrated by means of freeze drying and the concentrates were gamma counted in a $\mathrm{Ge}(\mathrm{Li})$ counting system. In addition, each sample was ana- lyzed for tritium activity by counting $1 \mathrm{ml}$ of the water recovered from freeze drying in a liquid scintlllation counting system. Activities of ${ }^{137} \mathrm{Cs},{ }^{40} \mathrm{~K}$, and ${ }^{3} \mathrm{H}$ are shown in Table 25. No other radionuclides were detected. Also shown are the $\mathrm{calcu}$ lated annual adult whole body or critical organ radiation dose delivered to man via the milk pathray. These calculations are based on a dally intake of $260 \mathrm{~g} / \mathrm{day}$ and the models previously referenced. As expected, the only aignificant dose to an individual is that from naturally occurring ${ }^{40} \mathrm{~K}$. 
Table 1. Airborne particulate beta radioactivity within the Livermore Valley during $1973(\mu \mathrm{Ci} / \mathrm{ml})$.

\begin{tabular}{|c|c|c|c|c|c|c|c|c|c|c|}
\hline \multirow{2}{*}{$\begin{array}{l}\text { Sampling } \\
\text { location }\end{array}$} & \multirow{2}{*}{$\begin{array}{r}\text { No. of } \\
\text { samples }\end{array}$} & \multicolumn{3}{|c|}{$J$ anuary-June } & \multirow{2}{*}{$\begin{array}{c}\text { No. of } \\
\text { samples }\end{array}$} & \multicolumn{3}{|c|}{ July-necember } & \multirow{2}{*}{$\begin{array}{l}\text { Annual } \\
\text { average }\end{array}$} & \multirow{2}{*}{ RCG } \\
\hline & & Maximum & Minimum & Average & & Maximum & Minimum & Aver age & & \\
\hline 1 & 22 & $3.9 \times 10^{-14} \pm 6 \%$ & $1.6 \times 10^{-15} \div 67 \%$ & $1.6 \times 10^{-14}$ & 24 & $3.8 \times 10^{-14} \pm 5 \%$ & $1.0 \times 10^{-15} \pm 100 \%$ & $<2.0 \times 10^{-14}$ & $<1.8 \times 10^{-14}$ & 2 \\
\hline 2 & 23 & $4.7 \times 10^{-14} \pm 7 \%$ & $6.4 \times 10^{-15} \pm 24 \%$ & $2.0 \times 10^{-14}$ & 21 & $3.2 \times 10^{-14} \pm 79$ & $5.2 \times 10^{-15} \pm 23 \%$ & $2.2 \times 10^{-14}$ & $2.6 \times 10^{-14}$ & 2 \\
\hline 3 & 25 & $3.2 \times 10^{-14} \pm 13 \%$ & $3.9 \times 10^{-15} \pm 100 \%$ & $41.6 \times 10^{-14}$ & 22 & $5.6 \times 10^{-14}+9^{\%}$ & $4.1 \times 10^{-15} \pm 100 \%$ & $<2.0 \times 10^{-14}$ & $4.8 \times 10^{-14}$ & 2 \\
\hline 4 & 25 & $7.5 \times 10^{-14} \pm 7 \%$ & $9.9 \times 10^{-15} \pm 34_{\%}^{\sigma}$ & $2.9 \times 10^{-14}$ & 21 & $8.5 \times 10^{-14} \pm 13 \%$ & $1.4 \times 10^{-14} \pm 24 \%$ & $3.7 \times 10^{-18}$ & $3.3 \times 10^{-14}$ & 3 \\
\hline 5 & 26 & $2.9 \times 10^{-14} \pm 14 \%$ & $4.5 \times 10^{-15} \pm 22^{\%}$ & $1.5 \times 10^{-14}$ & 24 & $4.4 \times 10^{-14} \pm 9 \%$ & $4.2 \times 10^{-15} \pm 100^{\sigma}$ & $<1.9 \times 10^{-14}$ & $<1.7 \times 10^{-14}$ & 2 \\
\hline 6 & 25 & $4.8 \times 10^{-14} \pm 10 \%$ & $4.5 \times 10^{-15} \pm 100 \%$ & $<1.4 \times 10^{-14}$ & 24 & $5.2 \times 10^{-14} \pm 11 \%$ & $5.9 \times 10^{-15} \pm 50 \%$ & $2.1 \times 10^{-14}$ & $<2.8 \times 10^{-14}$ & 2 \\
\hline 7 & 25 & $\therefore .6 \times 10^{-14} \pm 12 \%$ & $3.9 \times 10^{-15}+100 \%$ & $=1.7 \times 10^{-14}$ & 21 & $4.6 \times 10^{-14} \pm 19 \%$ & $4.2 \times 10^{-15} \pm 100 \%$ & $\therefore 2.1 \times 10^{-14}$ & $<1.9 \times 10^{-19}$ & 2 \\
\hline 8 & 23 & $4.8 \times 10^{-14} \pm 10 \%$ & $4.5 \times 10^{-15} \pm 100 \%$ & $<1.3 \times 10^{-14}$ & 26 & $3.9 \times 10^{-14} \pm 22 \%$ & $4.2 \times 10^{-15} \pm 100 \%$ & $<1.3 \times 10^{-14}$ & $<1.5 \times 10^{-14}$ & 2 \\
\hline 9 & 26 & $6.2 \times 10^{-14} \pm 8 \%$ & $1.3 \times 10^{-15} \pm 25_{a}^{0}$ & $3.0 \times 10^{-14}$ & 2.3 & $9.0 \times 10^{-14} \neq 12 \%$ & $1.8 \times 10^{-14} \pm 19 \%$ & $4.2 \times 10^{-14}$ & $3.6 \times 10^{-14}$ & 4 \\
\hline 10 & 24 & $1.3 \times 10^{-14} \pm 24 \%$ & $4.5 \times 10^{-15}+100^{\%}$ & $<1.3 \times 10^{-14}$ & 25 & $5.5 \times 10^{-14} \pm 17 \%$ & $4.3 \times 10^{-15} \pm 67 \%$ & $1.8 \times 10^{-14}$ & $<1.6 \times 10^{-14}$ & 2 \\
\hline 11 & 21 & $2.8 \times 10^{-14} \pm 15 \%$ & $3.6 \times 10^{-15} \pm 100^{\sigma}$ & $1.3 \times 10^{-14}$ & 26 & $4.4 \times 10^{-14} \neq 20 \%$ & $4.2 \times 10^{-15} \pm 100 \%$ & $-2.0 \times 10^{-14}$ & $<1.7 \times 10^{-14}$ & 2 \\
\hline 12 & 25 & $4.2 \times 10^{-14} \pm 7 \%$ & $9.5 \times 10^{-15}+15 \%$ & $2.2 \times 10^{-14}$ & 25 & $9.4 \times 10^{-14} \pm 5 \%$ & $7.8 \times 10^{-15}+15 \%$ & $2.7 \times 10^{-14}$ & $2.5 \times 10^{-14}$ & 3 \\
\hline 13 & 23 & $5.0 \times 10^{-14} \pm 6 \sigma_{13}$ & $3.8 \times 10^{-15} t 44 \%$ & $2.1 \times 10^{-14}$ & 20 & $3.8 \times 10^{-14} \pm 5 \%$ & $4.0 \times 10^{-15} \pm 21 \%$ & $1.9 \times 10^{-14}$ & $2.0 \times 10^{-14}$ & 2 \\
\hline 14 & 26 & $3.3 \times 10^{-14} \pm 6 \sigma$ & $7.1 \times 10^{-15} \pm 22 \sigma_{v}$ & $1.8 \times 10^{-14}$ & 23 & $3.6 \times 10^{-14}=6 \%$ & $5.6 \times 10^{-15} \neq 18 \%$ & $2.0 \times 10^{-14}$ & $1.9 \times 10^{-14}$ & 2 \\
\hline 15 & 25 & $7.7 \times 10^{-14} \pm 9 \%$ & $2.0 \times 10^{-15} \pm 54^{0 i}$ & $2.1 \times 10^{-14}$ & 26 & $+.1 \times 10^{-14}+5 \%$ & $4.7 \times 10^{-15} \pm 20 \%$ & $2.2 \times 10^{-14}$ & $2.2 \times 10^{-14}$ & 2 \\
\hline 16 & 24 & $3.6 \times 10^{-14} \pm 6 \%$ & $5.9 \times 10^{-15} \pm 22 \%$ & $2.0 \times 10^{-14}$ & 20 & $4.3 \times 10^{-24} \pm 6 \%$ & $1.3 \times 10^{-14} \pm 12 \%$ & $2.5 \times 10^{-14}$ & $2.3 \cdot 10^{-1.4}$ & 2 \\
\hline 17 & 10 & $5.0 \times 10^{-14} \pm 6 \%$ & $7.5 \times 10^{-15} \pm 16^{0}$ & $2.2 \times 10^{-14}$ & 20 & $5.1 \times 10^{-14} \pm 6 \%$ & $9.5 \times 10^{-15} \pm 14 \%$ & $2.5 \times 10^{-14}$ & $2.4 \times 10^{-1 . t}$ & 2 \\
\hline & Average & & & $1.9 \times 10^{-14}$ & & & & $<2.3 \times 10^{-14}$ & $<2.1 \times 10^{-14}$ & 2 \\
\hline
\end{tabular}

${ }^{a}$ See Figs, 1 and 2 for sampling locations.

${ }^{b}$ The RCG (Radioactivity Concentration Guide) for airborne beta is $\mathrm{I} \times 10^{-12} \mu \mathrm{Ci} / \mathrm{m} 1$. 
Table 2. Results of gamma-ray spectral measurements of Livermore Laboratory perimeter air filters during 1973 ( $\mu$ Ci $/ \mathrm{ml}$ ).

\begin{tabular}{|c|c|c|c|c|c|c|c|c|c|}
\hline Mronth & ${ }^{1+4} \mathrm{Ce}$ & ${ }^{141} \mathrm{Cn}^{a}$ & $125 \mathrm{Sh}$ & ${ }^{7} \mathrm{Br}$ & $10 . \mathrm{Hl}_{\mathrm{Hu}}$ & 105114 & ${ }^{137} \mathrm{CS}$ & $\because \%$ & ${ }^{10} \mathrm{~K}$ \\
\hline Ian. & $1.7 \times 10^{-15}: 31 \%$ & - & $4.0 \times 10^{-16} \cdot 76 j^{\prime r}$ & $8.0 \times 10^{-14}+44^{2}$ & - & - & $5.1 \times,,^{-15}, 35$ & $7.3 \times 10^{-16} \cdot 98^{\circ}$ & $-b$ \\
\hline Frh. & $3.0 \times 10^{-15}: 14^{\tau}$ & - & $2.3 \times 10^{-16} \cdot 36^{2}$ & $\therefore 1 \times 10^{-13}: 4^{n}$ & - & $2.7 \times 10^{-16} \cdot 56^{n}$ & $1.0 \times 10^{-15} \cdot 17^{x}$ & $5.9 \times 10^{-16} \cdot 70^{x}$ & $-^{b}$ \\
\hline Mar. & $3.3 \times 10^{-15} \div 13^{n}:$ & $3.7 \times 10^{-17}+100^{7}$ & $4.5 \times 10^{-16} \cdot 57 r$ & $1.1 \times 10^{-13} \div 4$ & $1.0 \cdot 10^{-16} \quad 100^{2}$ & $1.7 \times 10^{-15}: 86 \%$ & $1.1 \times 10^{-15} \cdot 10^{-}$ & $3.3 \times 10^{-16}-6.58$ & $-{ }^{t}$ \\
\hline$\Lambda \mathrm{pr}$. & $3.6 \times 10^{-15}: 9 m_{n}$ & - & $4.5 \times 10^{-16}, 12^{4}$ & $1.3 \times 10^{-13} \cdot 2$ & $2.4 \times 10^{17}=82$ & $2.18 \times 10^{-17} \cdot 160^{\circ}$ & $1.5 \times 10^{-15}+4$ & $7.5 \times 10^{-17}: 26=$ & $7.0 \times 10^{-11,} \cdot 2 \mathrm{n}^{\mathrm{F}}$ \\
\hline May & $2.4 \times 10^{-1.5}=10 \%$ & - & $3.7 \times 10^{-16} \cdot 12 \pi$ & $1.0 \times 10^{-13}: 2$ & - & $1.8 \times 10^{-15}: 13^{2}$ & $1.3 \times 10^{-15}+5^{2}$ & $7.2 \times 10^{-17} \cdot 72^{2 \pi}$ & $5.7 \times 10^{-16} \times 30^{7}$ \\
\hline June & $1.2 \times 10^{-15}+2 n+$ & - & $2.1 \times 10^{-16} \cdot 1 \mathrm{kP}^{2}$ & $7.4 \times 10^{-14} \div 2^{2 r}$ & - & $1.1 \times 10^{-15}: 16^{2}$ & $7.0 \times 10^{-16}, 6 \%$ & $2.3 \times 10^{-17} \cdot 100^{2}$ & $8.7 \times 10^{-16}+24^{20}$ \\
\hline tuly & $1.4 \times 10^{-15}+14 \%$ & $1.2 \times 10^{-15}: 12^{\mathrm{m}}$ & $1.8 \times 10^{-16}+19^{\alpha}$ & $7.3 \times 10^{-14}=10^{17}$ & $2.9 \times 10^{-1.7} \cdot 1 \mathrm{H}^{\mathrm{T}}$ & $1,4 \times 10^{-15}: 12^{*}$ & $7.2 \times 10^{-15} \cdot 10^{\circ}$ & $4,6 \times 10^{-16} \pm 1 ! r$ & $1.6 \times 10^{13} \cdot 11^{3}$ \\
\hline Aug. & $9.5 \times 10^{-16}+19 \%$ & $7.1 \times 10^{-16}=14 \pi$ & $1.4 \times 10^{-16 i}=30^{\pi}$ & 1.6.6.10 $10^{-14}+10^{n}$ & $1.2 \times 10^{-17} \cdot 11^{7}$ & $9.7 \times 10^{-16}: 24^{-1}$ & $5.3 \times 10^{-16} \cdot 10$ & $7.4 \times 10^{-16} \cdot 6^{r}$ & $1.4 \times 10^{-15}: 22^{2}$ \\
\hline Sept. & $7.2 \times 10^{-16}: 25 \%$ & $4.8 \times 10^{-16}+13 \%$ & $1 .+\times 10^{-16} \div 42^{x}$ & $5.5 \times 10^{-14}=5$ & $1.0 \times 10^{-1 \%}, 6$ & $5.4 \times 10^{-16} \cdot 20^{2}$ & $3.3 \times 10^{-16}: 14^{m}$ & $8.2 \times 10^{-16} \times$ & $1.1 \times 10^{-15}: 21^{r}$ \\
\hline Oct. & $1.4 \times 10^{-15}+17^{n}$ & $1.3 \times 10^{-15}: 1.4 \%$ & $\overline{17}$ & $1.2 \times 10^{-13} \div 7^{5}$ & $2.3 \times 10^{-15}: 20^{r}$ & $1.1 \times 10^{-1.5}: 2.3 \sigma_{0}$ & $3.4 \times 10^{-16}: 6^{\%}$ & $2.6 \times 10^{-15}, 2^{2}$ & $1.0 \times 10^{-15} \pm 31 \%$ \\
\hline Nov. & $1.2 \times 10^{-15}: 16^{\circ} \mathrm{c}$ & $7.9 \times 10^{-16}+11 \%$ & $9.0 \times 10^{-17}, 52^{r}$ & $6.3 \times 10^{-14}+4^{n}$ & $1.4 \times 10^{-1.5}+8$ & $7.5 \times 10^{-15_{i}}, 32 \tau$ & $1.7 \times 10^{-16} \cdot 2.1^{n}$ & $2.0 \times 10^{-15}+50^{2}$ & $7.3 \times 10^{-16} \cdot 25$ \\
\hline $\begin{array}{l}\text { Bec. } \\
\text { Annual av }\end{array}$ & $\begin{array}{l}2.7 \times 10^{-15}+9^{r} \\
2.0 \times 10^{-15}\end{array}$ & $1.1 \times 10^{-15}=87:$ & $1.2 \times 10^{-16}+4 ! n$ & $8.6 \times 10^{-14} \div 4^{r}$ & $2.0 \times 10^{-15}:=n$ & $1.8 \times 10^{-15} \cdot 16 \%$ & $3.2 \times 10^{-16}+127$ & $3.5 \times 10^{-15} \cdot 15^{*}$ & $7.2 \times 10^{-16} \cdot 30^{\circ}$ \\
\hline Annual av & $2.0 \times 10^{-10}$ & & $2.6 \times 10^{-718}$ & $8.5 \times 10^{-14}$ & $1.3 \times 10^{-17}$ & $1.2 \times 10^{-15}$ & $7.1 \times 10^{-16}$ & $1.1 \times 10^{-15}$ & $9.6 \times 10^{-16}$ \\
\hline $\begin{array}{l}\mathrm{RCO} \\
0 \mathrm{RCG}\end{array}$ & $\begin{array}{l}2.0 \times 10^{-3} \\
1.0 \times 10^{-3}\end{array}$ & & $0.0 \times 10^{-10}$ & $4 . \Gamma \times 10^{-5}$ & $3.0 \times 10^{-41}$ & $2.0 \times 10^{-10}$ & $5.0 \times 10^{-10}$ & $1.0 \times 10^{-11}$ & $+515^{-7}$ \\
\hline RCG & & $1.6 \times 10^{-5}$ & $2.9 \times 10^{-5.3}$ & $2.1 \times 10^{-4}$ & $4.3 \times 10^{-5}$ & $5.11 \times 10^{-4}$ & $1.4 \times 10^{-4}$ & $1.1 \times 10^{-4}$ & $2.5 \times 10^{-5}$ \\
\hline
\end{tabular}

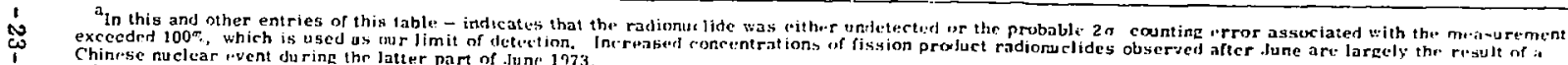

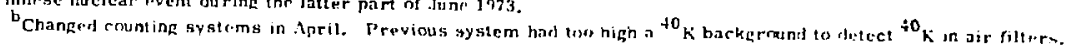



Table 3. Plutonium, Americium, Strontium, and Uranium in air at Livermore Laboratory perimeter locations during 1973
$(\mu \mathrm{Ci} / \mathrm{ml})$.

\begin{tabular}{|c|c|c|c|c|c|c|c|c|c|c|}
\hline \multirow[b]{2}{*}{ Month } & \multirow[b]{2}{*}{ Loration } & \multirow[b]{2}{*}{${ }^{239} \mathrm{Pu}$} & \multirow[b]{2}{*}{${ }^{238} \mathrm{Pu}$} & \multirow[b]{2}{*}{${ }^{238} \mathrm{Pu} /{ }^{239} \mathrm{Pu}$} & \multirow[b]{2}{*}{$241 \mathrm{Am}$} & \multirow[b]{2}{*}{${ }^{30} \mathrm{Sr}$} & \multirow[b]{2}{*}{${ }^{239} \mathrm{Pu} /{ }^{10} \mathrm{Sr}$} & \multicolumn{2}{|c|}{ Mass $\left(\mathrm{mg} / \mathrm{M}^{3}\right)$} & \multirow[b]{2}{*}{$235_{\mathrm{C}}{ }^{238_{\mathrm{r}}}$} \\
\hline & & & & & & & & 2.35 & 238 & \\
\hline \multirow[t]{6}{*}{ Jan. } & 1 & $7.8 \times 10^{-18}+15 \%$ & - & & $6.4 \times 10^{-19} \pm 34+$ & $3.7 \times 10^{-16}:: 2^{r}$ & $2.1 \times 10^{-2}$ & $2.2 \times 10^{-10} \cdot+^{\tau}$ & $3.5 \times 10^{-8}: 5^{T}$ & $0.3 \times 10^{-3}$ \\
\hline & 2 & $9.7 \times 10^{-18} \div 13 \%$ & - & & $4.5 \times 10^{-19}: 40^{m_{n}}$ & $4.3 \times 10^{-16}+12 x$ & $2.3 \times 10^{-2}$ & $2.8 \times 10^{-10}: 3^{r}$ & $4.1 \times 10^{-8}: 4 \%$ & $6.8 \times 10^{-3}$ \\
\hline & 12 & $6.4 \times 10^{-18}+13 \%$ & - & & $5.7 \times 10^{-19}: 46^{m}$ & $3.9 \times 10^{16}, 9 \%$ & $1.6 \times 10^{-2}$ & $2.8 \times 10^{-10}, 3^{\tau}$ & $4.4 \times 10^{-8}+4 \%$ & $6.4 \times 10^{-3}$ \\
\hline & 13 & $2.8 \times 10^{-18}+18 \%$ & - & & $3.4 \times 10^{-19}+43^{m}$ & $1.2 \times 10^{-16}+16 \%$ & $2.3 \times 10^{-2}$ & $2.0 \times 10^{-10}: 4 \pi$ & $2.8 \times 10^{-8}: 5$ & $7.1 \times 10^{-3}$ \\
\hline & $14^{\circ}$ & $2.6 \times 10^{-17} \pm 11 \%$ & $9.1 \times 10^{-1 \%}+17 \%$ & $3.5 \times 10^{-1}$ & $2.1 \times 10^{-18}+56_{r}^{m}$ & $8.2 \times 10^{-16} \cdot 16^{\sigma}$ & $3.2 \times 10^{-2}$ & $4.4 \times 10^{-10}=4^{n}: \ldots$ & $7.6 \times 10^{-8}+5^{m}$ & $6.3 \times 10^{-3}$ \\
\hline & 15 & $8.4 \times 10^{-18}: 16 \%$ & $7.2 \times 10^{-19}+3 ?$ & $8.6 \times 10^{-2}$ & $3.6 \times 10^{-19}: 58 \%$ & $3.7 \times 10^{-16}+16^{20}$ & $2.3 \times 10^{-2}$ & $2.6 \times 10^{-10}, 4 \pi$ & $4.1 \times 10^{-8}+50$ & $6.3 \times 10^{-3}$ \\
\hline \multirow[t]{6}{*}{ Feb. } & 1 & $4.1 \times 10^{-17}: 8 \%$ & $3.0 \times 10^{-18} \pm 22^{\%}$ & $7.3 \times 10^{-2}$ & $2.4 \times 10^{-18} \pm 32^{m}$ & $6.2 \times 10^{-16}+9 \%$ & $6.6 \times 10^{-2}$ & $3.7 \times 10^{-10}+4^{\sigma}$ & $5 .+\times 10^{-8}: 5^{\prime \prime}$ & $6.9 \times 10^{-3}$ \\
\hline & 2 & $1.4 \times 10^{-17}+9 \%$ & $1.1 \times 10^{-18} \div 24 \sigma$ & $7.6 \times 10^{-2}$ & $9.4 \times 10^{-19}+399^{2}$ & $6.6 \times 10^{-16}, 7^{\sigma}$ & $2.1 \times 10^{-2}$ & $4.2 \times 10^{-10}: 33^{n}$ & $6.1 \times 10^{-8}: 3^{x}$ & $5.9 \times 10^{-3}$ \\
\hline & 12 & $1.7 \times 10^{-17}, 8^{\sigma}$ & $2.3 \times 10^{-18}: 18$ & $1.4 \times 10^{-1}$ & - & $5.9 \times 10^{-16} \pm 5^{\sigma}$ & $2.9 \times 10^{-2}$ & $3.8 \times 10^{-10}+3 \%$ & $5.7 \times 10^{-8}+35$ & $6.7 \times 10^{-3}$ \\
\hline & 13 & $2.1 \times 10^{-27}, 8 \%$ & $1.7 \times 10^{-18}: 22^{n}$ & $8.1 \times 10^{-2}$ & - & $6.0 \times 10^{-16}: 6^{0}$ & $3.5 \times 10^{-2}$ & $3.4 \times 10^{-10}: 35$ & $4.81 \times 10^{-k}: 4^{\sigma}$ & $7.1 \times 10^{-3}$ \\
\hline & 14 & $1.1 \times 10^{-16}+5 \%$ & $4.2 \times 10^{-1} \cdot 13 \%$ & $3.8 \times 10^{-2}$ & $1.6 \times 10^{-17} \neq 10^{\sigma}$ & $4.5 \times 10^{-16} \pm 7^{\alpha}$ & $2.4 \times 10^{-1}$ & $1.7 \times 10^{-10}: 35$ & $2.7 \times 10^{-4}: 4^{r}$ & $6.6 \times 10^{-3}$ \\
\hline & 15 & $1.8 \times 10^{-17}+4 \%$ & $1.2 \times 10^{-18} \pm 26^{\sigma}$ & $6.3 \times 10^{-2}$ & $1.9 \times 10^{-18} \div 24 \pi$ & $5.4 \times 10^{-16}: 6^{5}$ & $3.5 \times: 0^{-2}$ & $2.9 \times 10^{-10} \div 3^{n}$ & $1.1 \times 10^{-8}+4^{2}$ & $7.1 \times 10^{-3}$ \\
\hline \multirow[t]{6}{*}{ Mar. } & 1 & $1.2 \times 10^{-17}+12^{\sigma}$ & $1.4 \times 10^{-18}+26^{\infty}$ & $1.2 \times 10^{-1}$ & - & $6.0 \times 10^{-16} \div 65$ & $2.0 \times 10^{-2}$ & $2.6 \times 10^{-10}: 14^{7}$ & $1.4 \times 10^{-8}=190$ & $6.4 \times 10^{-3}$ \\
\hline & 2 & $1.3 \times 10^{-17}+11 \%$ & $1.7 \times 10^{-18} \pm 24 \%$ & $1.3 \times 10^{-1}$ & - & $7.2 \times 10^{-16} \pm 6 \%$ & $1.8 \times 10^{-2}$ & $2.1 \times 10^{-10}: 5 \tau$ & $3.0 \times 10^{-8}, 7^{\sigma}$ & $7.0 \times 10^{-3}$ \\
\hline & 12 & $1.0 \times 10^{-17}+17 \%$ & $8.2 \times 10^{-19}+47^{x}$ & $8.2 \times 10^{-2}$ & - & $5.2 \times 10^{-15}: 85 \%$ & $1.9 \times 10^{-2}$ & $3.4 \times 10^{-10}+3^{\sigma}$ & $4.9 \times 10^{-8}=4^{x}$ & $6.0 \times 10^{-3}$ \\
\hline & 13 & $1.4 \times 10^{-17}+11 \%$ & $1.3 \times 10^{-18}+30^{\sigma}$ & $9.3 \times 10^{-2}$ & - & $7.5 \times 10^{-16}+6^{r}$ & $1.9 \times 10^{-2}$ & $3.1 \times 10^{-10}: 3$ & $4.3 \times 10^{-8}, 4 m$ & $7.2 \times 10^{-33}$ \\
\hline & 14 & $1.6 \times 10^{-17}+10 \%$ & $1.3 \times 10^{-18}: 25 r$ & $8.1 \times 10^{-2}$ & $1.9 \times 10^{-98}+36 \%$ & $7.4 \times 10^{-16}: 6^{4}$ & $2.2 \times 10^{-2}$ & $1.9 \times 10^{-10} \cdot 3^{\sigma}$ & $2.14 \times 10^{-8}+4^{\pi}$ & fi. $13 \times 10^{-3}$ \\
\hline & 15 & $1.7 \times 10^{-17} \pm 10^{n !}$ & $2.3 \times 10^{-18}, 21^{n !}$ & $1.4 \times 10^{-1}$ & $1.7 \times 10^{-19} \pm 37 \omega_{n}$ & $B_{.} t \times 10^{-1 f_{1}}: 55_{0}$ & $2.1 \times 10^{-2}$ & $3.4 \times 10^{-10}: 3^{n}$ & $+.7 \times 10^{-8} \div 4^{\pi}$ & 6. $" \times 10^{-3}$ \\
\hline \multirow[t]{6}{*}{ Apr. } & 1 & $4.0 \times 10^{-17}+8 a_{0}$ & $3.0 \times 10^{-111}+20^{0}$ & $7.5 \times 10^{-2}$ & - & $9.1 \times 10^{-10}: 50^{\circ}$ & $4.4 \times 10^{-2}$ & $4.5 \times 10^{-20}: 45$ & $6.1 \times 11^{-3}: 5^{0}$ & $7.5 \times 10^{-3}$ \\
\hline & 2 & $1.8 \times 10^{-17}+11 \%$ & $3.0 \times 10^{-1 / 3}: 24^{0 .}$ & $1.7 \times 10^{-1}$ & - & $9.6 \times 10^{-16}: 6^{r}$ & $1.9 \times 10^{-2}$ & $3.7 \times 10^{-10}, 3^{7}$ & $5.0,10^{-8}, 4^{\sigma}$ & $7.4 \times 10^{-3}$ \\
\hline & 12 & $2.9 \times 10^{-17} \pm 80_{n}$ & $2.6 \times 10^{-1 \%}: 19 \%$ & $9.0 \times 10^{-2} 3$ & $3.4 \times 10^{-18}, 23^{6}$ & $1.3 \times 10^{-15}+6^{r_{0}}$ & $2.2 \times 10^{-2}$ & $5.2 \times 10^{-10}: 4 \div$ & $7.6 \times 10^{-8}: 36$ & $6 . n \times 16^{-3}$ \\
\hline & 13 & $2.3 \times 10^{-17}+8^{10}$ & $2.7 \times 10^{-1}: 17^{\pi}$ & $1.2 \times 10^{-1} \mathrm{l}$ & $1.6 \times 10^{-18}=22^{2}$ & $1.4 \times 10^{-15}+10^{0}$ & $1.6 \times 10^{-2}$ & $4.8 \times 10^{-10} \cdot 3^{7}$ & $6.7 \times 10^{-8}: 3^{x}$ & $7.2 \times 10^{-3}$ \\
\hline & 14 & $8.0 \times 10^{-14}+5^{0}$ & $2.3 \times 10^{-17} \div 7^{\circ}$ & $2.4 \times 10^{-4} \mathrm{l}$ & $1.6 \times 10^{-16}+8$ & $9.6 \times 10^{-16}+6$ & $8.3 \times 10^{-1}$ & $3.5 \times 10^{-10} \cdot 3^{m}$ & $5.0 \cdot 10^{-8} \cdot 3^{2}$ & $7.0 \times 10^{-3}$ \\
\hline & 15 & $2.5 \times 10^{-17}+7 \pi_{2}$ & $3.0 \times 10^{-18}=15^{\prime \prime}:$ & $1.2 \times 10^{-1}$ & $1.4 \times 10^{-18}: 21^{x}$ & $1.3 \times 10^{-15}, 0^{\pi}$ & $1.9 \times 10^{-2}$ & $5.5 \times 10^{-10} \cdot 2$ & $7, r \times 10^{-4} \cdot 3^{m}$ & $7.2 \times 10^{-3}$ \\
\hline \multirow[t]{6}{*}{ May } & 1 & $3.8 \times 10^{-17} \pm 11 \%$ & $2.4 \times 10^{-18} \pm 33^{\sigma}$ & $6.3 \times 10^{-2}$ & - & $1.4 \times 10^{-15}: 110$ & $2.7 \times 10^{-2}$ & $5.3 \times 10^{-10} 3^{r}$ & $7.0 \times 10^{-\gamma} \cdot 4$ & $7.6 \times 10^{-3}$ \\
\hline & 2 & $2.6 \times 10^{-17} \div 12^{2}$ & $3.8 \times 10^{-18}+25^{0}$ & $1 . \therefore \times 10^{-1}$ & - & $1.4 \times 10^{-15}=15 \%$ & $1.9 \times 10^{-2}$ & $5.0 \times 10^{-10} \cdot 3^{3}$ & $6.5 \times 10^{-8} \cdot 30$ & $7.6 \cdot 10^{-3}$ \\
\hline & 12 & $2.5 \times 10^{-17} \pm 8 \%$ & $2.4 \times 10^{-18} \div 170$ & $1.0 \times 10^{-1}$ & - & i. $6 \times 10^{-15}: 21 \pi$ & $1.5 \times 10^{-2}$ & $0.3,\left[0^{-10}+3^{5}\right.$ & $15.7,10^{-13}, 35$ & $7.211^{-9}$ \\
\hline & 13 & $2.8 \times 10^{-17}: 8 \%$ & $2.6 \times 10^{-18} \div 18^{m}$ & $0.3 \times 10^{-2}$ & - & $1.5 \times 10^{-15}: 15^{T_{1}}$ & $1.9 \times 10^{-2}$ & $5.1 \times 10^{-10}: 3$ & fi. $6 \times 10^{-1} \cdot 3^{+\infty}$ & $i .7 \times 10^{-1}$ \\
\hline & $1+$ & $4.4 \times 10^{-15} \pm 5 \sigma_{10}$ & $1.2 \times 10^{-16} \pm 6^{\circ}$ & $2.7 \times 10^{-2} 8$ & $8.8 \times 10^{-10}: 7 \%$ & $8.0 \times 10^{-16} \div 7^{r}$ & 5.5 & $4.1 \times 10^{-10}: 3$ & $5.4 \times 10^{-8}, 7$ & $i, f, 10^{-\ell}$ \\
\hline & 15 & $1.7 \times 10^{-17} \pm 10 \%$ & $1.6 \times 10^{-18} \pm 28^{m}$ & $3.4 \times 10^{-2}$ & - & $1.1 \times 10^{-15}=12^{5}$ & $1.5 \times 10^{-2}$ & $5.8 \times 10^{-10} \cdot 3^{x}$ & $7.7 \times 10^{-3} \cdot 7 \mathrm{~m}$ & $7,5 \times 10^{\circ}$ \\
\hline \multirow[t]{4}{*}{ June } & 1 & $1.1 \times 10^{-17}+15 \%$ & $4.5 \times 10^{-10}=42 \%$ & $4.1 \times 10^{-2}$ & - & $5.4 \times 10^{-16} \div 23^{\gamma}$ & $2.0 \times 10^{-2}$ & $5.3 \times 10^{-10} \cdot 3^{5}$ & $i . H, 10^{-3},+$ & $7.5 \times 10^{.9}$ \\
\hline & 2 & $1.2 \times 10^{-17} \ddagger 15 \%$ & $1.5 \times 10^{-18}+345$ & $1.3 \times 10^{-1}$ & - & $1.0 \times 10^{-15}+41 \%$ & $1.2 \times 10^{-2}$ & $6.2 \times 10^{-10}: 3^{m}$ & $\because 3 \times 10^{-4}: 3=$ & $8.3 \times 10^{-3}$ \\
\hline & 12 & $5.3 \times 10^{-17} \pm 7 \%$ & $3.3 \times 10^{-18} \cdot 19^{\tau}$ & $6.2 \times 10^{-2}$ & - & $2.0 \times 10^{-15}+22 c_{0}$ & $2.7 \times 10^{-2}$ & $7.0 \times 10^{-10} \div 3$ & $9.6 \times 10^{-i}: 3^{n}$ & $7.1 \times 10^{-3}$ \\
\hline & 13 & $1.2 \times 10^{-17} \pm 970$ & $1.8 \times 10^{-18}: 20^{10}$ & $1.5 \times 10^{-1}$ & - & $8.8 \times 10^{-16}+30 \%$ & $1.4 \times 10^{-2}$ & $7.1 \times 15^{-10}: 3$ & $4.6 \times 10^{-3}: 3 n^{\circ}$ & $7.4 \times 10^{-3}$ \\
\hline
\end{tabular}


Table 3 (continued).

\begin{tabular}{|c|c|c|c|c|c|c|c|c|c|c|}
\hline \multirow[b]{2}{*}{ Month } & \multirow[b]{2}{*}{ Lacation } & \multirow{2}{*}{${ }^{239} \mathrm{Pu}$} & \multirow[b]{2}{*}{${ }^{238} \mathrm{Pu}$} & \multirow[b]{2}{*}{${ }^{238} \mathrm{Pu} /{ }^{239} \mathrm{Pu}$} & \multirow[b]{2}{*}{$241 \mathrm{Am}$} & \multirow[b]{2}{*}{${ }^{90} \mathrm{Sr}$} & \multirow[b]{2}{*}{${ }^{239} \mathrm{Pu} /{ }^{90} \mathrm{Sr}$} & \multicolumn{2}{|c|}{ Mass $\left(\mathrm{m} / / \mathrm{M}^{3}\right)$} & \multirow[b]{2}{*}{$235_{V}{ }^{238} \mathrm{C}$} \\
\hline & & & & & & & & $235^{(!)}$ & $23 \mathrm{U}$ & \\
\hline \multirow{2}{*}{$\begin{array}{l}\text { Junc } \\
\text { (con'd) }\end{array}$} & 14 & $1.3 \times 10^{-15} \pm 5 \%$ & $4.2 \times 10^{-17} \pm 7 \%$ & $3.2 \times 10^{-2}$ & - & $3.7 \times 10^{-16} \div 9 \%$ & 3.5 & $5.7 \times 10^{-10} \div 3 \%$ & $7.5 \times 10^{-8}+3 \%$ & $7.6 \times 10^{-3}$ \\
\hline & 15 & $1.7 \times 10^{-17} \pm 10 \%$ & $1.7 \times 10^{-18} \pm 26 \%$ & $1.0 \times 10^{-1}$ & - & $8.5 \times 10^{-16}=18 \%$ & $2.0 \times 10^{-2}$ & $7.4 \times 10^{-10} \pm 3 \%$ & $1.0 \times 10^{-7}: 3 \%$ & $7.4 \times 10^{-3}$ \\
\hline \multirow[t]{6}{*}{ July } & 1 & $1.1 \times 10^{-17}+14 \%$ & $1.5 \times 10^{-18} \pm 34 \%$ & $1.4 \times 10^{-1}$ & - & $5.7 \times 10^{-16} \pm 12^{\circ}$ & $1.0 \times 10^{-2}$ & $3.7 \times 10^{-10} \pm 3$ & $5.2 \times 10^{-8}+45$ & $7.1 \times 10^{-3}$ \\
\hline & 2 & $1.3 \times . J^{-17}+30 \%$ & $6.4 \times 10^{-18}+33 \%$ & $4.9 \times 10^{-1}$ & - & $1.3 \times 10^{-15}+18^{\circ}$ & $1.0 \times 10^{-2}$ & $9.8 \times 10^{-10}+3 \%$ & $1.3 \times 10^{-7} \div 45$ & $7.5 \times 10^{-3}$ \\
\hline & 12 & $1.1 \times 10^{-17}+36 \%$ & $2.4 \times 10^{-18}+46 \%$ & $2.2 \times 10^{-1}$ & - & $6.6 \times 10^{-16} \pm 13 \%$ & $1.7 \times 10^{-2}$ & $5.5 \times 10^{-31}+2 \%$ & $8.1 \times 10^{-8}=45$ & $6.8 \times 10^{-3}$ \\
\hline & 13 & $8.2 \times 10^{-18} \pm 12 \%$ & $1.7 \times 10^{-18}+26 \%$ & $2.1 \times 10^{-1}$ & - & $5.2 \times 10^{-16}=14 \%$ & $1.6 \times 10^{-2}$ & $4.7 \times 10^{-30} \pm 2 \%$ & $6.7 \times 10^{-8} \div 3 \%$ & $7.0 \times 10^{-3}$ \\
\hline & 14 & $1.1 \times 10^{-16} \pm 6 \%$ & $4.6 \times 10^{-18}+14 \%$ & $4.2 \times 10^{-2}$ & - & $6.2 \times 10^{-16} \div 12 \%$ & 1. $8 \times 10^{-1}$ & $5.9 \times 10^{-10}+2 \%$ & $8.2 \times 10^{-8}=3 \%$ & $7.2 \times 10^{-3}$ \\
\hline & 15 & $1.3 \times 10^{-17} \pm 14 \%$ & $6.2 \times 10^{-19} \pm 42 \%$ & $4.8 \times 10^{-2}$ & - & $3.0 \times 10^{-16} \pm 15 \%$ & $4.3 \times 10^{-2}$ & $1.4 \times 10^{-9} \pm 2 \%$ & $2.1 \times 10^{-7}+3 \%$ & $6.7 \times 10^{-3}$ \\
\hline \multirow[t]{6}{*}{ Aug. } & 1 & $6.7 \times 10^{-18} \pm 18 \%$ & $5.7 \times 10^{-19} \pm 52 \%$ & $8.5 \times 10^{-3}$ & - & $5.9 \times 10^{-16} \pm 16 \%$ & $1.1 \times 10^{-2}$ & $7.5 \times 10^{-10} \pm 4 \%$ & $9.9 \times 10^{-8}+5 \%$ & $7.6 \times 10^{-3}$ \\
\hline & 2 & $6.7 \times 10^{-18} \pm 17 \%$ & $7.5 \times 10^{-19} \pm 35 \%$ & $1.1 \times 10^{-1}$ & - & $6.8 \times: \tilde{u}^{-16} \pm 16 \%$ & $9.9 \times 10^{-3}$ & $6.4 \times 10^{-10} \pm 3 \%$ & $8.9 \times 10^{-8} \pm 4 \%$ & $7.2 \times 10^{-3}$ \\
\hline & 12 & $9.2 \times 10^{-18} \pm 16 \%$ & $1.1 \times 10^{-18} \pm 50 \%$ & $1.2 \times 10^{-1}$ & - & $7.5 \times 10^{-16}: 13 \%$ & $1.2 \times 10^{-2}$ & $6.2 \times 10^{-10} \pm 3 \%$ & $1.1 \times 10^{-7} \times 3 \%$ & $7.5 \times 10^{-3}$ \\
\hline & 1.3 & $1.0 \times 10^{-17} \pm 19 \%$ & $1.4 \times 10^{-18} 139 \%$ & $1.4 \times 10^{-1}$ & - & $6.7 \times 10^{-16} \pm 24 \%$ & $1.5 \times 10^{-2}$ & $8.2 \times 10^{-10} \pm 3 \%$ & $1.1 \times 10^{-7}+35$ & $7.5 \times 10^{-3}$ \\
\hline & 14 & $2.8 \times 10^{-16}+5 \%$ & $1.2 \times 10^{-17} \pm 11 \%$ & $4.3 \times 10^{-2}$ & - & $2.7 \times 10^{-16} \pm 9 \%$ & $9.6 \times 10^{-1}$ & $1.9 \times 10^{-9}: 3 \%$ & $2.7 \times 10^{-7}: 4 \%$ & $7.0 \times 10^{-3}$ \\
\hline & 15 & $7.7 \times 10^{-18}+13 \%$ & $8.6 \times 10^{-19} \pm 31 \%$ & $1.2 \times 10^{-1}$ & - & $5.8 \times 10^{-16} \pm 12 \%$ & $1.3 \times 10^{-2}$ & $1.2 \times 10^{-9} \pm 3 \%$ & $1.6 \times 10^{-7}+45$ & $7.5 \times 10^{-3}$ \\
\hline \multirow[t]{6}{*}{ Sept. } & 1 & $5.3 \times 10^{-18} \pm 15 \%$ & $9.2 \times 10^{-19} \neq 33 \%$ & $1.7 \times 10^{-1}$ & - & $2.7 \times 10^{-16} \pm 26 \%$ & $2.0 \times 10^{-2}$ & $4.9 \times 10^{-10} \div 3 \div$ & $7.0 \times 10^{-8}: 4$. & $7.0 \times 10^{-3}$ \\
\hline & 2 & $4.6 \times 10^{-18} \pm 16 \%$ & $1.5 \times 10^{-18} \pm 22^{\circ}$ & $3.3 \times 10^{-1}$ & - & $4.8 \times 10^{-16} \pm 22 \%$ & $9.6 \times 10^{-3}$ & $6.4 \times 10^{-10} \pm 3 \%$ & $8.6 \times 10^{-8} \pm 4 \%$ & $7.4 \times 10^{-3}$ \\
\hline & 12 & $2.2 \times 10^{-17} \pm 8 \%$ & $9.4 \times 10^{-19}+28 \%$ & $4.3 \times 10^{-2}$ & - & Lost & & $6.2 \times 10^{-10} \pm 37$ & $9.0 \times 10^{-8} \pm 4$ & $6.9 \times 10^{-3}$ \\
\hline & 13 & $3.5 \times 10^{-18}+19 \%$ & $1.2 \times 10^{-19} \pm 44^{\%}$ & $3.4 \times 10^{-2}$ & - & $3.6 \times 10^{-16} \pm 28 \%$ & $9.7 \times 10^{-3}$ & $4.6 \times 10^{-20} \pm 45$ & $6.2 \times 10^{-8} \pm 4 \%$ & $7.3 \times 10^{-3}$ \\
\hline & 14 & $5.8 \times 10^{-17} \pm 7 \%$ & $3.5 \times 10^{-18}=17 \%$ & $6.0 \times 10^{-2}$ & - & $4.5 \times 10^{-16} \neq 279$ & $1.3 \times 10^{-1}$ & $5.7 \times 10^{-10} \neq 4 \%$ & $8.2 \times 10^{-8}=47$ & $7.0 \times 10^{-3}$ \\
\hline & 15 & $4.8 \times 10^{-18} \pm 14 \%$ & $6.8 \times 10^{-19} \pm 32 \%$ & $1.4 \times 10^{-1}$ & - & $4.8 \times 10^{-16} \pm 26 \%$ & $1.0 \times 10^{-2}$ & $8.6 \times 10^{-10} \pm 3 \%$ & $1.2 \times 10^{-7}+40$ & $7.2 \times 10^{-3}$ \\
\hline \multirow[t]{6}{*}{ Oct. } & 1 & $6.8 \times 10^{-18} \pm 21 \%$ & $1.0 \times 10^{-18}+48^{6 \%}$ & $1.5 \times 10^{-1}$ & - & $7.0 \times 10^{-16}+17 \%$ & $0.7 \times 10^{-3}$ & $4.6 \times 10^{-10}=3 \tau_{7}$ & $7,1 \times 10^{-8}+15$ & $6.5 \times 10^{-3}$ \\
\hline & 2 & $5.3 \times 10^{-18} \pm 22 \%$ & - & - & - & $9.2 \times 10^{-16} \pm 12 \%$ & $5.8 \times 10^{-3}$ & $6.6 \times 10^{-10} \pm 3^{5}$ & $1.7 \times 10^{-7}+45$ & $3.9 \times 10^{-3}$ \\
\hline & 12 & $1.6 \times 10^{-17} \pm 12 \%$ & $8.5 \times 10^{-19} \pm 41 \%$ & $5.3 \times 10^{-2}$ & - & $6.9 \times 10^{-16} \pm 20 \%$ & $2.3 \times 10^{-2}$ & $1.0 \times 10^{-9}=3 \%$ & $2.6 \times 10^{-7}+45$ & $3.0 \times 10^{-3}$ \\
\hline & 13 & $5.6 \times 10^{-18}+16 \%$ & $6.5 \times 10^{-19}+37 \%$ & $1.2 \times 10^{-1}$ & - & $5.7 \times 10^{-16} \pm 23 \%$ & $9.8 \times 10^{-3}$ & $5.9 \times 10^{-10}=3^{r}$ & $1.2 \times 10^{-7} \div 4 \%$ & $4.3 \times 10^{-3}$ \\
\hline & 14 & $4.3 \times 10^{-18}+10 \%$ & $3.5 \times 10^{-18}+35$ & $3.5 \times 10^{-1}$ & - & $4.5 \times 10^{-16}, 29 \%$ & $9.9 \times 10^{-3}$ & $9.2 \times 10^{-10}=3 \%$ & $1.6 \times 10^{-7}=49$ & $5.7 \times 10^{-3}$ \\
\hline & 15 & $1.6 \times 10^{-17}+125$ & $9.6 \times 10^{-19}+32 r$ & $6.0 \times 10^{-2}$ & - & $5.9 \times 10^{-16}=21 \%$ & $2.7 \times 10^{-2}$ & $9.3 \times 10^{-10}=3 \%$ & $1.6 \times 10^{-7} \div 90$ & $5.8 \times 10^{-3}$ \\
\hline \multirow[t]{6}{*}{ Nov. } & 1 & $2.1 \times 10^{-18}+229 \%$ & $4.2 \times 10^{-19} \pm 49 \%$ & $2.0 \times 10^{-1}$ & - & $3.3 \times 10^{-16}=28 \%$ & $6.4 \times 10^{-3}$ & $1.7 \times 10^{-10}=4 \%$ & $2.1 \times 10^{-8}=6 \%$ & $8.1 \times 10^{-3}$ \\
\hline & 2 & $2.3 \times 10^{-18} \neq 30 \%$ & $6.8 \times 10^{-20}+100 \%$ & $2.6 \times 10^{-2}$ & - & $6.4 \times 10^{-16}+167$ & $3.6 \times 10^{-3}$ & $1.9 \times 10^{-10}=5 \%$ & $2.7 \times 10^{-8} \leq 7 \%$ & $7.0 \times 10^{-3}$ \\
\hline & 12 & $3.4 \times 10^{-18} \pm 28 \%$ & $4.8 \times 10^{-19} \pm 61 \%$ & $1.4 \times 10^{-1}$ & - & $6.1 \times 10^{-16}: 218$ & $5.6 \times 10^{-3}$ & $2.6 \times 10^{-10}=4 \%$ & $3.9 \times 10^{-8} \pm 5 \%$ & $6.7 \times 10^{-3}$ \\
\hline & 13 & $2.7 \times 10^{-18}+31 \%$ & $1.5 \times 10^{-19} \pm 76 \%$ & $5.6 \times 10^{-2}$ & - & $5.2 \times 10^{-16} \neq 175$ & $5.2 \times 10^{-3}$ & $2.2 \times 10^{-10}=7 \%$ & $3.1 \times 10^{-8}=10 \%$ & $7.1 \times 10^{-3}$ \\
\hline & 14 & $6.7 \times 10^{-17} \pm 8 \%$ & $2.2 \times 10^{-1 B} \pm 24 \%$ & $3.3 \times 10^{-2}$ & - & $5.3 \times 10^{-16} \pm 187$ & $1.3 \times 10^{-1}$ & $1.9 \times 10^{-10} \div 57$ & $2.7 \times 10^{-8} \div 75$ & $7.0 \times 10^{-3}$ \\
\hline & 15 & $2.2 \times 10^{-18}+22 \%$ & $2.9 \times 10^{-19} \pm 41 \%$ & $1.3 \times 10^{-1}$ & - & $3.7 \times 10^{-26} \pm 23 \%$ & $5.9 \times 10^{-3}$ & $2.6 \times 10^{-10} \div 45$ & $5.0 \times 10^{-8}=6 \%$ & $5.2 \times 10^{-3}$ \\
\hline \multirow[t]{2}{*}{ nec. } & 1 & $3.4 \times 10^{-18} \pm 22 \%$ & $6.4 \times 10^{-19} \pm 45 \%$ & $1.9 \times 10^{-1}$ & - & $8.1 \times 10^{-26} \pm 16 \%$ & $4.2 \times 10^{-3}$ & $3.2 \times 10^{-10} \pm 5 \%$ & $1.1 \times 10^{-7}=67$ & $2.0 \times 10^{-3}$ \\
\hline & 2 & $4.1 \times 10^{-18} \pm 28 \%$ & $4.5 \times 10^{-19} \pm 64 \%$ & $1.1 \times 10^{-1}$ & - & $8.2 \times 10^{-16} \pm 227$ & $5,0 \times 10^{-3}$ & $8.0 \times 10^{-10}=4 \%$ & $3.5 \times 10^{-7}=57$ & $2.3 \times 10^{-3}$ \\
\hline
\end{tabular}


Table 3 (continued).

\begin{tabular}{|c|c|c|c|c|c|c|c|c|c|c|}
\hline \multirow[b]{2}{*}{ Month } & \multirow[b]{2}{*}{ Location } & \multirow[b]{2}{*}{${ }^{239} \mathrm{Pu}$} & \multirow[b]{2}{*}{${ }^{238} \mathrm{Pu}$} & \multirow[b]{2}{*}{${ }^{238} \mathrm{Pu} j^{239} \mathrm{Pu}$} & \multirow[b]{2}{*}{$241 \mathrm{Am}$} & \multirow[b]{2}{*}{$3_{3 r}^{30}$} & \multirow[b]{2}{*}{${ }^{239} \mathrm{Pe}{ }^{90} \mathrm{Sr}$} & \multicolumn{2}{|c|}{ Huss $\left(\mathrm{mg}, \mathrm{m}^{3}\right)$} & \multirow[b]{2}{*}{$235_{\mathrm{r}} .239 \mathrm{~s}_{\mathrm{T}}$} \\
\hline & & & & & & & & $235 !$ & $23 \% 1$ & \\
\hline \multirow{4}{*}{$\begin{array}{l}\text { Dec. } \\
\text { (cont' } d\}\end{array}$} & 12 & $5.2 \times 10^{-18}+26 \%$ & $1.9 \times 10^{-19} \pm 71 \%$ & $3.7 \times 10^{-2}$ & - & $7.2 \times 10^{-16} \pm 21^{\circ}$ & $7.2 \times 10^{-3}$ & $4.1 \times 10^{-10}=5$ & $1.1 \times 10^{-i}=9 \%$ & $3.7 \times 10^{-3}$ \\
\hline & 13 & $4.0 \times 10^{-18}+31 \%$ & $3.4 \times 10^{-19}+71 \%$ & $8.5 \times 10^{-2}$ & - & $8.6 \times 10^{-16} \pm 2: *$ & $i .7 \times 10^{-3}$ & $1.8 \times 10^{-10}: 7 \%$ & $2 . \therefore \times 10^{-B}=9 \%$ & $6.2 \times 15^{-3}$ \\
\hline & 14 & $5.8 \times 10^{-18} \pm 16 \%$ & $3.7 \times 10^{-19}+42 \%$ & $6.4 \times 10^{-2}$ & - & $1.0 \times 10^{-15} \neq 16 \%$ & $5.8 \times 10^{-3}$ & $7.0 \times 10^{-10}: .5$ & $3.1 \times 10^{-7}=60$ & $2.3 \times 10^{-3}$ \\
\hline & 25 & $3.4 \times 10^{-18} \pm 26 \%$ & $1.9 \times 10^{-19}+64 \%$ & $5.6 \times 10^{-2}$ & - & $7.2 \times 10^{-16} ; 17^{\pi}$ & $4.7 \times 10^{-3}$ & $3.8 \times 10^{-10} \cdot 4^{10}$ & $1.5 \times 10^{-7} \div 5$ & $2 .^{-} \times 10^{-3}$ \\
\hline
\end{tabular}

Annual averages

Locaticn ${ }^{239} \mathrm{Pu} \mu \mathrm{Ci} / \mathrm{ml}$ of $\mathrm{RCG}^{\mathrm{a}} \quad{ }^{238} \mathrm{Pu} \mu \mathrm{Ci} / \pi 1 \%$ \% $\mathrm{RCG}$

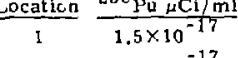

2

12

13

14

15
$1.1 \times 10^{-17}$

$1.7 \times 10^{-17}$

$1.1 \times 10^{-17}$

$7.2 \times 10^{-15}$

$1.3 \times 10^{-17}$

\section{$1.5 \times 10^{-3}$}

$1.1 \times 10^{-3}$

$1.7 \times 10^{-3}$

$1.1 \times 10^{-3}$

$7.2 \times 10^{-1}$

$1.3 \times 10^{-3}$
$1.4 \times 10^{-18}-1.4 \times 10^{-4}$

$<1.5 \times 10^{-18}$

$1.6 \times 10^{-1}$

$1.3 \times 10^{-18}$

$1.9 \times 10^{-17}$

$1.2 \times 10^{-18}$
$-1,5 \times 10^{-4}$

$1.6 \times 10^{-4}$
$1.3 \times 10^{-4}$

$1.9 \times 10^{-3}$

$1.2 \times 10^{-4}$ $\frac{235 \mathrm{u}, \mathrm{mg} / \mathrm{m}^{3}}{4.1 \times 10^{-10}}$

$5.3 \times 10^{-10}$

$5 .+\times 10^{-10}$

$4.4 \times 10^{-10}$

$5.9 \times 10^{-10}$

$5.6 \times 10^{-10}$ $\frac{\% \mathrm{RCG}}{2 . \times 10^{-5}}$

$2.8 \times 10^{-5}$

$2.8 \times 10^{-5}$

$2.3 \times 10^{-5}$

$3.1 \times 10^{-5}$

$2.9 \times 10^{-5}$ $\frac{238 \mathrm{t}, \mathrm{mg} / \mathrm{m}^{3}}{63 \times 10^{-8}}=\frac{\mathrm{rgG}}{2 \times 10^{-4}}$

$3.9 \times 10^{-8} \quad 6.6 \times 10^{-4}$

$9.2 \times 10^{-8} \quad 6.1 \times 10^{-4}$

$6.4 \times 10^{-8} \quad 4.3 \times 10^{-4}$

$1.0 \times 10^{-7} \quad 6.7 \times 10^{-4}$

$1.0 \times 10^{-7} \quad 6.7 \times 10^{-4}$

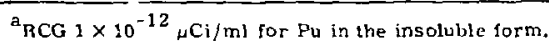

Table 4. Tritium (HTO) in air at Laboratory perimeters in $1973(\mu \mathrm{Ci} / \mathrm{ml})$.

\begin{tabular}{|c|c|c|c|c|c|c|c|c|c|c|c|}
\hline \multirow{2}{*}{$\begin{array}{l}\operatorname{los} \operatorname{lat}^{-} \\
\operatorname{lin}^{3}\end{array}$} & \multirow{2}{*}{$\begin{array}{l}\text { No. } \\
\text { of } \\
\text { sitmples }\end{array}$} & \multicolumn{3}{|c|}{ January-.lun. } & \multirow{2}{*}{$\begin{array}{l}\text { Vu. } \\
\text { sil } \\
\text { samplem }\end{array}$} & \multicolumn{3}{|c|}{ July-tracember } & \multirow{2}{*}{$\begin{array}{l}\text { Int:1 } \\
1 \ldots+1 .\end{array}$} & \multirow[b]{2}{*}{$1: c_{i}^{b}$} & \multirow{2}{*}{ 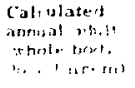 } \\
\hline & & Maximum & Minimini & Werab" & & Maximum & :l 1 лı rmism & 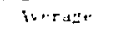 & & & \\
\hline 1 & 2 & $7.0 \times 10^{-12} \cdot 30.37^{\circ}$ & $1,5 \times 10^{-12} \cdot 3.1^{0}$ & $1 \ldots \times 10^{-12}$ & 23 & $1.7 \times 10^{-11} \cdot 3.2^{7}$ & $0.7 \times 1+12: 25.7$ & $3.0 \times 10^{-11}$ & $2.4+10 !$ & $1 .+10^{2}$ & $4.1 \mathrm{H}^{-1}$ \\
\hline 2 & 0 & & & & 10 & $4.0 \times 10^{-11}: 3.2$ & $1.7 \times 10^{-11} \cdot 9.17$ & $1 . ; \times 10^{-11}$ & $4.10)^{11}$ & $1.2,102$ & $5.1,+10$ \\
\hline 12 & 0 & & & & 15 & $1.1110^{-10} \cdot 1,5^{-}$ & $2.7 \cdot 10^{-12} \quad 14.25$ & $6.2 \times 10^{-11}$ & $6.2 \times 10^{11}$ & $\therefore 1 \cdot 10^{2}$ & $\because 111^{2}$ \\
\hline 1.3 & 0 & & & & 12 & $4.0 \times 10^{-11} \cdot 4.35$ & $8,6,10^{0}+2 \quad 15.0^{\circ}$ & $2.1 \times 10^{-11}$ & $2.4 \times 10^{-11}$ & $1,2 \cdot 102$ & 3. $,: 11: ?$ \\
\hline 14 & 3 & $3.7 \times 10^{-11} \cdot 4.0^{r}$ & $1.4 \times 10^{-11}=6.7 \%$ & $2.6<10^{-11}$ & 23 & $1.2 \times 10^{-10} \cdot 2.5 \%$ & 3. $\times 10^{-11}: 8.37$ & $6.1 \times 10^{-11}$ & $5,2 \times 10^{11}$ & $1 . n^{2}$ & $\therefore(t+1)$ \\
\hline 15 & 3 & $1.2 \times 10^{-11}=4.7^{\circ}$ & $7.5 \times 10^{-12}: 10.4^{.}$ & $1.0 \times 10^{-11}$ & 20 & $4.8 \cdot 10^{-11} \cdot 4.4^{-}$ & $5.4 \times 10^{-12} \cdot 46.8$ & $2.1 \times 10^{-11}$ & $2.0 \times 11^{-11}$ & $\because 10^{\circ}$ & $\therefore 2,10)^{?}$ \\
\hline
\end{tabular}

a see fig. 2 for locations.

here for 13 ro in air $2 \times 10^{-i} \mu\left(\mathrm{C}_{1}, \mathrm{ml}\right.$.

"Aased on fuly-lecember daia. 
Table 5. Activity levels of various radionuclides observed in hiverm

\begin{tabular}{|c|c|c|c|c|c|c|}
\hline Location ${ }^{a}$ & $239_{\mathrm{Pu}}$ & $238_{\mathrm{Pu}}$ & ${ }^{137} \mathrm{Cs}_{5}$ & ${ }^{214} \mathrm{Bi}\left(\mathrm{C}^{\prime}\right)$ & $212 \mathrm{~Pb}(\mathrm{Th})$ & ${ }^{4} 0_{K}$ \\
\hline $5-253$ & $6.3 \times 10^{-9} \pm 6 \%$ & $3.7 \times 10^{-10} \pm 16 \%$ & $3.5 \times 10^{-7} \pm 14^{\prime \prime \prime}$ & $6.1 \times 10^{-7} \pm 8^{\pi}$ & $6.0 \times 10^{-7}: 0^{r}$ & $0.8 \times 10^{-6} \cdot n$ \\
\hline$S-254$ & $6.7 \times 10^{-8}+6$ & $7.7 \times 10^{-10} \pm 8^{0 \%}$ & $4.8 \times 10^{-7} \div 12 \%$ & $5.3 \times 10^{-7} \pm 10 \%$ & $5.3 \times 10^{-7}=110:$ & $9.1 \times 10^{-6}+99^{\pi}$ \\
\hline$S-255$ & $4.6 \times 10^{-9} \pm 6 \%$ & $5.0 \times 10^{-10} \pm 14 \%$ & $1.7 \times 10^{-7} \pm 22^{\%}$ & $4.3 \times 10^{-7}=8$ & $5.0 \times 10^{-7} \div 80$ & $3.6 \times 10^{-6} \div 8$ \\
\hline$S-256$ & $4.1 \times 10^{-9}+9 \%$ & $2.6 \times 10^{-10}+26^{\%}$ & $1.9 \times 10^{-7} \pm 24 \%$ & $6.3 \times 10^{-7} \pm 9 \%$ & $6.2 \times 10^{-7}=0 \%$ & $1.2 \times 10^{-5}: 33^{n}$ \\
\hline$S-257$ & $4.3 \times 10^{-9}+7 \%$ & $1.8 \times 10^{-10}+21 \%$ & $2.2 \times 10^{-7} \pm 18 \%$ & $6.0 \times 10^{-7} \pm 8 \%$ & $6.3 \times 10^{-7}: 90^{0}$ & $1.1 \times 10^{-5}: 8 \pi$ \\
\hline$S-259$ & $3.9 \times 10^{-9} \pm 7 \%$ & $2.2 \times 10^{-10} \pm 19 \%$ & $2.3 \times 10^{-7} \pm 19 \sigma_{n}$ & $5.6 \times 10^{-7} \div 8 \%$ & $5.0 \times 10^{-7}+9 r$ & $1.3 \times 10^{-5} \div 7 \%$ \\
\hline$S-264$ & $2.3 \times 10^{-9}+11 \%$ & $1.4 \times 10^{-10}+30 \%$ & $1.2 \times 10^{-7} \pm 34 \%$ & $6.2 \times 10^{-7} \pm 7$ & $7.2 \times 10^{-7} \leq 7$ & $1.2 \times 10^{-5} \div 8 \%$ \\
\hline S-267 & $3.7 \times 10^{-9} \pm 9 \%$ & $2.2 \times 10^{-10}+13^{\%}$ & $2.4 \times 10^{-7} \pm 19 \%$ & $5.8 \times 10^{-7} \pm 8^{\sigma}$ & $6.6 \times 10^{-7}=8 \pi^{\pi}$ & $1.1 \times 10^{-5}: 5^{n}$ \\
\hline$S-268$ & $3.4 \times 10^{-9}+8 \%$ & $2.7 \times 10^{-10} \pm 24 \%$ & $2.2 \times 10^{-7} \pm 20 \%$ & $5.7 \times 10^{-7} \pm 8 \%$ & $5.9 \times 10^{-7} \div 9 \%$ & $1.3 \times 10^{-5}: 4 n^{m}$ \\
\hline$S-271$ & $9.5 \times 10^{-9} \pm 7 \%$ & $4.4 \times 10^{-10} \pm 20 \%$ & $5.1 \times 10^{-7} \div 10 \%$ & $6.1 \times 10^{-7} \div 7 \%$ & $6.7 \times 10^{-7}: 7 \%$ & $1.4 \cdot 10^{-5} \div 6 \%$ \\
\hline$S-272$ & $5.4 \times 10^{-9} \pm 10 \%$ & $7.7 \times 10^{-10}: 20 \%$ & $2.0 \times 10^{-7}: 20 \%$ & $6.0 \times 10^{-7}+7 \%$ & $7.3 \times 10^{-7} \div 7 \%$ & $1.3 \times 10^{-5}: 7 \%$ \\
\hline$S-275$ & $7.7 \times 10^{-9}+11 \%$ & $5.1 \times 10^{-10}: 32^{m}$ & $4.6 \times 10^{-7}+11 \%$ & $5.0 \times 10^{-7} \pm 8 \pi_{2}$ & $6.2 \times 10^{-T}=9 \tau$ & $0.2 \times 10^{-6} \pm 3^{r_{n}}$ \\
\hline$S-277$ & $3.9 \times 10^{-9}+27 \%$ & $3.2 \times 10^{-10} \pm 27 \%$ & $1.8 \times 10^{-7} \pm 18^{\sigma}$ & $4.1 \times 10^{-7} \pm 9$ & $5.1 \times 10^{-7}=9 \%$ & $5.9 \times 10^{-6}: 10^{2}$ \\
\hline$S-278$ & $4.1 \times 10^{-9} \pm 12 \%$ & $1.7 \times 10^{-10} \pm 380$ & $3.5 \times 10^{-7} \div 12^{c}$ & $3.5 \times 10^{-7} \pm 11 \%$ & $4.4 \times 10^{-7}+11 \%$ & $6.4 \times 10^{-6}=10^{2}$ \\
\hline $5-283$ & $2.6 \times 10^{-8} \div 6 \%$ & $1.8 \times 10^{-9}+10^{m}$ & $3.0 \times 10^{-7}+15 \%$ & $6.6 \times 10^{-7} \div 6^{\sigma i n}$ & $8.0 \times 10^{-7}+7 m_{n}$ & $1.2 \times 10^{-5}:-0^{\circ}$ \\
\hline$S-284$ & $2.8 \times 10^{-5}+9 \%$ & $1.7 \times 10^{-10} \pm 29 \%$ & $6.9 \times 10^{-8}: 50 \%$ & $4.7 \times 10^{-7}+9$ & $6.1 \times 1 \nu^{-7}+9 m_{0}$ & $7.9 \times 10^{-6} \div 9$ \\
\hline$S-285$ & $9.0 \times 10^{-9}+7 \%$ & $9.8 \times 10^{-10}+13 \%$ & $4.2 \times 10^{-7} \pm 10 \%$ & $3.8 \times 10^{-7} \pm 9 \%$ & $4.9 \times 10^{-7}=9 \%$ & $9.1 \times 10^{-6}=9^{n}$ \\
\hline$s-286$ & $2.7 \times 10^{-9}+11 \%$ & $2.2 \times 10^{-10} \pm 28^{m}$ & $1.5 \times 10^{-7}+24 \%$ & $6.5 \times 10^{-7}=8 \%$ & $7.2 \times 10^{-7} \div 9 \%$ & $1.2 \times 10^{-5}: 7 \cdots$ \\
\hline$S-287$ & $2.0 \times 10^{-8}+6 \%$ & $1.0 \times 10^{-9} \pm 14^{0 !}$ & $2.4 \times 10^{-7}+17 \%$ & $5.4 \times 10^{-7} \pm 12 \%$ & $5.4 \times 10^{-7}=13^{m}$ & $1.2 \times 10^{-5}+10^{\circ}$ \\
\hline$S-288$ & $4.0 \times 10^{-9}+10 \%$ & $3.5 \times 10^{-10}+27 \%$ & $2.2 \times 10^{-7}+18 \sigma_{0}$ & $6.4 \times 10^{-7} \pm 11$ & $6.1 \times 10^{-7}=11 \%$ & $1.2 \times 10^{-5}=7$ \\
\hline$S-289$ & $8.2 \times 10^{-9}+8 \%$ & $5.6 \times 10^{-10}+21 \%$ & $4.1 \times 10^{-7}=12 \%$ & $5.9 \times 10^{-7} \pm 9^{m}$ & $7.2 \times 10^{-7}: n^{r}$ & $1.2 \times 10^{-5}=7^{n}$ \\
\hline$S-290$ & $9.3 \times 10^{-9}+9 \%$ & $4.8 \times 10^{-10}+24 \%$ & $4.1 \times 10^{-7} \pm 8 \sigma_{7}$ & $6.1 \times 10^{-7} \pm 6 \%$ & $6.2 \times 10^{-7}=8 \%$ & $1.2 \times 10^{-5} \pm 5^{5}$ \\
\hline$S-292$ & $1.0 \times 10^{-8} \pm 8 \%$ & $5.5 \times 10^{-10} \pm 21 \%$ & $4.7 \times 10^{-7} \cdot 11^{0 .}$ & $5.5 \times 10^{-7}=3 \%$ & $5.6 \times 10^{-7}: 9 \%$ & i. $2 \times 10^{-5} \pm 7 \pi_{n}$ \\
\hline$s-318$ & $5.9 \times 10^{-9}+14 \%$ & $1.9 \times 10^{-10} \pm 48 \%$ & $2.4 \times 10^{-7} \div 12 \%$ & $2.4 \times 10^{-7} \pm 7 \%$ & $5.7 \times 10^{-7}=9 \%$ & $1.0 \times 10^{-5}: 7$ \\
\hline$s-319$ & $1.8 \times 10^{-7} \pm 7 \%$ & $5.5 \times 10^{-9}+9^{\sigma}$ & $2.8 \times 10^{-7} \div 12^{\circ}$ & $6.4 \times 10^{-7} \div 7^{\circ}$ & $7.2 \times 10^{-7}=10 \%$ & $1.2 \times 10^{-5}: \ldots$ \\
\hline$s-320$ & $1.4 \times 10^{-8}+7 \sigma_{m}^{\prime}$ & $9.5 \times 10^{-10}+17 \%$ & $0.1 \times 10^{-7}+5^{m}$ & $5.7 \times 10^{-7}=4$ & $5.7 \times 10^{-7}=7 \%$ & $1.4 \times 10^{-5}=4^{\pi}$ \\
\hline$S-223$ & $9.6 \times 10^{-9} \pm 12 \%$ & $8.5 \times 10^{-10}: 310_{0}$ & collected in 1972 a & same location as & 319 & \\
\hline $5-225$ & $6.8 \times 10^{-9}+11 \%$ & $5.4 \times 10^{-10}+28^{m}$ & collected in 1972 a & same location as & 318 & \\
\hline $\begin{array}{l}\text { Median } \\
\text { vâlue }\end{array}$ & $5.9 \times 10^{-9}$ & $4.8 \times 10^{-10}$ & $2.8 \times 10^{-7}$ & $5.8 \times 10^{-7}$ & $6.2 \times 10^{-7}$ & $1.1 \times 10^{-5}$ \\
\hline
\end{tabular}

${ }^{a}$ See Fig. 5 for sample locations. All samples were taken at a depth of $0-1 \mathrm{~cm}$. 
Table 6. Various radionuclides observed in San Joaquin Valley soils during 1973.

\begin{tabular}{|c|c|c|c|c|c|c|}
\hline \multirow[b]{2}{*}{ Location $^{a}$} & \multicolumn{3}{|c|}{ Deposition values, $\mu \mathrm{Ci} / \mathrm{Ni}^{2}$} & \multicolumn{2}{|c|}{ Uranium $\mu \mathrm{g} / \mathrm{g}$ dry soil } & \multirow[b]{2}{*}{$235 i / 238 v$} \\
\hline & ${ }^{239} \mathrm{Pu}$ & ${ }^{238} \mathrm{Pu}$ & ${ }^{137} \mathrm{Cs}$ & $235_{i}$ & ${ }^{238} \mathrm{~L}$ & \\
\hline$S-234$ & $8.9 \times 10^{-4} \pm 9 \%$ & $2.6 \times 10^{-5} \pm 39 \%$ & $1.7 \times 10^{-2}=5 \%$ & $1.4 \times 10^{-2} \pm 2^{\sigma}$ & $1.9 \neq 3 \%$ & $7.3 \times 10^{-3}$ \\
\hline$S-235$ & $2.0 \times 10^{-4} \pm 20 \mathrm{~m}$ & $5.1 \times 10^{-6} \pm 67 \%$ & $6.7 \times 10^{-3} \pm 10^{\sigma}$ & $1.3 \times 10^{-2} \pm 2 \pi$ & $1.9 \pm 3 \%$ & $7.3 \times 10^{-3}$ \\
\hline$S-236$ & $6.2 \times 10^{-4} \pm 11 \%$ & $2.6 \times 10^{-5}+41 \%$ & $2.8 \times 10^{-2}=15 \%$ & $1.3 \times 10^{-2} \pm 2 \%$ & $1.8 \pm 3 \%$ & $7.2 \times 10^{-3}$ \\
\hline$S-237$ & $6.0 \times 10^{-4} \pm 9 \%$ & $2.2 \times 10^{-5} \pm 34 \%$ & $2.7 \times 10^{-2} \pm 19 \%$ & $2.1 \times 10^{-2} \pm 2 \%$ & $2.9 \pm 3 \%$ & $7.2 \times 10^{-3}$ \\
\hline $5-238$ & $4.7 \times 10^{-4} \pm 15 \%$ & $1.8 \times 10^{-5} \pm 54 \%$ & $2.9 \times 10^{-2} \pm 22 \%$ & $6.4 \times 10^{-2} \pm 2 \%$ & $9.1: 3 \%$ & $7.2 \times 10^{-3}$ \\
\hline$S-239$ & $6.0 \times 10^{-4} \pm 12 \%$ & $1.7 \times 10^{-5}+52 \%$ & $3.0 \times 10^{-2}+18 \%$ & $1.7 \times 10^{-2} \pm 2 \%$ & $2.4 \pm 3 \%$ & $7.2 \times 10^{-3}$ \\
\hline$S-240$ & $5.9 \times 10^{-4}+10 \%$ & $2.6 \times 10^{-5} \pm 35 \%$ & $6.0 \times 10^{-2} \pm 17 \%$ & $7.2 \times 10^{-2} \pm 2 \%$ & $10.1 \pm 3 \%$ & $7.3 \times 10^{-3}$ \\
\hline$S-241$ & $1.1 \times 10^{-3} \pm 12 \%$ & $2.1 \times 10^{-5} \pm 50 \%$ & - & $1.8 \times 10^{-2} \pm 2 \%$ & $2.6 \pm 3 \%$ & $7.2 \times 10^{-3}$ \\
\hline S-242 & $1.6 \times 10^{-4} \pm 15 \%$ & $6.4 \times 10^{-6}+60 \%$ & $1.5 \times 10^{-3} \pm 27 \%$ & $1.2 \times 10^{-1} \pm 2 \%$ & $17.0 \pm 3 \%$ & $7.3 \times 10^{-3}$ \\
\hline$S-243$ & $9.9 \times 10^{-4} \pm 9 \%$ & $3.2 \times 10^{-5} \pm 20 \%$ & $1.0 \times 10^{-2} \pm 11 \%$ & $7.3 \times 10^{-2} \pm 270$ & $10.1 \pm 3 \%$ & $7.3 \times 10^{-3}$ \\
\hline$S-244$ & $6.5 \times 10^{-4} \pm 14 \%$ & $2.8 \times 10^{-5} \pm 58 \%$ & $2.9 \times 10^{-3} \pm 16_{\%}^{*}$ & $5.5 \times 10^{-2} \pm 2 \%$ & $7.6 \pm 3 \%$ & $7.3 \times 10^{-3}$ \\
\hline$S-245$ & $4.7 \times 10^{-4}+15^{0}$ & $1.4 \times 10^{-5} \pm 48 \%$ & $3.9 \times 10^{-3} \pm 21 \%$ & $6.3 \times 10^{-2} \pm 2 \%$ & $8.8 \pm 3 \%$ & $7.3 \times 10^{-3}$ \\
\hline$S-246$ & $8.5 \times 10^{-4} \pm 14 \%$ & $2.3 \times 10^{-5} \pm 58 \%$ & $3.2 \times 10^{-2}+15 \%$ & $4.2 \times 10^{-2} \pm 2 \%$ & $5.9 \pm 3 \%$ & $7.3 \times 10^{-3}$ \\
\hline$S-247$ & $9.6 \times 10^{-4} \pm 13 \%$ & $3.0 \times 10^{-5} \pm 49^{\circ}$ & $5.0 \times 10^{-2} \pm 10 \%$ & $5.5 \times 10^{-2} \pm 2 \%$ & $7.8 \pm 3 \%$ & $7.2 \times 10^{-3}$ \\
\hline$S-248$ & $1.2 \times 10^{-3} \pm 10 \%$ & $8.0 \times 10^{-5} \pm 32^{n}$ & $6.0 \times 10^{-2} \pm 9 \%$ & $4.0 \times 10^{-2} \pm 2 \%$ & $5.6 \pm 3 \%$ & $7.2 \times 10^{-3}$ \\
\hline$S-249$ & $1.1 \times 10^{-3} \pm 10 \%$ & $2.2 \times 10^{-5} \pm 37 \%$ & $5.3 \times 10^{-2} \pm 9 \%$ & $1.4 \times 10^{-2} \pm 2 \%$ & $2.0 \pm 3$ & $7.3 \times 10^{-3}$ \\
\hline$S-250$ & $2.4 \times 10^{-4} \pm 23 \%$ & $4.1 \times 10^{-5}+38 \%$ & $1.9 \times 10^{-3} \pm 41 \%$ & $4.1 \times: 0^{-2} \pm 2 \%$ & $5.7 \pm 3 \%$ & $7.2 \times 10^{-3}$ \\
\hline$S-251$ & $8.3 \times 10^{-4} \pm 11 \%$ & $5.6 \times 10^{-5} \pm 38^{\circ}$ & $4.0 \times 10^{-2} \pm 13 \%$ & $1.5 \times 10^{-2} \pm 2 \%$ & $2.1 \div 3 \%$ & $7.4 \times 10^{-3}$ \\
\hline $\begin{array}{l}\text { Median } \\
\text { value }\end{array}$ & $6.5 \times 10^{-4}$ & $2.6 \times 10^{-5}$ & $2.8 \times 10^{-2}$ & $4.0 \times 10^{-2}$ & 5.6 & $7.3 \times 10^{-3}$ \\
\hline
\end{tabular}

${ }^{\text {a }}$ See Fig. 6 for sample locations. dil samples were taten at a depth of $0-25 \mathrm{~cm}$.

${ }^{b}$ Ratios are atomic ratios; the isotopic atomic ratio of natural uranium is $7.25 \times 10^{-3}$. 
Table 7. Activity levels of various radionuclides in surface drainage ditches and crecks $(\mu \mathrm{Ci} / \mathrm{g})$.

\begin{tabular}{|c|c|c|c|c|}
\hline Location & $\begin{array}{c}\text { Depth } \\
(\mathrm{cm})\end{array}$ & ${ }^{239} \mathrm{Pu}$ & ${ }^{238} \mathrm{Pu}$ & ${ }^{137} \mathrm{Cs}$ \\
\hline$S-304$ & $0-1$ & $4.9 \times 10^{-9} \pm 7 \%$ & $2.6 \times 10^{-10} \pm 7 \%$ & $1.7 \times 10^{-8} \pm 56 \%$ \\
\hline$s-305$ & $s^{a}$ & $2.6 \times 10^{-9} \pm 8 \%$ & $1.6 \times 10^{-10} \pm 24 \sigma_{0}$ & $1.1 \times 10^{-7} \pm 24 \%$ \\
\hline$S-306$ & $s$ & $8.1 \times 10^{-10} \pm 20 \%$ & $3.5 \times 10^{-11} \pm 71 \%$ & $3.8 \times 10^{-8} \pm 32 \%$ \\
\hline $5-307$ & $\mathrm{~s}$ & $1.5 \times 10^{-9} \pm 14 \%$ & $6.5 \times 10^{-10} \pm 19 \%$ & $7.0 \times 10^{-6} \pm 2 \%$ \\
\hline$S-308$ & $s$ & $6.2 \times 10^{-10} \pm 22 \%$ & $3.8 \times 10^{-11} \pm 44 \%$ & $2.9 \times 10^{-8} \pm 60 \%$ \\
\hline$S-309$ & $s$ & $3.2 \times 10^{-9} \pm 15 \%$ & $5.0 \times 10^{-10} \pm 30 \%$ & $1.1 \times 10^{-6} \pm 7 \%$ \\
\hline$S-310$ & $0-1$ & $2.2 \times 10^{-8} \pm 5 \%$ & $1.5 \times 10^{-9} \pm 8 \%$ & $3.2 \times 10^{-8} \pm 36 \%$ \\
\hline$S-310$ & $0-25$ & $2.0 \times 10^{-8} \pm 7 \%$ & $1.3 \times 10^{-10} \pm 21 \%$ & $3.6 \times 10^{-9} \pm 100 \%$ \\
\hline $5-311$ & $0-1$ & $1.3 \times 10^{-8} \pm 5 \%$ & $8.8 \times 10^{-10} \pm 9 \%$ & $1.7 \times 10^{-8} \pm 70 \%$ \\
\hline$S-311$ & $0-25$ & $1.4 \times 10^{-9} \pm 8 \%$ & $8.8 \times 10^{-11} \pm 24 \%$ & - \\
\hline $5-312$ & $0-1$ & $6.8 \times 10^{-8} \pm 5 \%$ & $4.1 \times 10^{-9} \pm 8 \%$ & $5.7 \times 10^{-8}=10 \%$ \\
\hline$S-312$ & $0-25$ & $9.6 \times 10^{-9} \pm 7 \%$ & $7.7 \times 10^{-10} \pm 15 \%$ & $1.4 \times 10^{-8} \pm 100 \%$ \\
\hline$S-313$ & $0 \sim 1$ & $4.8 \times 10^{-8} \pm 10 \%$ & $6.5 \times 10^{-10} \pm 22 \%$ & $1.4 \times 10^{-7} \pm 17^{\circ}$ \\
\hline$S-313$ & $0-25$ & $1.3 \times 10^{-9} \pm 13 \%$ & $9.8 \times 10^{-11} \pm 41 \%$ & $5.7 \times 10^{-8} \pm 33 \%$ \\
\hline S-314 & $0-1$ & $1.1 \times 10^{-9} \pm 13 \%$ & $1.7 \times 10^{-10} \pm 29 \%$ & $4.4 \times 10^{-8} \pm 35 \%$ \\
\hline$S-315$ & $0-1$ & $4.9 \times 10^{-9} \pm 9 \%$ & $9.2 \times 10^{-10} \pm 16 \%$ & $9.9 \times 10^{-B} \pm 16 \%$ \\
\hline$S-315$ & $0-25$ & $7.6 \times 10^{-9} \pm 8 \%$ & $5.9 \times 10^{-10} \pm 20 \%$ & $7.5 \times 10^{-8} \pm 17 \%$ \\
\hline $5-316$ & $s$ & $1.8 \times 10^{-9} \pm 8 \%$ & $1.3 \times 10^{-10} \pm 25 \%$ & $5.3 \times 10^{-8} \pm 21 \%$ \\
\hline S-317 & $S$ & $2.7 \times 10^{-9} \pm 6 \%$ & $1.6 \times 10^{-10} \pm 20 \%$ & $5.2 \times 10^{-8} \pm 17 \%$ \\
\hline
\end{tabular}

${ }_{\mathrm{S}}^{\mathrm{a}}=$ Sediment sample representing a wet sample taken from the bottom of a running stream or creek. These samples were dried and activity is per gram of dry sample. All other samples were from dry ditch or creek beds. 
Table 8. Livermore sewage treatment plant sampling results during 1973.

\begin{tabular}{|c|c|c|c|c|c|c|c|c|}
\hline \multicolumn{9}{|c|}{ Gross alpha activity $(\mu \mathrm{C} i / \mathrm{ml})$} \\
\hline \multirow[b]{2}{*}{ Month } & \multirow{2}{*}{$\begin{array}{l}\text { No. } \\
\text { of } \\
\text { samples }\end{array}$} & \multicolumn{3}{|c|}{ Digesters } & \multirow{2}{*}{$\begin{array}{c}\text { No. } \\
\text { of } \\
\text { samples }\end{array}$} & \multicolumn{3}{|c|}{ Aeration tank } \\
\hline & & Maximum & Minimum & Average & & Maximum & Minimum & Average \\
\hline Jän. & 10 & $7.4 \times 10^{-7} \pm 34 \%$ & $1.7 \times 10^{-7} \pm 43 \%$ & $4.3 \times 10^{-7}$ & 5 & $1.1 \times 10^{-7} \pm 27 \%$ & $1.4 \times 10^{-8} \pm 30 \%$ & $7.7 \times 10^{-8}$ \\
\hline Feb. & 8 & $5.3 \times 10^{-7} \pm 30 \%$ & $4.7 \times 10^{-8} \pm 41 \%$ & $2.6 \times 10^{-7}$ & 4 & $7.9 \times 10^{-8} \pm 36 \%$ & $1.2 \times 10^{-8} \pm 30 \%$ & $5.1 \times 10^{-8}$ \\
\hline Mar. & 8 & $3.2 \times 10^{-7} \pm 32 \%$ & $1.6 \times 10^{-7} \pm 41 \%$ & $2.1 \times 10^{-7}$ & 4 & $1.2 \times 10^{-7} \pm 24 \%$ & $6.8 \times 10^{-8} \pm 34 \%$ & $9.7 \times 10^{-8}$ \\
\hline Apr. & 8 & $4.1 \times 10^{-7} \pm 29 \%$ & $1.2 \times 10^{-7} \pm 41 \%$ & $2.1 \times 10^{-7}$ & 4 & $7.7 \times 10^{-7} \pm 10 \%$ & $9.2 \times 10^{-8}+29 \%$ & $2.7 \times 10^{-8}$ \\
\hline May & 10 & $2.7 \times 10^{-7} \pm 28 \%$ & $1.2 \times 10^{-7} \pm 40 \%$ & $2.1 \times 10^{-7}$ & 5 & $9.0 \times 10^{-8} \pm 27 \%$ & $7.4 \times 10^{-8} \pm 34 \%$ & $8.4 \times 10^{-8}$ \\
\hline June & 8 & $5.3 \times 10^{-7} \pm 25 \%$ & $1.3 \times 10^{-7} \pm 34 \%$ & $2.9 \times 10^{-7}$ & 4 & $1.2 \times 10^{-7} \pm 24 \%$ & $7.8 \times 10^{-8} \pm 30 \%$ & $9.2 \times 10^{-8}$ \\
\hline July & 10 & $3.2 \times 10^{-7} \pm 31 \%$ & $2.6 \times 10^{-8} \pm 36 \%$ & $2.0 \times 10^{-7}$ & 5 & $1.7 \times 10^{-7} \pm 22 \%$ & $6.1 \times 10^{-8} \pm 32 \%$ & $9.3 \times 10^{-8}$ \\
\hline Aug. & 8 & $8.6 \times 10^{-7} \pm 28 \%$ & $2.3 \times 10^{-7} \pm 29 \%$ & $4.5 \times 10^{-7}$ & 4 & $1.1 \times 10^{-7} \pm 26 \%$ & $4.8 \times 10^{-8} \pm 38 \%$ & $6.9 \times 10^{-8}$ \\
\hline Sept. & 8 & $8.2 \times 10^{-7} \pm 17 \%$ & $2.1 \times 10^{-7} \pm 32 \%$ & $3.3 \times 10^{-7}$ & 4 & $5.9 \times 10^{-8} \pm 30 \%$ & $4.1 \times 10^{-8} \pm 43 \%$ & $4.7 \times 10^{-8}$ \\
\hline Oct. & 10 & $6.8 \times 10^{-7} \pm 27 \%$ & $1.8 \times 10^{-7} \pm 54 \%$ & $3.4 \times 10^{-7}$ & 5 & $6.0 \times 10^{-8} \pm 33 \%$ & $2.7 \times 10^{-8} \pm j 0 \%$ & $4.9 \times 10^{-8}$ \\
\hline Nov. & 8 & $3.0 \times 10^{-7} \pm 32 \%$ & $1.6 \times 10^{-7} \pm 37 \%$ & $2.1 \times 10^{-7}$ & 4 & $6.2 \times 10^{-8} \pm 36 \%$ & $3.4 \times 10^{-8} \pm 44 \%$ & $4.9 \times 10^{-8}$ \\
\hline Dec. & 8 & $4.5 \times 10^{-7} \pm 21 \%$ & $1.3 \times 10^{-7} \pm 39 \%$ & $2.5 \times 10^{-7}$ & 4 & $6.7 \times 10^{-8} \pm 32^{\%}$ & $2.5 \times 10^{-8} \pm 59 \%$ & $4.4 \times 10^{-8}$ \\
\hline
\end{tabular}


Table 9. Livermore sewage treatmeni plant sampling results during 1973.

\begin{tabular}{|c|c|c|c|c|c|c|c|c|c|c|c|c|c|}
\hline \multirow[b]{3}{*}{ Month } & \multicolumn{13}{|c|}{ Gross beta activity $(\mu \mathrm{Ci} / \mathrm{ml})$} \\
\hline & \multirow{2}{*}{$\begin{array}{c}\text { No. of } \\
\text { Samples }\end{array}$} & \multicolumn{3}{|c|}{ Digester } & \multirow{2}{*}{$\begin{array}{c}\text { No. of } \\
\text { Sampes }\end{array}$} & \multicolumn{3}{|c|}{ Aeration tank } & \multirow{2}{*}{$\begin{array}{c}\text { No. of } \\
\text { Samples }\end{array}$} & \multicolumn{3}{|c|}{ Erfluent } & \multirow{2}{*}{$\mathrm{Reg}^{i 0}$} \\
\hline & & Maximum & Misimum & Average S & & Maximum & Minimum & Average: & & Maximum & Minimum & Average & \\
\hline Jan. & 10 & $4.6 \times 10^{-7} \pm 15 \%$ & $2.1 \times 10^{-7} \pm 15 \%$ & $3.3 \times 10^{-7}$ & 5 & $5.5 \times 10^{-8} \pm 7 \%$ & $4.2 \times 10^{-8} \pm 8 \%$ & $4.8 \cdot 10^{-8}$ & 12 & $2.1 \times 10^{8}+32 \%$ & $7.2 \times 10^{-9} \pm 100 \%$ & $1.3 \times 10^{-8}$ & 13 \\
\hline Feb. & 8 & $2.3 \times 10^{-7} \pm 6 \%$ & $1.5 \times 10^{-7}+16 \%$ & $2.1 \times 10^{-7}$ & 4 & $5.0 \times 10^{-8} \pm 7 \%$ & $4.4 \times 10^{-8} \pm 85$ & $4.7 \times 10^{-8}$ & 8 & $2.5 \times 10^{-8} \pm 30 \%$ & $9.8 \times 10^{-9} \pm 64^{\pi}$ & $1.8 \times 10^{-8}$ & 18 \\
\hline Mar. & 8 & $5.4 \times 10^{-7} \pm 9 \%$ & $1.8 \times 10^{-7} \pm 14 \%$ & $3.1 \times 10^{-7}$ & 4 & $5.5 \times 10^{-8} \pm 7 \%$ & $4.2 \times 10^{-8} \pm 87$ & $5.0 \times 10^{-8}$ & 12 & $2.5 \times 10^{-8} \pm 30 \%$ & $7.0 \times 10^{-9} \pm 100 \%$ & $1.4 \times 10^{-8}$ & 14 \\
\hline Apr. & 8 & $1.3 \times 10^{-5} \pm 6 \%$ & $1.5 \times 10^{-7} \pm 17 \%$ & $3.6 \times 10^{-7}$ & 4 & $5.8 \times 10^{-8} \pm 7 \%$ & $4.8 \times 10^{-8} \pm 8 \%$ & $5.3 \times 10^{-8}$ & 10 & $2.5 \times 10^{-8} \div 28 F_{0}$ & $8.1 \times 10^{-9}=100 \%$ & $1.7 \times 10^{-8}$ & 17 \\
\hline May & 10 & $4.3 \times 10^{-7} \pm 13 \%$ & $1.7 \times 10^{-7} \pm 14 \%$ & $2.4 \times 10^{-7}$ & 5 & $5.3 \times 10^{-8} \pm 8 \%$ & $3.8 \times 10^{-8}+9 \%$ & $4.5 \times 10^{-8}$ & 8 & $3.1 \times 10^{-8} \pm 28 \%$ & $1.1 \times 10^{-8} \pm 59 \%$ & $1.9 \times 10^{-8}$ & 19 \\
\hline June & 8 & $4.1 \times 10^{-7} \pm 12 \%$ & $1.8 \times 10^{-7} \pm 14 \%$ & $3.0 \times 10^{-7}$ & 4 & $5.3 \times 10^{-8} \pm 8 \%$ & $4.4 \times 10^{-8} \pm 9 \%$ & $4.9 \times 10^{-8}$ & 13 & $3.1 \times 10^{-8} \times 27 \%$ & $7.3 \times 10^{-9}+1007$ & $1.7 \times 10^{-8}$ & 17 \\
\hline July & 10 & $4.2 \times 10^{-7} \pm 13 \%$ & $2.1 \times 10^{-7} \pm 12 \%$ & $3.1 \times 10^{-7}$ & 5 & $1.2 \times 10^{-1} \pm 5 \%$ & $4.6 \times 10^{-8} \pm 8 \%$ & $6.7 \times 10^{-7}$ & 9 & $2.8 \times 10^{-8} \pm 30 \%$ & $1.4 \times 10^{-8} \pm 47 \%$ & $1.7 \times 10^{-8}$ & 17 \\
\hline Aug. & B & $1.2 \times 10^{-6} \pm 12 \%$ & $3.7 \times 10^{-7} \pm 10 \%$ & $6.1 \times 10^{-7}$ & 4 & $1.1 \times 10^{-7} \pm 5 \%$ & $7.1 \times 10^{-8} \pm 7 \%$ & $8.6 \times 10^{-8}$ & 12 & $6.8 \times 10^{-8} \pm 17 \%$ & $8.3 \times 10^{-9} \pm 100 \%$ & $2.5 \times 10^{-8}$ & 25 \\
\hline Sept. & 8 & $6.1 \times 10^{-7} \pm 10 \%$ & $2.9 \times 10^{-7} \pm 1370$ & $3.8 \times 10^{-7}$ & 4 & $5.3 \times 10^{-8} \pm 8 \%$ & $3.9 \times 10^{-8} \pm 9 \%$ & $4.7 \times 10^{-8}$ & 12 & $3.6 \propto 10^{-8} \pm 26 \%$ & $7.9 \times 10^{-9} \pm 100 \%$ & $1.9 \times 10^{-8}$ & 19 \\
\hline Oct. & 10 & $5.1 \times 10^{-7} \pm 14 \%$ & $2.4 \times 10^{-7} \pm 14 \%$ & $3.5 \times 10^{-7}$ & 5 & $4.9 \times 10^{-8}+7 \%$ & $3.1 \times 10^{-8} \pm 97$ & $4.1 \times 10^{-8}$ & 11 & $1.8 \times 10^{-8} \pm 39 \%$ & $8.1 \times 10^{-9} \pm 100 \%$ & $1.2 \times 10^{-8}$ & 12 \\
\hline Nov. & 8 & $3.6 \times 10^{-7} \pm 16 \%$ & $1.9 \times 10^{-7} \pm 18 \%$ & $2.5 \times 10^{-7}$ & 4 & $3.9 \times 10^{-8} \pm 97$ & $3.3 \times 10^{-8}+10 \%$ & $3.5 \times 10^{-8}$ & 8 & $1.8 \times 10^{-8} \pm 40 \%$ & $7.8 \times 10^{-9} \pm 100 \%$ & $1.2 \times 10^{-8}$ & 12 \\
\hline Dec. & 8 & $3.4 \times 10^{-7} \pm 18 \%$ & $1.5 \times 10^{-7} \pm 24 \%$ & $2.1 \times 10^{-7}$ & 4 & $3.4 \times 10^{-8} \pm 97$ & $2.8 \times 10^{-8} \pm 11 \%$ & $3.0 \times 10^{-8}$ & 12 & $2.1 \times 10^{-8}=350$ & $1.1 \times 10^{-8} \pm 54 \%$ & $1.4 \times 10^{-8}$ & 14 \\
\hline
\end{tabular}


Table 10. Transuranium elements observed in the Livermore Sewage Treatment Plant during $1973(\mu \mathrm{Ci} / \mathrm{ml})$.

\begin{tabular}{|c|c|c|c|c|c|}
\hline Month & Sinmple & ${ }^{230} \mathrm{Pu}$ & $23 \mathrm{fr}_{\mathrm{m}}$ & $2+1 \mathrm{Am}$ & ${ }^{2+4} \mathrm{~cm}$ \\
\hline \multirow[t]{4}{*}{ tan. } & Difester "1 & $0.9 \times 10^{-n}: 7 \pi$ & $3.2 \times 10^{-0}: 7^{n}$ & & \\
\hline & Difester $" 2$ & $9.6 \times 10^{-9} \pm 8^{\pi_{n}}$ & $1.1 \times 10^{-8}=8 \pi$ & $1.1 \times 10^{-1}=18^{\circ}$ & $2.7 \times 10^{-10}+22^{20}$ \\
\hline & Aerntor & $1.3 \times 10^{-10}=7^{n}$ & $5.8 \times 10^{-10}+8^{m}$ & $1,7 \times 10^{-10}=2.40$ & \\
\hline & Fffluent & $1 \times 10^{-11}: 21^{0}$ & $3 .+\times 10^{-12}+32^{\alpha}$ & $2.2 \times 10^{-12}=27 \%$ & $5.6 \times 10^{-12}=18^{5}$ \\
\hline \multirow[t]{4}{*}{ Fob. } & Digestur 1 & $6.3 \times 10^{-9} \div 6^{n^{-}}$ & $2.2 \times 10^{-n}+7^{\infty}$ & $7.5 \times 10^{-10}+210$ & $1.8 \times 10^{-n}: 19^{r}$ \\
\hline & Migestert 2 & $5.5 \times 10^{-n} \div 7^{\infty}$ & $1.7 \times 10^{-9}=70^{\sigma}$ & $1.8 \times 10^{-8}: 11^{-}$ & $2.0 \times 10^{-n}=10^{\sigma}$ \\
\hline & Aerator & $1.1 \times 10^{-3}=7^{\circ}$ & $6.9 \times 10^{-10}=80$ & $1.3 \times 10^{-10}=37 \%$ & $1.8 \times 10^{-10}=3.3 \%$ \\
\hline & Foluent & $1.3 \times 10^{-11} \div 10^{n}$ & $6 . \therefore \times 10^{-12} \pm 13^{\circ}$ & & \\
\hline \multirow[t]{4}{*}{ Mar. } & bigester 1 & $8.3 \times 10^{-n} \pm 6^{r}$ & $3.6 \times 10^{-9}=7^{m}$ & & \\
\hline & bigester 2 & $6.4 \times 10^{-9}=60$ & $4.2 \times 10^{-9}+7^{m}$ & $7.6 \times 10^{-10}=9^{\alpha}$ & $7.2 \times 10^{-10} ; 9^{9}$ \\
\hline & Aerator & $2.3 \times 10^{-3} \pm 7 \sigma_{n}$ & $1.3 \times 10^{-19}: 7 \%$ & & \\
\hline & Errluent & $1.8 \times 10^{-11}+8^{n^{*}}$ & $1,2 \times 10^{-11}=8$. & & \\
\hline \multirow[t]{3}{*}{ Apr. } & Bigrester" 1 & $7.8 \times 10^{-11}=70$ & $3.6 \times 10^{-9}=8 \pi$ & & \\
\hline & Digestir "2 & $6.4 \times 10^{-9}=B^{a}$ & $3.2 \times 10^{-9}=0^{\sigma}$ & & \\
\hline & Aerator & $1.6 \times 10^{-9} \pm 7^{\circ}$ & $B .5 \times 10^{-10}=0$ & & \\
\hline \multirow{4}{*}{ May } & $\begin{array}{l}\text { Fimueni } \\
\text { Direster } 49\end{array}$ & $2.8 \times 10^{-1}=80$ & $1.2 \times 10^{-11}=10^{-1}$ & & \\
\hline & Digester 42 & $5.6 \times 10^{-9} \pm 6^{\sigma}$ & $2.5 \times 10=10^{-9} \div 6 \%$ & & \\
\hline & Ac ator & $9.1 \times 10^{-10}=7 \%$ & $4.3 \times 10^{-10}=8^{0}$ & & \\
\hline & Efnuent & $7 .=\times 10^{-12} \pm 13 \sigma_{r}$ & $3,0 \times 10^{-12} \pm 19^{\circ}$ & & \\
\hline \multirow[t]{4}{*}{ Iune } & Digester "I & $1.2 \times 10^{-6}+4 \pi$ & $2.3 \times 10^{-9} \pm 5^{n}$ & & \\
\hline & Digester" 2 & $7.2 \times 10^{-n} \pm 4 \pi$ & $2.5 \times 10^{-9}=5^{n}$ & & \\
\hline & Aerator & $1.8 \times 10^{-13}=5 \%$ & $5.4 \times 10^{-10} \pm 6^{n}$ & & \\
\hline & E:fluent & $1.2 \times 10^{-11} \pm 13^{0}$ & $7.2 \times 10^{-12}+16^{\circ}$ & & \\
\hline \multirow[t]{4}{*}{ July } & Digester "l & $7.4 \times 10^{-3}+5 \sigma_{r}$ & $1.9 \times 10^{-9}=5$ & & \\
\hline & Difester 42 & $6.0 \times 10^{-3}+7 n$ & $1.9 \times 10^{-9}=70^{*}$ & & \\
\hline & Aerator & $1.1 \times 10^{-8}=70$ & $3.9 \times 10^{-10}, 9^{\sigma}$ & & \\
\hline & Effluent & $7.5 \times 10^{-12} \pm 15^{11}$ & $3 . B \times 10^{-12} \pm 15^{\circ}$ & & \\
\hline \multirow[t]{4}{*}{ Mug. } & Digester $\left.{ }^{b}\right]$ & $8.9 \times 10^{-n} \pm 9 \sigma$ & $2.2 \times 10^{-9} \div 10^{\sigma}$ & $1.4 \times 10^{-9}: 8^{3 \prime \prime}$ & $2.5 \times 10^{-10}+6^{5}$ \\
\hline & Digester id 2 & $9.5 \times 10^{-n} \div 5 "$ & $1 . n \times 10^{-9}=5^{n}$ & $2.2 \times 10^{-8} \cdot 7^{\sigma}$ & $2,3 \times 10^{-10}+9^{\circ}$ \\
\hline & Aerator & $5.4 \times 10^{-10}=6^{a}$ & $2.1 \times 10^{19}=70$ & $1.0 \times 10^{-10}: 10^{\circ}$ & $8,3 \times 10^{-12}=36 \sigma_{\mathrm{a}}$ \\
\hline & Errluent & $4.4 \times 10^{-12}=15^{\prime \prime} 0$ & $1.1 \times 10^{-12} \pm 27 \%$ & $0.7 \times 10^{-13}: 44^{0}$ & $1.2 \times 10^{-13}+90^{0}$ \\
\hline \multirow[t]{4}{*}{ Sept. } & Digester $d$ & $3.6 \times 10^{-1} \pm 50$ & $8.7 \times 10^{-10} \pm 5 \%$ & & \\
\hline & Digester $* 2$ & Lost & Lost & & \\
\hline & Aerator & $9.8 \times 10^{-11} \pm 80^{\prime \prime}$ & $8.7 \times 10^{-11} \pm 13 \%$ & & \\
\hline & Effluent & $4.8 \times 10^{-11}=66^{2}$ & $4.0 \times 10^{-12} \pm 8$ & & \\
\hline \multirow{3}{*}{ Oct. } & Digester 1 & $5.4 \times 10^{-9}+5 \%$ & $8.9 \times 10^{-10} \pm 50$ & & \\
\hline & Digester $>2$ & $6.3 \times 10^{-9} \pm 5^{0,}$ & $1.1 \times 10^{-9} \pm 5 \%$ & & \\
\hline & Aerator & $4.2 \times 10^{-10} \pm 0^{6}$ & $1.1 \times 10=8 \%$ & & \\
\hline \multirow[t]{4}{*}{ Nov. } & Digester & $\begin{array}{l}9.5 \times 10 \pm 15 \\
+2 \times 10^{-9}+70\end{array}$ & $\begin{array}{l}1.8 \times 10 \\
6.3 \times 10^{-10} \pm 29 \%\end{array}$ & & \\
\hline & Digester $=2$ & $3.2 \times 10^{-9} \pm 6 \%$ & $5.7 \times 10^{-10} \pm 7 \%$ & & \\
\hline & Aerator & $3.2 \times 10^{-10} \pm 9 \%$ & $4.6 \times 10^{-11} \pm 19 \%$ & & \\
\hline & Effluent & $2.1 \times 10^{-12} \pm 33^{\circ}$ & $2.7 \times 30^{-13} \pm 58 \%$ & & \\
\hline \multirow[t]{4}{*}{ Dec. } & Oigester ${ }^{H} 1$ & $6.13 \times 10^{-4} \pm 7 \%$ & $6.7 \times 10^{-10} \pm 9 \%$ & & \\
\hline & Digester $* 2$ & $3.7 \times 10^{-9} \pm 8^{5}$ & $4.8 \times 10^{-10}+10^{0}$ & & \\
\hline & Aerator & $3.3 \times 30^{-10}=12^{5}$ & $5.6 \times 10^{-11} \pm 23^{\circ}$ & & \\
\hline & Effluent & $3.2 \times 10^{-12} \pm 28^{\circ}$ & $1.2 \times 10^{-12} \pm 43^{\sigma}$ & & \\
\hline
\end{tabular}


Table 11. Comparison of various radionuclides in LLL and Livermore treatment plant effluents during $1973(\mu \mathrm{Ci} / \mathrm{m} 1)$.

\begin{tabular}{|c|c|c|c|c|c|c|}
\hline \multirow[b]{2}{*}{ Month } & \multicolumn{2}{|c|}{${ }^{239} \mathrm{Pu}$} & \multicolumn{2}{|c|}{${ }^{90} \mathrm{Sr}$} & \multicolumn{2}{|c|}{ Tritium } \\
\hline & LLL & Treatment plant & LLL & Treatment plant & LLL & Treatment plant \\
\hline $\mathrm{J}$ an. & $5.1 \times 10^{-10}$ & $1.1 \times 10^{-11}$ & $1.9 \times 10^{-9}$ & $3.0 \times 10^{-10}$ & $8.5 \times 10^{-5}$ & $3.6 \times 10^{-6}$ \\
\hline Feb. & $8.7 \times 10^{-10}$ & $1.3 \times 10^{-11}$ & $7.0 \times 10^{-10}$ & $7.0 \times 10^{-10}$ & $1.1 \times 10^{-5}$ & $2.8 \times 10^{-6}$ \\
\hline Mar. & $9.0 \times 10^{-10}$ & $1.8 \times 10^{-11}$ & $1.8 \times 10^{-9}$ & $6.6 \times 10^{-9}$ & $1.3 \times 10^{-5}$ & $3.3 \times 10^{-6}$ \\
\hline Apr. & $7.2 \times 10^{-10}$ & $2.8 \times 10^{-11}$ & $1.5 \times 10^{-9}$ & $7.2 \times 10^{-10}$ & $1.1 \times 10^{-4}$ & $7.0 \times 10^{-6}$ \\
\hline May & $3.6 \times 10^{-10}$ & $7.5 \times 10^{-12}$ & Lost & $6.0 \times 10^{-10}$ & $1.2 \times 10^{-5}$ & $1.3 \times 10^{-6}$ \\
\hline June & $5.9 \times 10^{-10}$ & $1.2 \times 10^{-11}$ & $3.6 \times 10^{-9}$ & $4.0 \times 10^{-10}$ & $4.0 \times 10^{-5}$ & $4.6 \times 10^{-6}$ \\
\hline July & $2.7 \times 10^{-10}$ & $7.5 \times 10^{-12}$ & $1.9 \times 10^{-9}$ & $7.0 \times 10^{-10}$ & $2.3 \times 10^{-5}$ & $1.8 \times 10^{-6}$ \\
\hline Aug. & $4.1 \times 10^{-10}$ & $4.4 \times 10^{-12}$ & $1.3 \times 10^{-12}$ & $9.0 \times 10^{-10}$ & $2.7 \times 10^{-5}$ & $3.3 \times 10^{-6}$ \\
\hline Sept. & $1.8 \times 10^{-10}$ & $4.8 \times 10^{-11}$ & $6.4 \times 10^{-9}$ & $5.0 \times 10^{-11}$ & $3.5 \times 10^{-5}$ & $3.3 \times 10^{-6}$ \\
\hline Oct. & $2.0 \times 10^{-11}$ & $9.5 \times 10^{-12}$ & $1.2 \times 10^{-9}$ & $5.0 \times 10^{-10}$ & $2.0 \times 10^{-4}$ & $3.2 \times 10^{-6}$ \\
\hline Nov. & $1.6 \times 10^{-10}$ & $2.1 \times 10^{-12}$ & $1.3 \times 10^{-9}$ & $2.0 \times 10^{-10}$ & $1.1 \times 10^{-5}$ & $1.8 \times 10^{-6}$ \\
\hline Dec. & $1.4 \times 10^{-11}$ & $3.2 \times 10^{-12}$ & $7.0 \times 10^{-9}$ & $1.1 \times 10^{-9}$ & $1.8 \times 10^{-5}$ & $3.8 \times 10^{-6}$ \\
\hline Annual Av & $4.1 \times 10^{-10}$ & $1.3 \times 10^{-11}$ & $2.1 \times 10^{-9}$ & $1.1 \times 10^{-9}$ & $4.9 \times 10^{-5}$ & $3.3 \times 10^{-6}$ \\
\hline RCG & $3 \times 10^{-5}$ & $3 \times 10^{-5}$ & $3 \times 10^{-7}$ & $3 \times 10^{-7}$ & $3 \times 10^{-3}$ & $3 \times 10^{-3}$ \\
\hline$\%$ RCG & $1.4 \times 10^{-3}$ & $4.3 \times 10^{-4}$ & $7.0 \times 10^{-1}$ & $3.7 \times 10^{-1}$ & 1.6 & $1.1 \times 10^{-1}$ \\
\hline
\end{tabular}


Table 12. Gross beta activities in Livermore water samples $(\mu \mathrm{Cl} / \mathrm{ml})$.

\begin{tabular}{|c|c|c|c|c|c|c|c|c|c|c|}
\hline \multirow{2}{*}{$\begin{array}{l}\text { Loca- } \\
\text { tion }\end{array}$} & \multirow{2}{*}{$\begin{array}{c}\text { No. } \\
\text { of } \\
\text { samples }\end{array}$} & \multicolumn{3}{|c|}{ January-June } & \multirow{2}{*}{$\begin{array}{c}\text { No. } \\
\text { of } \\
\text { samples }\end{array}$} & \multicolumn{3}{|c|}{ July-December } & \multirow{2}{*}{$\begin{array}{l}\text { Annual } \\
\text { average }\end{array}$} & \multirow{2}{*}{$\mathrm{RCG}^{\mathrm{F}}$} \\
\hline & & Maximum & Minimum & Average & & Maximum & Minimum & Average & & \\
\hline 11 & 6 & $2.1 \times 10^{-9}+34 \%$ & $1.6 \times 10^{-9} \pm 100 \%$ & $2.5 \times 10^{-9}$ & 6 & $3.4 \times 10^{-9} \pm 41 \%$ & $1.8 \times 10^{-7} \pm 100^{\%}$ & $2.7 \times 10^{-9}$ & $2.6 \times 10^{-9}$ & 8 \\
\hline 13 & 6 & $6.0 \times 10^{-9} \pm 28 \%$ & $1.5 \times 10^{-9} \pm 100 \%$ & $3.0 \times 10^{-9}$ & 6 & $2.7 \times 10^{-9} \pm 49 \%$ & $1.5 \times 10^{-9} \pm 100 \%$ & $1.8 \times 10^{-9}$ & $2,4 \times 10^{-9}$ & 8 \\
\hline 15 & 6 & $5.9 \times 10^{-9} \pm 28 \%$ & $2.3 \times 10^{-6} \pm 54 \%$ & $4.2 \times 10^{-9}$ & 6 & $3.1 \times 10^{-9} \pm 47 \%$ & $1.7 \times 10^{-9}+74 \%$ & $2.5 \times 10^{-9}$ & $3.3 \times 10^{-9}$ & 11 \\
\hline 16 & 6 & $6.6 \times 10^{-9} \pm 24 \%$ & $3.0 \times 10^{-8}+47 \%$ & $4.5 \times 10^{-9}$ & 6 & $4.0 \times 10^{-9} \pm 38 \%$ & $1.8 \times 10^{-9} \pm 100 \%$ & $2.9 \times 10^{-9}$ & $3.7 \times 10^{-9}$ & 12 \\
\hline 17 & 6 & $4.3 \times 10^{-9} \pm 34 \%$ & $1.6 \times 10^{-9} \pm 69 \%$ & $2.8 \times 10^{-9}$ & 6 & $6.9 \times 10^{-9} \pm 27 \%$ & $2.0 \times 10^{-9} \pm 68 \%$ & $4.0 \times 10^{-9}$ & $3.4 \times 10^{-9}$ & 11 \\
\hline 18 & 3 & $5.0 \times 10^{-9} \pm 29 \%$ & $3.9 \times 10^{-9} \pm 35^{m}$ & $4.6 \times 10^{-9}$ & $-{ }^{b}$ & & & & & \\
\hline 19 & 3 & $4.3 \times 10^{-9} \pm 32 \%$ & $1.6 \times 10^{-9} \pm 100 \%$ & $2.5 \times 10^{-9}$ & 6 & $4.8 \times 10^{-9}+35 \%$ & $1.4 \times 10^{-9} \pm 75 \%$ & $2.4 \times 10^{-9}$ & $2.5 \times 10^{-9}$ & 8 \\
\hline 20 & 4 & $6.8 \times 10^{-9} \pm 25 \%$ & $1.6 \times 10^{-9} \pm 64 \%$ & $4.5 \times 10^{-9}$ & 3 & $5.7 \times 10^{-9}+27 \%$ & $3.6 \times 10^{-9} \pm 39 \%$ & $4.7 \times 10^{-9}$ & $4.6 \times 10^{-9}$ & 15 \\
\hline 21 & 6 & $5.0 \times 10^{-9} \pm 29 \%$ & $2.4 \times 10^{-9} \pm 52 \%$ & $3.4 \times 10^{-9}$ & 6 & $5.0 \times 10^{-9}+32 \%$ & $1.9 \times 10^{-9} \pm 60 \%$ & $3.6 \times 10^{-9}$ & $3.5 \times 10^{-9}$ & 12 \\
\hline 22 & 3 & $1.0 \times 10^{-8} \pm 14 \%$ & $8.7 \times 10^{-9} \pm 20 \%$ & $9.6 \times 10^{-9}$ & $-{ }^{b}$ & & & & & \\
\hline 24 & 6 & $8.6 \times 10^{-9} \pm 20 \%$ & $1.6 \times 10^{-9} \pm 72 \%$ & $4.7 \times 10^{-9}$ & 6 & $7.0 \times 10^{-9} \pm 26 \%$ & $3.0 \times 10^{-9} \pm 49 \%$ & $2.5 \times 10^{-9}$ & $3.6 \times 10^{-9}$ & 12 \\
\hline 26 & 6 & $7.7 \times 10^{-9} \pm 20^{\%}$ & $1.6 \times 10^{-9} \pm 76 \%$ & $4.0 \times 10^{-9}$ & 6 & $3.8 \times 10^{-9} \pm 36 \%$ & $2.4 \times 10^{-9} \pm 57 \%$ & $3.0 \times 10^{-9}$ & $3.5 \times 10^{-9}$ & 12 \\
\hline
\end{tabular}

${ }^{\text {RCG (beta activity) }}=3.0 \times 10^{-B}$.

bo samples collected. 
Table 13. Tritium in water samples from Livermore Valley $1973(\mu \mathrm{Ci} / \mathrm{ml})$.

\begin{tabular}{|c|c|c|c|c|c|c|c|c|c|c|c|}
\hline \multirow[b]{2}{*}{ Loc ation } & \multirow{2}{*}{$\begin{array}{c}\text { No. of } \\
\text { samples }\end{array}$} & \multicolumn{3}{|c|}{ January-June } & \multirow{2}{*}{$\begin{array}{c}\text { No. of } \\
\text { samples }\end{array}$} & \multicolumn{3}{|c|}{ July-December } & \multirow{2}{*}{$\begin{array}{l}\text { tnnus' } \\
\text { averuge }\end{array}$} & \multirow[b]{2}{*}{$\% \mathrm{RCG}^{\mathrm{at}}$} & \multirow{2}{*}{$\begin{array}{l}\text { Calculated } \\
\text { innual } \\
\text { idult } \\
\text { vhole } \\
\text { body dose } \\
\text { (mrem) }\end{array}$} \\
\hline & & Maximum & Minimum & Average & & Maximum & Minimum & Average & & & \\
\hline 11 & 6 & $1.5 \times 10^{-7} \pm 5.6 \%$ & $1.1 \times 10^{-7} \pm 6.5 \%$ & $1.2 \times 10^{-7}$ & 5 & $1.8 \times 10^{-7} \pm 6.3 \%$ & $1.1 \times 10^{-7} \neq 8.7 \%$ & $1.4 \times 10^{-7}$ & $1.3 \times 10^{-7}$ & $4.3 \times 10^{-3}$ & $5.2 \times 10^{-3}$ \\
\hline 15 & 6 & $2.0 \times 10^{-7} \pm 5.2 \%$ & $1.6 \times 10^{-7} \pm 5.6 \%$ & $1.7 \times 10^{-7}$ & 5 & $1.6 \times 10^{-7} \pm 6.9 \%$ & $1.2 \times 10^{-7} \pm 6.8^{e}$ & $1.4 \times 10^{-7}$ & $1.6 \times 10^{-7}$ & $5.3 \times 10^{-3}$ & $6.4 \times 10^{-3}$ \\
\hline 16 & 6 & $2.8 \times 10^{-7} \pm 3.7 \%$ & $2.4 \times 10^{-7} \pm 4.4 \sigma_{i a}$ & $2.6 \times 10^{-7}$ & 5 & $2.4 \times 10^{-7} \pm 5.3 \%$ & $1.4 \times 10^{-7} \pm 7.5 \%$ & $1.9 \times 10^{-7}$ & $2.3 \times 10^{-7}$ & $7.7 \times 10^{-3}$ & $9.2 \times 10^{-3}$ \\
\hline 17 & 6 & $1.6 \times 10^{-7} \pm 10.2 \%$ & $1.1 \times 10^{-7} \pm 7.3 \%$ & $1.3 \times 10^{-7}$ & 5 & $1.2 \times 10^{-7} \pm 7.2 \%$ & $8.9 \times 10^{-8} \pm 9.9 \%$ & $1.0 \times 10^{-7}$ & $1.2 \times 10^{-7}$ & $4.0 \times 10^{-3}$ & $4.8 \times 10^{-3}$ \\
\hline 19 & 6 & $1.4 \times 10^{-7} \pm 6.0 \%$ & $5.8 \times 10^{-8} \pm 11.7 \%$ & $1.1 \times 10^{-7}$ & 5 & $2.1 \times 10^{-7} \pm 5.6 \%$ & $5.2 \times 10^{-8} \pm 17.5 \%$ & $1.1 \times 10^{-7}$ & $1.1 \times 10^{-7}$ & $3.7 \times 10^{-3}$ & $4.4 \times 10^{-3}$ \\
\hline 20 & 5 & $8.4 \times 10^{-7} \pm 2.7 \%$ & $3.1 \times 10^{-7}+8.8 \%$ & $6.2 \times 10^{-7}$ & 3 & $2.8 \times 10^{-7} \pm 14.5 \%$ & $1.1 \times 10^{-7} \pm 7.5 \%$ & $2.1 \times 10^{-7}$ & $4.6 \times 10^{-7}$ & $1.5 \times 10^{-2}$ & $1.8 \times 10^{-2}$ \\
\hline 21 & 6 & $1.6 \times 10^{-7} \pm 5.7 \%$ & $1.2 \times 10^{-7} \pm 7.0 \%$ & $1.4 \times 10^{-7}$ & $\mathbf{5}$ & $1.8 \times 10^{-7} \pm 6.5 \%$ & $1.3 \times 10^{-7} \pm 8.1 \%$ & $1.5 \times 10^{-7}$ & $1.4 \times 10^{-7}$ & $4.7 \times 10^{-3}$ & $5.6 \times 10^{-3}$ \\
\hline 22 & 3 & $2.5 \times 10^{-7} \pm 4.3 \%$ & $1.3 \times 10^{-7} \pm 6.8 \%$ & $1.7 \times 10^{-7}$ & 0 & & & & & & \\
\hline 24 & 6 & $1.1 \times 10^{-6} \pm 2.6 \%$ & $3.8 \times 10^{-7} \pm 5.3 \%$ & $6.2 \times 10^{-7}$ & 5 & $1.1 \times 10^{-6} \neq 3.0 \%$ & $2.5 \times 10^{-7} \pm 4.5 \%$ & $7.4 \times 10^{-7}$ & $6.7 \times 10^{-7}$ & $2.2 \times 10^{-2}$ & $2.7 \times 10^{-2}$ \\
\hline 26 & 6 & $2.0 \times 10^{-7} \pm 4.7 \%$ & $1.1 \times 10^{-7} \pm 7.4 \%$ & $1.5 \times 10^{-7}$ & 5 & $1.6 \times 10^{-7} \pm 7.1 \%$ & $1.2 \times 10^{-7} \pm 8.8 \%$ & $1.3 \times 10^{-7}$ & $1.4 \times 10^{-7}$ & $4.7 \times 10^{-3}$ & $5.6 \times 10^{-3}$ \\
\hline
\end{tabular}


Table 14. Tritium in vegetation samples from Livermore Valley $1973(\mu \mathrm{Ci} / \mathrm{g})$.

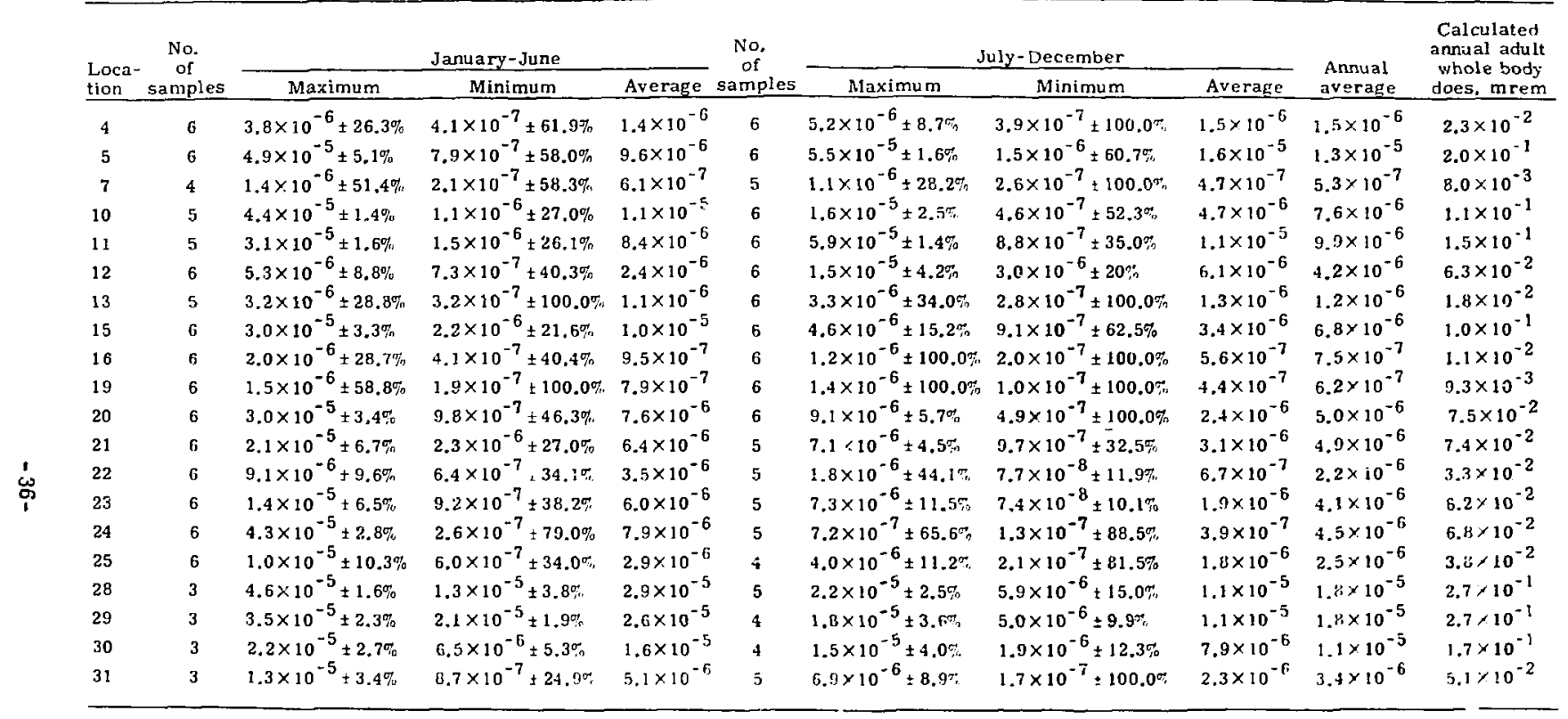


Table 15. Activities of various radionuclides in Livermore vegetation samples $(\mu \mathrm{Ci} / \mathrm{g}$ dry weight).

\begin{tabular}{|c|c|c|c|c|c|}
\hline Radionuclide & Maximum & Minimum & $\begin{array}{l}\text { Annual } \\
\text { average }\end{array}$ & $\begin{array}{l}\text { Calculated } \\
\text { annual } \\
\text { dose via } \\
\text { direct } \\
\text { togestion } \\
\text { (mrem) } \\
\end{array}$ & Critical organ \\
\hline${ }^{7} \mathrm{Be}$ & $8.3 \times 10^{-6}$ & $3.7 \times 10^{-7}$ & $2.1 \times 10^{-6}$ & 0.10 & Lower large intestine \\
\hline${ }^{40} \mathrm{~K}$ & $2 . E \times 10^{-5}$ & $8.2 \times 10^{-6}$ & $1.8 \times 10^{-5}$ & 25 & Whole body \\
\hline${ }^{95} \mathrm{Zr}$ & $1.7 \times 10^{-7}$ & $1.5 \times 10^{-8}$ & $3.5 \times 10^{-8}$ & 0.08 & Lower large intestine \\
\hline${ }^{103} \mathrm{Ru}$ & $1.8 \times 10^{-7}$ & $1.6 \times 10^{-8}$ & $8.4 \times 10^{-8}$ & 0.09 & Lower large intestine \\
\hline${ }^{137} \mathrm{Cs}$ & $8.8 \times 10^{-8}$ & $1.3 \times 10^{-8}$ & $4.7 \times 10^{-3}$ & 0.08 & Whole body \\
\hline${ }^{141} \mathrm{Ce}$ & $1.5 \times 10^{-7}$ & $1.9 \times 10^{-8}$ & $6.2 \times 10^{-8}$ & 0.06 & Lower large intestine \\
\hline${ }^{144} \mathrm{Ce}$ & $8.1 \times 10^{-7}$ & $3.0 \times 10^{-8}$ & $2.5 \times 10^{-8}$ & 0.20 & Lower large intestine \\
\hline
\end{tabular}

Table 16. Environmental radiation background exposure rate measurements at Lawrence Livermore Laboratory perimeters during 1973.

\begin{tabular}{|c|c|c|c|c|c|c|c|c|c|}
\hline \multirow[b]{2}{*}{$\begin{array}{l}\text { Loca- } \\
\text { tion }\end{array}$} & \multicolumn{2}{|c|}{ January -March } & \multicolumn{2}{|c|}{ April-June } & \multicolumn{2}{|c|}{ July-September } & \multicolumn{2}{|c|}{ October-December } & \multirow{2}{*}{$\begin{array}{l}\text { Annual } \\
\text { mrem/ } \\
\text { year }\end{array}$} \\
\hline & $\mathrm{mR} / \mathrm{hr}$ & $\begin{array}{l}\text { mrem/ } \\
\text { quarter }\end{array}$ & $\mathrm{mR} / \mathrm{hr}$ & $\begin{array}{l}\text { mrem/ } \\
\text { quarter }\end{array}$ & $\mathrm{mR} / \mathrm{hr}$ & $\begin{array}{l}\text { mrem/ } \\
\text { quarter }\end{array}$ & $\mathrm{mR} / \mathrm{hr}$ & $\begin{array}{c}\text { mrem/ } \\
\text { quarter }\end{array}$ & \\
\hline 1 & $1.2 \times 10^{-2}$ & 25 & $9.4 \times 10^{-3}$ & 19 & $6.8 \times 10^{-3}$ & 14 & $-{ }^{b}$ & - & $78^{c}$ \\
\hline 2 & $1.3 \times 10^{-2}$ & 27 & $1.0 \times 10^{-2}$ & 21 & $7.8 \times 10^{-3}$ & 16 & $9.2 \times 10^{-3}$ & 19 & 84 \\
\hline 3 & $1.1 \times 10^{-2}$ & 22 & $9.6 \times 10^{-3}$ & 20 & $7.8 \times 10^{-3}$ & 16 & $1.1 \times 10^{-2}$ & 22 & 81 \\
\hline 4 & $1.1 \times 10^{-2}$ & 22 & $8.3 \times 10^{-3}$ & 17 & $6.6 \times 10^{-3}$ & 13 & - & - & $70^{c}$ \\
\hline $5^{a}$ & $1.3 \times 10^{-2}$ & 27 & $1.2 \times 10^{-2}$ & 25 & $1.0 \times 10^{-2}$ & 21 & - & - & $96^{\mathrm{c}}$ \\
\hline 6 & $1.1 \times 10^{-2}$ & 22 & $7.6 \times 10^{-3}$ & 15 & $7.7 \times 10^{-3}$ & 16 & $8.8 \times 10^{-3}$ & 18 & 72 \\
\hline 7 & $7.7 \times 10^{-3}$ & 16 & $8.0 \times 10^{-3}$ & 16 & $-\mathrm{b}$ & - & $8.8 \times 10^{-3}$ & 18 & $69^{c}$ \\
\hline 8 & $8.8 \times 10^{-3}$ & 18 & $8.0 \times 10^{-3}$ & 16 & - & - & $8.4 \times 10^{-3}$ & 17 & $68^{c}$ \\
\hline 14 & $1.2 \times 10^{-2}$ & 25 & $1.1 \times 10^{-2}$ & 22 & $1.0 \times 10^{-2}$ & 21 & $1.0 \times 10^{-2}$ & 21 & 89 \\
\hline 15 & $1.2 \times 10^{-2}$ & 25 & $9.6 \times 10^{-3}$ & 20 & $9.0 \times 10^{-3}$ & 18 & $1.3 \times 10^{-2}$ & 27 & 90 \\
\hline Average & $1.1 \times 10^{-2}$ & 22 & $9.4 \times 10^{-3}$ & 19 & $8.2 \times 10^{-3}$ & 17 & $9.9 \times 10^{-3}$ & 20 & 80 \\
\hline
\end{tabular}

\footnotetext{
${ }^{\mathrm{a}}$ Neutron dose measurements (using an integrating rem meter) near Location 5 indicate an additional annual dese of approximately $250 \mathrm{mrem}$.

b - Indicates lost data.

c Projected data from 3 quarters.
} 
Table 17. Airborne particulate beta radioactivity at Site 300 during $1973(\mu \mathrm{Ci} / \mathrm{ml})$.

\begin{tabular}{|c|c|c|c|c|c|c|c|c|c|c|}
\hline \multirow{2}{*}{$\begin{array}{l}\text { Sampling } \\
\text { loc ittion }\end{array}$} & \multirow{2}{*}{$\begin{array}{c}\text { No. of } \\
\text { simples }\end{array}$} & \multicolumn{3}{|c|}{ January-Junc } & \multirow{2}{*}{$\begin{array}{c}\text { No. of } \\
\text { samples }\end{array}$} & \multicolumn{3}{|c|}{ Iuly-Dec mber } & \multirow{2}{*}{$\begin{array}{l}\text { Annuai } \\
\text { dverige }\end{array}$} & \multirow{2}{*}{$\mathrm{RCG}^{\mathrm{\pi}}$} \\
\hline & & Maximtum & Minimum & Average & & Maximum & Minimurs & Average & & \\
\hline 1 & 33 & $9.3 \times 10^{-14}+70$ & $7.6 \times 10^{-15} \pm 25^{n}$ & $2.3 \times 10^{-14}$ & 40 & $8.8 \times 10^{-14}=6 n^{2}$ & $1.1 \times 10^{-14} \div 15^{0}$ & $3.7 \times 10^{-14}$ & $3.0 \times 10^{-14}$ & 3 \\
\hline 2 & 31 & $4.7 \times 10^{-14} \pm 7 \sigma$ & $4.6 \times 10^{-15} \pm 15^{\circ}$ & $2.1 \times 10^{-14}$ & 40 & $7.2 \times 10^{-14} \pm 7 \%$ & $5.5 \times 10^{-15} \pm 695$ & $2.9 \times 10^{-14}$ & $2.5 \times 10^{-14}$ & 3 \\
\hline 3 & 34 & $4.9 \times 10^{-14}+11^{0.4}$ & $6.7 \times 10^{-15} \pm 14^{\sigma}$ & $1.9 \times 10^{-14}$ & 42 & $9.8 \times 10^{-14} \pm 8 \%$ & $4,1 \times 10^{-15} \pm 60^{\sigma}$ & $3.1 \times 10^{-14}$ & $2.5 \times 10^{-14}$ & 3 \\
\hline 4 & 32 & $5.0 \times 10^{-14}+10$ & $7.5 \times 10^{-15} \pm 18 \%$ & $2.3 \times 10^{-14}$ & 41 & $6.7 \times 10^{-14} \pm 6 \%$ & $5.3 \times 10^{-15} \div 64 \%$ & $3.2 \times 10^{-14}$ & $2.8 \times 10^{-14}$ & 3 \\
\hline 5 & 32 & $4.6 \times 10^{-14} \pm 9 \sigma_{i}$ & $6.9 \times 10^{-15}=29^{0}$ & $1.9 \times 10^{-14}$ & 40 & $8.1 \times 10^{-14} \pm 6_{i o}^{\sigma}$ & $6.1 \times 10^{-15} \pm 46 \%$ & $3.1 \times 10^{-14}$ & $2.5 \times 10^{-14}$ & 3 \\
\hline 6 & 22 & $1.3 \times 10^{-13}+7 \%$ & $6.8 \times 10^{-15} \pm 100 \%$ & $4.3 \times 10^{-14}$ & 20 & $6.8 \times 10^{-14} \pm 10^{\%}$ & $9.9 \times 10^{-15} \pm 17 \%$ & $9.1 \times 10^{-14}$ & $6.7 \times 10^{-14}$ & 7 \\
\hline 7 & 31 & $8.1 \times 10^{-14} \pm 13^{n t}$ & $5.7 \times 10^{-15}=30^{\circ}:$ & $2.4 \times 10^{-14}$ & 40 & $7.0 \times 10^{-14} \pm 7 \%$ & $5.8 \times 10^{-15} \pm 6,74$ & $3.4 \times 10^{-14}$ & $2.9 \times 10^{-14}$ & $:$ \\
\hline 8 & 33 & $1.0 \times 10^{-13} \pm 88^{0}$ & $1.7 \times 10^{-14} \pm 10^{\%}$ & $4.8 \times 10^{-14}$ & 42 & $2.4 \times 10^{-13} \pm 5 \%$ & $1.6 \times 10^{-14} \pm 19 \%$ & $7.2 \times 10^{-14}$ & $6.0 \times 10^{-14}$ & 6 \\
\hline 9 & 29 & $5.0 \times 10^{-14} \pm 8 \sigma_{\pi}$ & $3.2 \times 10^{-15} \pm 51 \%$ & $2.2 \times 10^{-14}$ & 42 & $7.1 \times 10^{-14} \pm 27 \%$ & $6.0 \times 10^{-15} \pm 100 \sigma^{\sigma}$ & $3.3 \times 10^{-14}$ & $2.8 \times 10^{-14}$ & 3 \\
\hline 10 & 35 & $4.4 \times 10^{-14} \pm 90^{\circ}$ & $7.4 \times 10^{-15} \pm 14 \%$ & $2.1 \times 10^{-14}$ & 42 & $6.5 \times 10^{-14} \pm 7 \%$ & $7.0 \times 10^{-15} \pm 12 \%$ & $2.9 \times 10^{-14}$ & $2.5 \times 10^{-14}$ & 3 \\
\hline 11 & 33 & $4.1 \times 10^{-i 4} \pm 9^{\sigma}$ & $2.4 \times 10^{-15} \pm 100^{\circ}$ & $2.0 \times 10^{-14}$ & 40 & $7.1 \times 10^{-14} \div 6 \%$ & $4.9 \times 10^{-15} \pm 100^{\circ} c$ & $3.3 \times 10^{-14}$ & $2.7 \times 10^{-14}$ & 3 \\
\hline Average & & & & $2.6 \times 10^{-14}$ & & & & $4.1 \times 10^{-14}$ & $3.4 \times 10^{-14}$ & 4 \\
\hline
\end{tabular}

"Spe Fig. 11 for locations.

${ }^{b} \mathrm{RCG}$ for airborne gross beta activity $=1.0 \times 10^{-12}$. 
Table 18. Results of gamma-ray spectral measurements of Site 300 air filters during $1973(\mu \mathrm{Ci} / \mathrm{ml})$.

\begin{tabular}{|c|c|c|c|c|c|c|c|c|c|}
\hline & ${ }^{144} \mathrm{Ce}$ & ${ }^{141} \mathrm{Ce}$ & $125 \mathrm{Sb}$ & $T_{\text {pe }}$ & ${ }^{103} \mathrm{Ru}$ & ${ }^{106} \mathrm{Ru}$ & ${ }^{137} \mathrm{Cs}$ & ${ }^{95} 7 . \mathrm{r}$ & $40 \mathrm{~K}$ \\
\hline Jan, & $1.7 \times 10^{-15} \pm 12^{\sigma}$ & $-^{\mathbf{a}}$ & $1.9 \times 10^{-16} \pm 14$ & $7.1 \times 10^{-14}+4$ & $6.9 \times 10^{-17} \div 50^{7}$ & $1.3 \times 10^{-15} \div 15^{m}$ & $5.6 \times 10^{-15}: 4 \%$ & $1.5 \times 10^{-16}: 19 \%$ & $3.4 \times 10^{-16}: 23^{r}$ \\
\hline Feb. & $2.4 \times 10^{-15} \pm 6 \%$ & - & $2.9 \times 10^{-16} \pm 10^{\%}$ & $0.1 \times 10^{-14} \pm 2 \%$ & $5.4 \times 10^{-17}=62 \%$ & $1.8 \times 10^{-15}: 9 \%$ & $1.4 \times 10^{-15}: 39$ & $2.0 \times 10^{-15}+44 \%$ & $3.7 \times 10^{-16} \cdot 26 \%$ \\
\hline Mar. & $1.1 \times 10^{-15} \pm 5 \%$ & - & $3.5 \times 10^{-15}+115$ & $8.9 \times 10^{-14} \div 2 \%$ & $3.2 \times 10^{-17}=50^{2}$ & $1.3 \times 10^{-15} \div 74$ & $1.0 \times 10^{-15} \cdot 8 \%$ & $9.3 \times 10^{-17} \cdot 190$ & $3.7 \times 10^{-16} \div 2 \mathrm{z}^{r}$ \\
\hline Apr. & $3.4 \times 10^{-15} \pm 8 \%$ & - & $5.1 \times 10^{-16} \pm B^{\circ}$ & $1.4 \times 10^{-13} \pm 2 \%$ & $3.6 \times 10^{-17}=38 \%$ & $2.6 \times 10^{-15}: 6^{-5}$ & $1.7 \times 10^{-15} \div 4 \%$ & $9.6 \times 10^{-17} \pm 48$ & $5.7 \times 10^{-16} \div 22^{7}$ \\
\hline May & $3.2 \times 10^{-15} \pm 5 \%$ & - & $4.7 \times 10^{-15} \pm 5$ & $1.4 \times 10^{-13} \pm 2 \%$ & - & $2.6 \times 10^{-15}=7$ & $1.6 \times 10^{-15}: 3 \%$ & $5.5 \times 10^{-17} \div 27 \%$ & $9.9 \times 10^{-16}=195$ \\
\hline Juле & $1.8 \times 10^{-15}+14^{\sigma}$ & - & $2.6 \times 10^{-16} \pm 25 \%$ & $1.0 \times 10^{-13} \pm 3 \%$ & - & $1.6 \times 10^{-15}=25^{-5}$ & $1.1 \times 10^{-15}=7^{2}$ & $3.8 \times 10^{-17}: 1007$ & $1.0 \times 10^{-15} \div 40-n$ \\
\hline July & $2.3 \times 10^{-15} \neq 21 \%$ & $1.6 \times 10^{-15} \div 22 \%$ & $4.2 \times 10^{-16} \div 24 \%$ & $1.5 \times 10^{-13}+1 . c_{1}$ & $3.0 \times 10^{-15}: 13 \%$ & $2.0 \times 10^{-15} \div 15 \%$ & $1.4 \times 10^{-15}=11 \%$ & $1.5 \times 10^{-15} \pm 28 \%$ & $1.0 \times 10^{-15}: 16 \%$ \\
\hline Aug. & $1.7 \times 10^{-15}+20 \%$ & $1.5 \times 10^{-15}=17 \%$ & $2.1 \times 10^{-16} \pm 30 \%$ & $1.1 \times 10^{-13} \pm 117$ & $2.6 \times 10^{-15}=11 \%$ & $1.4 \times 10^{-15}=25 \%$ & $8.1 \times 10^{-16}=\mathrm{cp}$ & $2.1 \times 10^{-15}: 6$ & $1.0 \times 10^{-15} \div 31$ \\
\hline Sept. & $9.1 \times 1 n^{-16} \pm 18 \%$ & $5.8 \times 10^{-16}+12 \%$ & $1.2 \times 10^{-16} \div 8 \%$ & $7.0 \times 10^{-14}+50$ & $1.2 \times 10^{-15}=10 \%$ & $8.1 \times 10^{-16}=33 \%$ & $+.3 \times 10^{-16}: 16^{\%}$ & $1.1 \times 10^{-15}+7 \%$ & $1.1 \times 10^{-15} \div 23^{5}$ \\
\hline Oct. & $1.7 \times 10^{-15}+22 \%$ & $1.7 \times 10^{-15}+15 \%$ & $9.9 \times 10^{-17}: 572$ & $1.5 \times 10^{-13} \neq 8$ & $2.9 \times 10^{-15} \pm 10^{7}$ & $1.3 \times 10^{-15} \pm 247$ & $4.4 \times 10^{-16} \div 18^{\pi}$ & $3.2 \times 10^{-15}, 6 \%$ & $1.0 \times 10^{-15} \neq 20 \pi$ \\
\hline Nov. & $1.2 \times 10^{-15} \pm 7 \%$ & $7.8 \times 10^{-16} \pm 7 \%$ & $6.9 \times 10^{-17} \div 38=$ & $6.1 \times 10^{-14}+2 \%$ & $1.4 \times 10^{-15}: 4 \%$ & $8.3 \times 10^{-16}=12 \%$ & $1.9 \times 10^{-16} \div 13 \%$ & $2.1 \times 10^{-15} \div 3 \%$ & $3.5 \times 10^{-16}+2 t \mathrm{pr} n$ \\
\hline$c c$ & $3.3 \times 10^{-15} \pm 10^{\circ}$ & $1.2 \times 10^{-15} \pm 11 \%$ & $1.6 \times 10^{-15}+18$ & $7.0 \times 10^{-14}=7 \%$ & $2.4 \times 10^{-15}=: 1 \%$ & $2.0 \times 10^{-15} \div 8$ & $3.3 \times 10^{-16} \div 8 \%$ & $+.5 \times 10^{-15}+2 \%$ & $3.0 \times 10^{-16}: 21 \%$ \\
\hline Anrupl av & $2.1 \times 10^{-15}$ & $=1.2 \times 10^{-15}$ & $2.6 \times 10^{-16}$ & $1.0 \times 10^{-13}$ & $1.4 \times 10^{-15}$ & $1.7 \times 10^{-15}$ & $9.2 \times 10^{-16}$ & $1.3 \times 10^{-15}$ & $7.1 \times 10^{-16}$ \\
\hline$\infty$ & $2 \times 7^{-10}$ & $5 \times 10^{-9}$ & $9 \times 10^{-10}$ & $4 \times 10^{-8}$ & $3 \times 10^{-9}$ & $2 \times 10^{-10}$ & $5 \times 10^{-10}$ & $1 \times 10^{-17}$ & $4 \times 10^{-9}$ \\
\hline$\% \pi C G$ & $1.0 \times 10^{-3}$ & $2.5 \times 10^{-5}$ & $2.8 \times 10^{-5}$ & $2.5 \times 10^{-4}$ & $4.7 \times 10^{-5}$ & $5.5 \times 10^{-4}$ & $1.8 \times 10^{-4}$ & $<1.3 \times 10^{-i}$ & $1.8 \times 10^{-5}$ \\
\hline
\end{tabular}

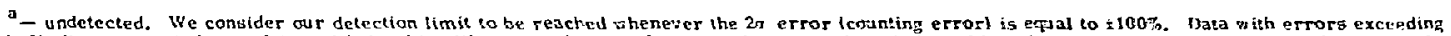

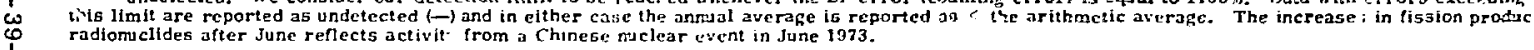


Table 19. Plutonium and uranium concentrations on air filters from Site 300 during 1973.

\begin{tabular}{|c|c|c|c|c|c|c|}
\hline \multirow[b]{2}{*}{ Month } & \multicolumn{2}{|c|}{ Activity $(\mu \mathrm{Ci} / \mathrm{mi})$} & \multirow[b]{2}{*}{${ }^{238} \mathrm{Pu},{ }^{239} \mathrm{Pu}$} & \multicolumn{2}{|c|}{ Mass (mg/mi } & \multirow[b]{2}{*}{$235 \mathrm{U}^{238} \mathrm{u}^{\mathrm{b}}$} \\
\hline & ${ }^{239} \mathrm{Pu}$ & ${ }^{238} \mathrm{Pu}$ & & ${ }^{235} \mathrm{U}$ & $238_{\mathrm{U}}$ & \\
\hline Jan. & $8.0 \times 10^{-18} \pm 7 \%$ & $1.0 \times 10^{-18} \pm 12 \%$ & $1.3 \times 10^{-1}$ & $1.4 \times 10^{-9} \pm 2 \%$ & $7.2 \times 10^{-7} \pm 5^{m}$ & $1.9 \times 10^{-3}$ \\
\hline Feb. & $1.8 \times 10^{-17} \pm 6 \%$ & $1.7 \times 10^{-13}+110^{\circ}$ & $9.4 \times 10^{-2}$ & $4.6 \times 10^{-10} \pm 2 \%$ & $1.6 \times 10^{-7} \pm 5^{\sigma}$ & $2.9 \times 10^{-3}$ \\
\hline Mar. & $1.5 \times 10^{-17} \pm 6 \%$ & $1.8 \times 10^{-18} \pm 11 \%$ & $1.2 \times 10^{-1}$ & $1.5 \times 10^{-10} \pm 3 \%$ & $3.6 \times 10^{-8} \pm 3^{0}$ & $4.2 \times 10^{-3}$ \\
\hline Apr. & $2.5 \times 10^{-17} \pm 7 \%$ & $2.6 \times 10^{-18} \pm 10_{\%}^{\%}$ & $1.0 \times 10^{-1}$ & $4.4 \times 10^{-10} \pm 2^{\circ}$ & $1.0 \times 10^{-7} \pm 4 \%$ & $4.4 \times 10^{-3}$ \\
\hline May & $2.3 \times 10^{-17} \pm 7 \%$ & $2.9 \times 10^{-18} \pm 10 \%$ & $1.3 \times 10^{-1}$ & $1.5 \times 10^{-9} \pm 2 \sigma_{i j}^{i}$ & $4.3 \times 10^{-7} \pm 4_{n}^{\sigma_{n}}$ & $3.5 \times 10^{-3}$ \\
\hline June & $5.6 \times 10^{-17} \pm 6 \%$ & $3.0 \times 10^{-18} \pm 10 \%$ & $5.4 \times 10^{-2}$ & $7.4 \times 10^{-10} \pm 2 \%$ & $1.7 \times 10^{-7}=4 \%$ & $4.4 \times 10^{-3}$ \\
\hline July & $1.9 \times 10^{-17} \pm 8 \%$ & $2.4 \times 10^{-18} \pm 12 \%$ & $1.3 \times 10^{-1}$ & $6.5 \times 10^{-10} \pm 3 \%$ & $1.1 \times 10^{-7} \pm 4 \sigma_{0}$ & $5.9 \times 10^{-3}$ \\
\hline Alig. & $6.7 \times 10^{-18} \pm 18 \%$ & $5.7 \times 10^{-19} \pm 52 \%$ & $8.5 \times 10^{-2}$ & $1.2 \times 10^{-n} \pm 2 \%$ & $3.3 \times 10^{-7} \pm 4_{i q}^{q}$ & $3.6 \times 10^{-3}$ \\
\hline Sepi. & $9.0 \times 10^{-18} \ddagger 8 \%$ & $8.4 \times 10^{-19} \pm 16 \%$ & $9.3 \times 10^{-2}$ & $1.3 \times 10^{-9} \pm 2^{\sigma \%}$ & $3.7 \times 10^{-7} \pm 4 \%$ & $3.5 \times 10^{-3}$ \\
\hline Oct. & $1.3 \times 10^{-17}+11 \%$ & $1.2 \times 10^{-18} \pm 11 \%$ & $9.2 \times 10^{-2}$ & $9.4 \times 10^{-10} \pm 2 \%$ & $2.2 \times 10^{-7} \pm 3$ & $4.3 \times 10^{-3}$ \\
\hline Nov. & $6.5 \times 10^{-i 8} \pm 8 \%$ & $4.0 \times 10^{-19} \pm 19 \%$ & $6.1 \times 10^{-2}$ & $4.5 \times 10^{-10} \pm 4$ & $1.5 \times 10^{-7} \pm 5 \%$ & $3.0 \times 10^{-3}$ \\
\hline Dec, & $7.1 \times 10^{-18} \pm 8 \%$ & $5.5 \times 10^{-19} \pm 17 \%$ & $7.7 \times 10^{-2}$ & $1.8 \times 10^{-10} \pm 3 \%$ & $6.2 \times 10^{-8} \pm 5 \%$ & $2.9 \times 10^{-3}$ \\
\hline Annual av & $1.7 \times 10^{-17}$ & $1.6 \times 10^{-18}$ & $9.4 \times 10^{-2}$ & $7.8 \times 10^{-10}$ & $2.4 \times 10^{-7}$ & \\
\hline $\mathrm{nCG}^{\mathrm{a}}$ & $1.0 \times 10^{-12}$ & $1.0 \times 10^{-12}$ & & $1.9 \times 10^{-3}$ & $1.5 \times 10^{-2}$ & \\
\hline$\%$ RCG & $1.7 \times 10^{-3}$ & $1.6 \times 10^{-4}$ & & $4.1 \times 10^{-5}$ & $1.5 \times 10^{-3}$ & \\
\hline
\end{tabular}

Assumes Pu to be in an insoluble form.

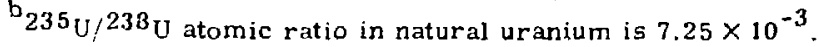


Table 20. Concentrations of uranium in Site 300 soils $(\mu \mathrm{g} / \mathrm{g}$ of dry soill.

\begin{tabular}{|c|c|c|c|c|c|c|c|c|c|}
\hline $\begin{array}{c}\text { Sampling } \\
\text { luca- } \\
\text { tion } \\
\end{array}$ & $\begin{array}{c}\text { Depth } \\
(\mathrm{cm})\end{array}$ & ${ }^{234} \mathrm{U}$ & $235_{U}$ & ${ }^{236} \mathrm{u}$ & $238 \mathrm{U}$ & ${ }^{235} \mathrm{U}^{23 \mathrm{~B}_{\mathrm{u}}} \mathrm{u}^{\mathrm{b}}$ & $\begin{array}{c}\begin{array}{c}\text { Total } \\
\text { uranium }\end{array} \\
\end{array}$ & $\begin{array}{c}\text { Total } \\
\text { natural } \\
\text { uranium } \\
\end{array}$ & $\begin{array}{c}\text { Total } \\
\text { depleted } \\
\text { uranium } \\
\end{array}$ \\
\hline$S-321$ & $0-1$ & $1.08 \times 10^{-4} \pm 8 \%$ & $1.56 \times 10^{-2} \pm 2 \%$ & $6.26 \times 10^{-6} \pm 50^{\circ}$ & $2.22 \pm 2 \%$ & $7.0 \times 10^{-3}$ & $2.24 \pm 2 \%$ & $2.18 \pm 3^{\circ}$ & $6.00 \times 10^{-2}: 100^{n}$ \\
\hline$S-322$ & $0-1$ & $8.00 \times 10^{-5} \pm 24 \%$ & $1.21 \times 10^{-2} \pm 3 \%$ & $3.15 \times 10^{-5} \pm 38 \sigma^{\circ}$ & $2.21 \pm 4 \%$ & $5.5 \times 10^{-3}$ & $2.22 \div 4 \%$ & $1.52 \pm 4^{-0}$ & $7.00 \times 10^{-1} \cdot 18^{n}$ \\
\hline$S-323$ & $0-1$ & $7.89 \times 10^{-5} \pm 8 \%$ & $1.07 \times 10^{-2} \pm 2 \%$ & $1.08 \times 10^{-5} \pm 20 \%$ & $1.64 \pm 3 \%$ & $6.5 \times 10^{-3}$ & $1.65 \pm 3 \sigma$ & $1.46 \pm 5 \%$ & $1.85 \times 10^{-1} \div 35 \%$ \\
\hline$S-324$ & $0-1$ & $7.65 \times 10^{-5} \pm 9 \%$ & $1.22 \times 10^{-2} \pm 2 \%$ & $2.16 \times 10^{-5} \pm 15 \%$ & $2.14 \pm 3_{n}^{\sigma}$ & $5.7 \times 10^{-3}$ & $2.15 \div 3 \%$ & $1.58 \pm 6 \%$ & $5.73 \times 10^{-1}: 1:$ \\
\hline$S-325$ & $0-1$ & $9.55 \times 10^{-5} \pm 8 \%$ & $1.41 \times 10^{-2} \pm 2 \%$ & $1.26 \times 10^{-5} \pm 20 \%$ & $2.27 \pm 3 \%$ & $6.2 \times 10^{-3}$ & $2.28 \pm 3 \%$ & $1.88+6^{\prime \prime \prime}$ & $4.03 \times 10^{-1} \div 26$ \\
\hline$S-326$ & $0-1$ & $6.43 \times 10^{-5} \pm 10 \%$ & $1.11 \times 10^{-2}+2 \%$ & $3.6 \times 10^{-5} \pm 11 \%$ & $2.39 \pm 4 \%$ & $4.8 \times 10^{-3}$ & $2.40 \pm 4 \%$ & $1.28 \div 6 \%$ & $1.13+110$ \\
\hline $5-327$ & $0-1$ & $1.13 \times 10^{-3} \pm 5 \%$ & $2.96 \times 10^{-1} \pm 2 \%$ & $4.0 \times 10^{-3}+3 \%$ & $150 \pm 5 \%$ & $2.0 \times 10^{-3}$ & $150 \pm 5 \%$ & $5.47+97$ & $1.45 \times 10^{-2} \div 75$ \\
\hline$S-328$ & $0-1$ & $1.44 \times 10^{-4} \pm 6 \%$ & $3.12 \times 10^{-2} \pm 2 \%$ & $3.45 \times 10^{-4} \pm 4 \%$ & $11.9+5 \%$ & $2.6 \times 10^{-3}$ & $11.9+5 \%$ & $1.89 \pm 8 \sigma_{i t}^{\sigma_{i}}$ & $1.00 \times 10^{1}+8^{2}$ \\
\hline$S-329$ & $0-1$ & $8.73 \times 10^{-5} \pm 8 \%$ & $1.46 \times 10^{-2} \pm 2 \%$ & $3.83 \times 10^{-5} \pm 11 \%$ & $3.07 \pm 4 \%$ & $4.8 \times 10^{-3}$ & $3.08+4 \%$ & $1.70 \pm 6 \%$ & $1.38+11 \%$ \\
\hline $5-330$ & $0-1$ & $9.56 \times 10^{-5}+8 \%$ & $1.40 \times 10^{-2} \pm 2 \%$ & $1.01 \times 10^{-5}+11 \%$ & $2.19 \pm 3^{\circ}$ & $6.4 \times 10^{-3}$ & $2.20 \pm 3 \%$ & $1.90 \div 6^{z_{n}^{\prime}}$ & $3.04 \times 10^{-1}: 347$ \\
\hline$S-331$ & $0-1$ & $1.34 \times 10^{-4} \pm 7 \%$ & $2.03 \times 10^{-2} \pm 2 \%$ & $1.94 \times 10^{-5} \pm 15^{\%}$ & $3.28 \pm 3 \%$ & $6.2 \times 10^{-3}$ & $3.30 \pm 3 \%$ & $2.71 \pm 6 \%$ & $5.85 \times 10^{-1}=26^{n}$ \\
\hline
\end{tabular}

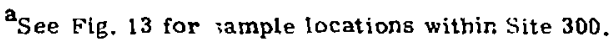

${ }^{6} 235_{U} / 238 \mathrm{U}$ atom c ratio of natural uranium is $7.25 \times 10^{-3}$. 
Table 21. Gross beta activity in Site 300 water samples $(\mu \mathrm{Cl} / \mathrm{ml})$.

\begin{tabular}{|c|c|c|c|c|c|c|c|c|c|c|}
\hline \multirow[b]{2}{*}{ Location } & \multirow{2}{*}{$\begin{array}{c}\text { No. of } \\
\text { samples }\end{array}$} & \multicolumn{3}{|c|}{$J$ anuary - June } & \multirow{2}{*}{$\begin{array}{c}\text { No. of } \\
\text { samples }\end{array}$} & \multicolumn{3}{|c|}{ July-December } & \multirow{2}{*}{$\begin{array}{c}\text { Annua! } \\
\text { average }\end{array}$} & \multirow[b]{2}{*}{$\mathrm{RCG}^{\mathrm{a}}$} \\
\hline & & Maximum & Minimum & Average & & Maximum & Minimum & Averige & & \\
\hline 1 & 6 & $6.0 \times 10^{-9} \pm 32 \%$ & $1.6 \times 10^{-9} \pm 74^{\sigma}$ & $3.9 \times 10^{-9}$ & 6 & $4.9 \times 10^{-9}+30 \%$ & $1.7 \times 10^{-9} \pm 72 \%$ & $3.7 \times 10^{-9}$ & $3.8 \times 10^{-9}$ & 13 \\
\hline 2 & 6 & $7.5 \times 10^{-9} \pm 24 \%$ & $5.1 \times 10^{-9} \pm 30 \%$ & $6.3 \times 10^{-9}$ & 6 & $6.9 \times 10^{-9}=26 \%$ & $2.6 \times 10^{-9} \pm 47 \%$ & $5.2 \times 10^{-9}$ & $5.8 \times 10^{-9}$ & 19 \\
\hline 3 & 6 & $7.1 \times 10^{-9} \pm 23 \%$ & $5.4 \times 10^{-9} \pm 315$ & $6.2 \times 10^{-9}$ & 6 & $7.5 \times 10^{-9} \pm 24 \%$ & $2.8 \times 10^{-9} \pm 40 \%$ & $4.6 \times 10^{-9}$ & $5.4 \times 10^{-9}$ & 18 \\
\hline 4 & 6 & $7.6 \times 10^{-9} \pm 24 \%$ & $3.0 \times 10^{-9}+48 \%$ & $4.6 \times 10^{-9}$ & 6 & $6.4 \times 10^{-9} \pm 26^{c}$ & $3.3 \times 10^{-9} \pm 30^{\sigma}$ & $4.8 \times 10^{-9}$ & $4.7 \times 10^{-8}$ & 16 \\
\hline 5 & 6 & $6.0 \times 10^{-9} \pm 27 \%$ & $4.2 \times 10^{-9} \pm 37 \%$ & $4.9 \times 10^{-9}$ & 6 & $8.7 \times 10^{-9} \pm 23 \%$ & $3.7 \times 10^{-9}=30 \%$ & $5.9 \times 10^{-y}$ & $5.4 \times 10^{-9}$ & 18 \\
\hline 6 & 6 & $5.4 \times 10^{-9} \pm 31 \%$ & $3.4 \times 10^{-9} \pm 41 \%$ & $4.4 \times 10^{-9}$ & 5 & $6.1 \times 10^{-9} \pm 27$ & $3.0 \times 10^{-9} \div 46 \%$ & $4.3 \times 10^{-9}$ & $4.4 \times 10^{-19}$ & 15 \\
\hline 7 & 6 & $6.8 \times 10^{-9} \pm 24 \%$ & $4.7 \times 10^{-9} \pm 34 \%$ & $6.0 \times 10^{-9}$ & 6 & $6.0 \times 10^{-9} \pm 2 \sigma^{t}$ & $3.7 \times 10^{-9} \pm 39 \%$ & $4.3 \times 10^{-5}$ & $5.2 \times 10^{-5}$ & 17 \\
\hline 11 & 6 & $1.1 \times 10^{-8} \pm 18^{\sigma} \%$ & $4.6 \times 10^{-9} \pm 32 \%$ & $8.2 \times 10^{-9}$ & 6 & $8.3 \times 10^{-9} \pm 215$ & $4.9 \times 10^{-9} \pm 32^{\sigma}$ & $6.6 \times 10^{-9}$ & $8.4 \times 10^{-9}$ & 27 \\
\hline 14 & 6 & $5.9 \times 10^{-9} \pm 27 \%$ & $1.8 \times 10^{-9} \pm 62 \%$ & $4.0 \times 10^{-9}$ & 2 & $6.4 \times 10^{-9} \pm 26 \%$ & $4.6 \times 10^{-9} \pm 33^{\sigma}$ & $5.5 \times 10^{-9}$ & $4.8 \times 10^{-9}$ & 16 \\
\hline 20 & $-{ }^{b}$ & & & & 2 & $9.7 \times 10^{-9} \pm 20 \%$ & $1.7 \times 10^{-9} \pm 100 \%$ & $5.7 \times 10^{-9}$ & & \\
\hline 21 & 3 & $1.1 \times 10^{-8} \pm 18 \%$ & $9.6 \times 10^{-9} \div 19 \%$ & $1.0 \quad 10^{-8}$ & 4 & $8.4 \times 10^{-9} \pm 21 \%$ & $3.9 \times 10^{-9} \pm 38 \%$ & $7.0 \times 10^{-9}$ & $8.5 \times 10^{-9}$ & 28 \\
\hline 25 & 4 & $3.9 \times 10^{-9} \pm 34 \%$ & $1.6 \times 10^{-9} \neq 100 \%$ & $2.3 \times 10^{-9}$ & $-b$ & & & & & \\
\hline 26 & 3 & $9.2 \times 10^{-9} \pm 18^{\sigma}$ & $5.3 \times 10^{-9} \pm 27^{c}$ & $7.0 \times 10^{-9}$ & -0 & & & & & \\
\hline
\end{tabular}


Table 22. Tritium in water samples from Site 3001973.

\begin{tabular}{|c|c|c|c|c|c|c|c|c|c|c|c|}
\hline \multirow[b]{2}{*}{ Location } & \multirow{2}{*}{$\begin{array}{c}\text { No. of } \\
\text { samples }\end{array}$} & \multicolumn{3}{|c|}{ January-June } & \multirow{2}{*}{$\begin{array}{c}\text { No of } \\
\text { samples }\end{array}$} & \multicolumn{3}{|c|}{ July-December } & \multirow{2}{*}{$\begin{array}{l}\text { Innual } \\
\text { average }\end{array}$} & \multirow[b]{2}{*}{$\therefore=\mathrm{HCG}^{\mathrm{a}}$} & \multirow{2}{*}{$\begin{array}{l}\text { Culculated } \\
\text { drnisal } \\
\text { adult } \\
\text { whole } \\
\text { boxly dose } \\
\text { 'mrem) }\end{array}$} \\
\hline & & Maximum & Minimum & Average & & Maximum & Minimum & Average & & & \\
\hline 1 & 0 & $2.1 \times 10^{-8} \pm 30.0 \%$ & $6.2 \times 10^{-9} \pm 78.9 \%$ & $1.1 \times 10^{-8}$ & 5 & $2.9 \times 10^{-5} \neq 25.3 \%$ & $4.8 \times 10^{-10}=50.1 \%$ & $1.3 \times 10^{-8}$ & $1.2 \times 10^{-8}$ & $4.0 \times 10^{-4}$ & $4.8 \times 10^{-3}$ \\
\hline 2 . & 6 & $8.0 \times 10^{-8} \pm 10.2 \%$ & $7.1 \times 10^{-9} \pm 86.4 \%$ & $2.6 \times 10^{-3}$ & 5 & $3.5 \times 10^{-8} \pm 22.1 \%$ & $1.4 \times 10^{-8} \neq 63.6 \%$ & $2.1 \times 10^{-8}$ & $2.4 \times 10^{-8}$ & $8.0 \times 10^{-4}$ & $9.5 \times 10^{-3}$ \\
\hline 3 & 0 & $5.2 \times 10^{-8} \pm 12.7 \%$ & $4.9 \times 10^{-9}+100.0 \%$ & $2.0 \times 10^{-8}$ & s & $1.8 \times 10^{-8} \pm 44.8 \%$ & $" .1 \times 10^{-9} \geq 100.0 \%$ & $1.1 \times 10^{-8}$ & $1.5 \times 10^{-8}$ & $5.3 \times 10^{-4}$ & $6.4 \times 10^{-3}$ \\
\hline 4 & 6 & $8.5 \times 10^{-8} \pm 9.7 \%$ & $5.8 \times 10^{-9} \neq 100.0 \%$ & $3.0 \times 10^{-8}$ & 5 & $7.1 \times 10^{-8}=10.8 \%$ & $1.2 \times 10^{-8} \pm 62.2 \%$ & $2.8 \times 10^{-8}$ & $2.9 \times 10^{-8}$ & $0.7 \times 10^{-4}$ & $3.2 \times 10^{-2}$ \\
\hline 5 & 0 & $8.3 \times 10^{-8} \pm 8.3 \%$ & $1.4 \times 10^{-8} \pm 45.5 \%$ & $4.2 \times 10^{-8}$ & 5 & $4.9 \times 10^{-8} \div 14.4 \%$ & $2.0 \times 10^{-8} \div 38.8 \%$ & $3.6 \times 10^{-8}$ & $3.9 \times 10^{-8}$ & $1.3 \times 10^{-3}$ & $1.5 \times 10^{-2}$ \\
\hline 6 & 6 & $2.5 \times 10^{-8}+22.4 \%$ & $5.5 \times 10^{-9} \pm 100.0 \%$ & $1.2 \times 10^{-8}$ & 5 & $2.9 \times 10^{-8} \pm 25.8 \%$ & $6.2 \times 10^{-9}=100.0 \%$ & $1.7 \times 10^{-3}$ & $1.4 \times 10^{-8}$ & $4.7 \times 10^{-4}$ & $5.6 \times 10^{-3}$ \\
\hline 7 & 6 & $9.1 \times 10^{-8} \pm 8.5 \%$ & $2.7 \times 10^{-8} \pm 25.9 \%$ & $4.9 \times 10^{-8}$ & 5 & $5.0 \times 10^{-8} \pm \mathrm{i} 7.4 \%$ & $6.8 \times 10^{-9} \neq 100.0 \%$ & $2.4 \times 10^{-8}$ & $3.8 \times 10^{-8}$ & $1.3 \times 10^{-3}$ & $1.5 \times 10^{-2}$ \\
\hline 11 & 6 & $1.3 \times 10^{-7}+6.5 \%$ & $6.5 \times 10^{-9} \neq 95.0 \%$ & $5.3 \times 10^{-8}$ & 5 & $2.3 \times 10^{-8} \div 31.4 \%$ & $7.8 \times 10^{-9} \div 100.07$ & $1.4 \times 10^{-8}$ & $3.6 \times 10^{-8}$ & $1.2 \times 10^{-3}$ & $1.4 \times 10^{-2}$ \\
\hline 14 & 5 & $1.6 \times 10^{-7} \neq 7.0^{\sigma}$ & $1.1 \times 10^{-7} \pm 7.2 \%$ & $1.3 \times 10^{-7}$ & 2 & $1.5 \times 10^{-7} \pm 7.37$ & $1.4 \times 10^{-7} \neq 7.2 \%$ & $1.5 \times 10^{-7}$ & $1.4 \times 10^{-7}$ & $4.3 \times 10^{-4}$ & $5.2 \times 10^{-2}$ \\
\hline 20 & 4 & $1.2 \times 10^{-7} \pm 6.6 \%$ & $7.8 \times 10^{-8} \pm 10.0 \%$ & $1.0 \times 10^{-7}$ & 1 & $6.1 \times 10^{-8}+13.0$. & $6.1 \times 10^{-8} \pm 13.0 \%$ & $0.1 \times 10^{-8}$ & $9.4 \times 10^{-8}$ & $3.1 \times 10^{-3}$ & $3.8 \times 10^{-2}$ \\
\hline 21 & 3 & $6.4 \times 10^{-8} \pm 11.7 \%$ & $3.3 \times 10^{-8} \pm 18.8 \%$ & $4.4 \times 10^{-8}$ & 3 & $5.5 \times 10^{-8} \pm 14.15$ & $4.1 \times 10^{-8} \pm 17.3 \%$ & $4.6 \times 10^{-8}$ & $4.5 \times 10^{-8}$ & $1.5 \times 10^{-3}$ & $1.8 \times 10^{-2}$ \\
\hline $25^{\circ}$ & 4 & B. $5 \times 10^{-4} \pm 2.0 \%$ & $5.2 \times 10^{-4}+2.05$ & $6.9 \times 10^{-4}$ & 0 & & & & & & \\
\hline 26 & 2 & $1.8 \times 10^{-8} \pm 38.2 \%$ & $1.0 \times 10^{-8} \pm 6^{7} .77^{\mathrm{e}}$ & $1.4 \times 10^{-8}$ & $\underline{0}$ & & & & $1.4 \times 10^{-8}$ & $4.6 \times 10^{-4}$ & $5.6 \times 10^{-2}$ \\
\hline
\end{tabular}


$\because$ able 23. letivities of various radionuclides in Site 300 vegetation samples $(\mu \mathrm{Ci} / \mathrm{g})$.

\begin{tabular}{cccccc}
\hline Radionuclide & Maximum & Minimum & $\begin{array}{c}\text { Annual } \\
\text { average }\end{array}$ & $\begin{array}{c}\text { Calculated annual } \\
\text { dose via direct } \\
\text { ingestion } \\
\text { (mrem) }\end{array}$ & Critical organ \\
\hline${ }^{7} \mathrm{Be}$ & $6.5 \times 10^{-6}$ & $3.3 \times 10^{-7}$ & $2.2 \times 10^{-6}$ & 0.1 & Lower large intestine \\
${ }^{40} \mathrm{~K}$ & $.0 \times 10^{-5}$ & $1.2 \times 10^{-5}$ & $2.3 \times 10^{-5}$ & 33.5 & Whole body \\
${ }^{95} \mathrm{Zr}$ & $2.4 \times 10^{-7}$ & $2.9 \times 10^{-8}$ & $1.3 \times 10^{-7}$ & 0.3 & Lover large intestine \\
${ }^{103} \mathrm{Ru}$ & $3.0 \times 10^{-7}$ & $2.3 \times 10^{-8}$ & $9.8 \times 10^{-8}$ & 0.1 & Lower large intestine \\
${ }^{137} \mathrm{Cs}$ & $1.5 \times 10^{-7}$ & $1.7 \times 10^{-8}$ & $5.5 \times 10^{-8}$ & 0.1 & Whole body \\
${ }^{141} \mathrm{Ce}$ & $1.1 \times 10^{-7}$ & $1.4 \times 10^{-8}$ & $5.0 \times 10^{-8}$ & 0.05 & Lower large intestine \\
${ }^{144} \mathrm{Ce}$ & $3.3 \times 10^{-7}$ & $2.4 \times 10^{-8}$ & $1.5 \times 10^{-7}$ & 1.2 & Lnwer large intestine
\end{tabular}


Table 24. Tritium in vegetation samples from site $3001973(\mu \mathrm{Ci} / g)$.

\begin{tabular}{|c|c|c|c|c|c|c|c|c|c|c|}
\hline Location & $\begin{array}{c}\text { No, of } \\
\text { samples }\end{array}$ & Maximum & Minimum & Average & $\begin{array}{r}\text { No. of } \\
\text { samples }\end{array}$ & Mix. $\mathrm{n}_{\mathrm{n}}$ & Minimum & Average & $\begin{array}{l}\text { Annual } \\
\text { average }\end{array}$ & $\begin{array}{c}\text { Cilculated } \\
\text { innual } \\
\text { adult } \\
\text { whole } \\
\text { body dose } \\
\text { (marem) }\end{array}$ \\
\hline 1 & 6 & $1.2 \times 10^{-6} \pm 76.5^{\%} \%$ & $1.4 \times 10^{-7} \pm 100.0 \%$ & $5.0 \times 10^{-7}$ & 6 & $1.9 \times 10^{-6} \pm 100.0 \%$ & $7.0 \times 10^{-8} \pm 100.0 \%$ & $6.0 \times 10^{-7}$ & $5.5 \times 10^{-7}$ & $8.3 \times 10^{-3}$ \\
\hline 2 & 6 & $1.2 \times 10^{-6} \pm 37.8 \%$ & $3.0 \times 10^{-7} \pm 100.0 \%$ & $7.1 \times 10^{-7}$ & 6 & $3.2 \times 10^{-6} \pm 17.6 \%$ & $2.1 \times 10^{-7} \pm 100.0 \%$ & $1.0 \times 10^{-6}$ & $8.7 \times 10^{-7}$ & $1.3 \times 10^{-2}$ \\
\hline 3 & 6 & $8.5 \times 10^{-7} \pm 63.9 \%$ & $2.6 \times 10^{-1} \pm 74.0 \%$ & $5.1 \times 10^{-7}$ & 6 & $2.4 \times 10^{-6} \pm 35.5 \%$ & $2.6 \times 10^{-7} \pm 87.9 \%$ & $3.4 \times 10^{-7}$ & $6.8 \times 10^{-7}$ & $1.0 \times 10^{-2}$ \\
\hline 4 & 6 & $1.3 \times 10^{-6} \pm 37.2 \%$ & $4.7 \times 10^{-7} \pm 100.0 \%$ & $7.3 \times 10^{-7}$ & 6 & $1.7 \times 10^{-6} \pm 29.5 \%$ & $2.8 \times 10^{-7} \pm 87.9 \%$ & $7.7 \times 10^{-7}$ & $7.5 \times 10^{-7}$ & $1.1 \times 10^{-2}$ \\
\hline 5 & $i$ & $1.1 \times 10^{-6} \pm 71.1 \%$ & $4.0 \times 10^{-7} \pm 100.0 \%$ & $7.7 \times 10^{-7}$ & 6 & $1.0 \times 10^{-6} \pm 38.1 \%$ & $5.3 \times 10^{-8} \pm 100.0 \%$ & $4.8 \times 10^{-7}$ & $6.1 \times 10^{-7}$ & $0.2 \times 10^{-3}$ \\
\hline 6 & $\mathbf{5}$ & $1.6 \times 10^{-4} \pm 31.7 \%$ & $4.2 \times 10^{-6} \pm 15.2 \%$ & $4.2 \times 10^{-5}$ & a & $8.4 \times 10^{-5} \pm 1.4 \%$ & $3.3 \times 10^{-6} \pm 7.0 \%$ & $4.3 \times 10^{-5}$ & $4.3 \times 10^{-5}$ & $6.5 \times 10^{-1}$ \\
\hline 7 & 6 & $9.1 \times 10^{-7} \pm 42.8 \%$ & $2.4 \times 10^{-7} \pm 99.3 \%$ & $5.3 \times 10^{-7}$ & 6 & $1.3 \times 10^{-5} \pm 9.1 \%$ & $2.3 \times 10^{-7} \pm 100.0 \%$ & $2.6 \times 10^{-6}$ & $1.5 \times 10^{-6}$ & $2.3 \times 10^{-2}$ \\
\hline 8 & 5 & $6.3 \times 10^{-7} \pm 89.1 \%$ & $2.3 \times 10^{-7}+100.0 \%$ & $4.2 \times 10^{-7}$ & 5 & $9.6 \times 10^{-6} \pm 11.5 \%$ & $1.8 \times 10^{-7} \pm 100.07$ & $2.2 \times 10^{-6}$ & $1.3 \times 10^{-6}$ & $2.0 \times 10^{-2}$ \\
\hline ? & 5 & $2.3 \times 10^{-6} \pm 18.1 \%$ & $4.0 \times 10^{-7} \pm 100.0 \%$ & $1.4 \times 10^{-6}$ & 6 & $1.9 \times 10^{-6} \pm 14.4 \%$ & $6.4 \times 10^{-7} \pm 28.7 \%$ & $1.1 \times 10^{-6}$ & $1.2 \times 10^{-6}$ & $1.8 \times 10^{-2}$ \\
\hline 10 & 6 & $1.1 \times 10^{-6} \pm 30.2 \%$ & $2.6 \times 10^{-7} \pm 83.5 \%$ & $6.6 \times 10^{-7}$ & 6 & $2.1 \times 10^{-6} \pm 33.4 \%$ & $1.4 \times 10^{-7} \pm 100.0 \%$ & $6.5 \times 10^{-7}$ & $6.6 \times 10^{-7}$ & $1.0 \times 10^{-2}$ \\
\hline 11 & 6 & $8.9 \times 10^{-7} \pm 70.4 \%$ & $3.6 \times 10^{-7} \pm 100.0 \%$ & $6.8 \times 10^{-7}$ & 6 & $3.2 \times 10^{-6} \pm 25.5 \%$ & $1.5 \times 10^{-7} \pm 100.0 \%$ & $1.3 \times 10^{-6}$ & $9.9 \times 10^{-7}$ & $1.5 \times 10^{-2}$ \\
\hline 12 & 1 & $1.5 \times 10^{-6} \pm 29.2 \%$ & $1.5 \times 10^{-6} \pm 29.2 \%$ & $1.5 \times 10^{-6}$ & 6 & $1.2 \times 10^{-6} \pm 61.9 \%$ & $1.4 \times 10^{-7} \times 63.8 \%$ & $5.0 \times 10^{-7}$ & $6.5 \times 10^{-7}$ & $9.8 \times 10^{-3}$ \\
\hline 13 & 5 & $2.7 \times 10^{-4} \pm 77.6 \%$ & $7.9 \times 10^{-6} \pm 4.5 \%$ & $7.4 \times 10^{-5}$ & 6 & $2.8 \times 10^{-5} \pm 2.7 \%$ & $2.8 \times 10^{-7} \pm 100.00$ & $8.4 \times 10^{-6}$ & $3.8 \times 10^{-5}$ & $5.7 \times 10^{-1}$ \\
\hline
\end{tabular}


Table 25. Radionuclides observed in milk during $1973(\mu \mathrm{Ci} / \mathrm{ml})$.

\begin{tabular}{ccccccc} 
Radio- & $\begin{array}{c}\text { No. of } \\
\text { nuclide samples }\end{array}$ & Maximum & Minimum & Average & \multicolumn{3}{c}{$\begin{array}{c}\text { Calculated } \\
\text { annual } \\
\text { adult } \\
\text { radiation } \\
\text { dose } \\
\text { (mrem) }\end{array}$} & $\begin{array}{c}\text { Critical } \\
\text { organ }\end{array}$ \\
\hline${ }^{137} \mathrm{Cs}$ & 12 & $2.4 \times 10^{-9} \pm 35 \%$ & $1.1 \times 10^{-9} \pm 62 \%$ & $1.6 \times 10^{-9}$ & $9.5 \times 10^{-3}$ & Whole body \\
${ }^{3}{ }_{\mathrm{H}}$ & 12 & $2.5 \times 10^{-7} \pm 54 \%$ & $1.1 \times 10^{-7} \pm 100 \%$ & $1.7 \times 10^{-7}$ & $5.2 \times 10^{-4}$ & Whole body \\
${ }^{40} \mathrm{~K}$ & 12 & $1.3 \times 10^{-6} \pm 5 \%$ & $1.2 \times 10^{-6} \pm 5 \%$ & $1.3 \times 10^{-6} 5.8$ & Whole body \\
\hline
\end{tabular}




\section{References}

1. P. H. Gudiksen, C. L. Lindeken, C. Gatrous1s, and L. R. Anspaugh, Environmental Levels of Radioactivity in the Vicinity of the Law rence Livermore Laboratory January Through December 1971, Lawrence Livermore Laboratory, Rept. UCRL-51242 (1972).

2. P. H. Gudiksen, C. L. Lindeken, J.W. Meadows, and K. O. Hamby, Environmental Levels of Radioactivity in the Vicinity of the Lawrence Livermore Laboratory 1972 Annual Report, Lawrence Livermore Laboratory, Rept. UCRL-51333 (1973).

3. Preliminary Safety Analysis Report (PSAR) for Building 332-Increment III, Lawrence Livermore Laboratory, Rept. UCRL-5.1436 (Draft Revision 1, January 18, 1974).

4. E. P. Hardy and P. W. Krey, "Determining the Accumulated Deposit of Radionuclides by Soil Sampling and Analysis." Proceedings of the Plutonium Symposium held at LASL August 4-5, 1971, Los Alamos Scientific Laboratory Rept. L (1971).

5. H. A. Wollenberg, H. W. Patterson, A. R. Smith, and L. D. Stephen, "Natural and Fallout Acitivity in the San Francisco Bay Area," Health Physics 17 (1969).

6. L. R. Anspaugh, J. J. Koranda, W. L. Robison, and J. R. Martin, The Dose to Man Via Food Chain Transfer Resulting from Exposure to Tritiated Water Vapor, Lawrence Livermore Laboratory, Rept. UCRL-73195 (1971).

7. Agriculture Statistics, 19E3, United States Department of Agriculture, U.S. Government Printing Office, Washington, D. C. (1969).

8. Y.C. Ng, C. Ann Burton, S. E. Thompson, R. K. Tandy, H. K. Kretner, and M.W. Pratt, Prediction of the Dosage to Man from the Fallout of Nuclear Devices, IV: Handbook for Estimating the Internal Dose from Radionuclides Released to the Biosphere, Lawrence Livermore Laboratory, Rept. UCRL-50163 (1968).

9. W. M. Lowder and H. L. Beck, "Cosmic-ray lonization in the Lower Atmosphere," J. Geo. Phys. Res. 71, 4611 (1966). 


\section{Appendix - Environmental Activity Guide Levels}

The Standards for Radiation Protection (AEC Manual Chapter 0524, issued 11/8/68) state that if there is a mixture in air and water of radionu:lides whose identity and concentrati- .s are unknown, the average activity should not exceed the following values:
1. Air (control]ed area)
$6 \times 10^{-12} \mu \mathrm{Cl} / \mathrm{ml}$
2. Air (uncontrolled are
$2 \times 10^{i 4} \mu \mathrm{Ci} / \mathrm{ml}$
3. Water (controlled area)
$4 \times 10^{-7} \mu \mathrm{Ci} / \mathrm{ml}$
4. Water (uncontrolled area)
$3 \times 10^{-8} \mu \mathrm{Ci} / \mathrm{ml}$

If it is known that alpha emitters and 227 Ac are not present, the following guide values may be used to determine the permissible average activity:
5. Air (controlled area)
$3 \times 10^{-11} \mu \mathrm{Ci} / \mathrm{ml}$
6. Air (uncontrolled area)
$1 \times 10^{-12} \mu \mathrm{Ci} \cdot \mathrm{ml}$

If it is known that ${ }^{129} 1,{ }^{226} \mathrm{Ra}$, and ${ }^{228} \mathrm{Ra}$ are not present, the following values may be used:
7. Water (controlled area)
8. Water (uncuntrolled area)

$3 \times 10^{-6} \mu \mathrm{Ci} / \mathrm{ml}$
$1 \times 10^{-7} \mu \mathrm{Ci} / \mathrm{ml}$

The air and water samples are subjected to gross alpha and gross beta measurements. The average annual alpha activities may not exceed those listed under points 1 through 4 above. Since the alpha emitters have been accounted for in the gross alpha measurements, and the assumption is made that ${ }^{129} \mathrm{I},{ }^{227} \mathrm{Ac},{ }^{226} \mathrm{Ra}$, and ${ }^{228} \mathrm{Ra}$ are not present in the samples, the annual average gross beta activities of the samples may not exceed the activities listed under points 5 through 8 above. The assumption that ${ }^{129} \mathrm{I},{ }^{227} \mathrm{Ac},{ }^{226} \mathrm{Ra}$, and ${ }^{228} \mathrm{Ra}$ are not present in air and $w$ ater samples is reasonable in view of the minute quantities of these radionuclides available at the Laboratory. AEC Manual Chapter 0524 also states that the average tritium activities in off-site water samples may not exceed $3 \times 10^{-3} \mu \mathrm{Ci} / \mathrm{ml}$.

Since analysis for ${ }^{129} \mathrm{I},{ }^{226} \mathrm{Ra}$, and ${ }^{228} \mathrm{Ra}$ activities is made on samples collected from the Laboratory's sewage effluent at the point of discharge into the Livermore city sanitary sewer system, the gross alpha and beta activities in the samples collected from the effluent discharged from the Livermore sewage treatment plant should not exceed the $1 \times 10^{-7} \mu \mathrm{Ci} / \mathrm{ml}$ listed under point 8 above.

The annual external whole body radiation dose to workers in controlled areas may not exceed 5 rem; while that to an individual in an uncontrolled area may not exceed 500 mrem. An average annual dose of $170 \mathrm{mrem}$ may not be exceeded for a grcup of individuals in an uncontrolled area. 